\title{
Modelo Autoregressivo Vetorial com Coeficientes Variantes no Tempo e Aplicações em RMf
}

\author{
João Ricardo Sato
}

TESE APRESENTADA

AO

INSTITUTO DE MATEMÁTICA E ESTATÍSTICA

DA

UNIVERSIDADE DE SÃO PAULO

PARA

OBTENÇÃO DO GRAU DE DOUTOR

EM

ESTATÍSTICA

Área de Concentração: Estatística

Orientador: Prof. Dr. Pedro Alberto Morettin

Durante o desenvolvimento deste trabalho, o autor recebeu apoio financeiro do $\mathrm{CNPq}$ 


\section{Modelo Autoregressivo Vetorial com Coeficientes Variantes no Tempo e Aplicações em RMf}

Este exemplar corresponde à redação final da dissertação devidamente corrigida

e apresentada por João Ricardo Sato e aprovada pela comissão julgadora.

São Paulo, 29 de Março de 2007.

Banca examinadora:

- Prof. Dr. Pedro Alberto Morettin (orientador) (IME-USP)

- Prof. Dr. Chang Chiann (IME-USP)

- Prof. Dr. Denisard Cneio de Oliveira Alves (FEA-USP) 
Agradeço a Deus por todas as conquistas obtidas até hoje. Dedico este trabalho aos meus pais Liria e Orlando, por tudo o que me foi dado. Meus agradecimentos ao Prof. Pedro Alberto Morettin que desde a graduação me orienta e aconselha em todas as atividades acadêmicas. Ao Prof. Edson Amaro Jr. não só pela co-orientação e ajuda profissional, mas também pela amizade e exemplo. Meus sinceros votos de agradecimento ao Prof. Michael John Brammer, por todas as colaborações e conselhos, que não se restringem somente ao período de estágio em Londres.

Agradeço especialmente à Cristina pelo carinho e apoio em todos os momentos, aos meus irmãos Cristiane, Rafael, Paula, Priscila e a todos os familiares por estarem presentes em minha vida. Aos meus amigos Cinthia, D. Neusa, Daniel, Fujita, Gladys, Rogério, Janaína, Sergi, Christine, Sarah, Vincent, Márcio, Taty, Paulo, Ednei, Carlos, Paula Arantes, Lim, Gracinha, Maria Otaduy, Dra. Claudia, Fabio Barros, Cesário, Claudinei, Cacau, Anderson Winkler, Marcos Akiba, Mariana, Paty Lessa, Maria Ângela, Griselda, Luis Chaves, Cristofer, Fernanda, Suzan, Adriana, Seiko e a todos os demais que esqueci de citar.

Enfim, meus votos de agradecimento à Profa. Chang Chiann, ao Prof. Baccalá e ao Prof. Sameshima por todos os conselhos e sugestões. 


\begin{abstract}
Resumo
Os avanços nas técnicas de neuroimagem, principalmente com o desenvolvimento da ressonância magnética funcional (RMf), vêm possibilitando um melhor compreendimento dos processos e mecanismos cerebrais. Este trabalho tem como objetivo o desenvolvimento de um modelo de conectividade dinâmico entre diversas áreas cerebrais utilizando dados de RMf. A modelagem dinâmica do fluxo de informações é realizada com a estimação dos parâmetros de um modelo autoregressivo multivariado com coeficientes variandos no tempo, baseado na projeção de funções em bases de ondaletas. Dessa forma, um método para estimação e a derivação de suas propriedades assintóticas são apresentados. Diversos conjuntos de simulações computacionais são realizados visando a avaliação do desempenho do método proposto. Por fim, são apresentadas aplicações do modelo de conectividade variante no tempo em dados de ressonância magnética funcional.
\end{abstract}




\begin{abstract}
Advances in neuroimage technologies, mainly with the development of functional magnetic resonance imaging (fMRI), improve the comprehension of brain processes and mechanisms. The main goal of this work is the development of a time-varying connectivity model between many brain areas using fMRI datasets. The dynamic modelling of the information flow is related to the parameters estimation of a timevarying multivariate autoregressive process, based on functions projection in wavelet basis. We propose an estimation procedure and present its asymptotic properties. Computational simulations were performed focusing the evaluation of the proposed approach. Further, applications of these methodologies to real functional magnetic resonance datasets are presented.
\end{abstract}


Alguns jargões e abreviações:

BOLD: Blood Oxigenation Level Dependent. É o sinal medido em RMf.

Conectividade Efetiva: Influência de um módulo neuronal sobre outro.

Conectividade Funcional: Correlação na atividade de dois módulos neuronais.

DVAR: Modelo autoregressivo vetorial com coeficientes variando no tempo.

fatia: Imagem de uma seç̧ão cerebral.

GLM: Modelo linear geral.

HRF: Função de resposta hemodinâmica.

Mapa de Ativação: Imagem representando as áreas cerebrais ativadas durante uma tarefa específica.

Paradigma: Conjunto de atividades a ser executada por um indivíduo em um experimento de ressonância magnética funcional.

pixel: célula de uma matriz de brilhos, que de descreve uma imagem bidimensional.

RMf: ressonância magnética funcional.

ROI: região de interesse.

scan: aquisição de um volume.

sessão: conjunto de volumes de RMf adquiridos a cada vez que um indivíduo entra no scanner.

slice: vide fatia.

TR: intervalo de tempo entre a aquisição de dois volumes consecutivos.

VAR: Modelo autoregressivo vetorial.

volume: Conjunto de fatias, que representa o cérebro como um todo. voxel: pixel que compõe um volume cerebral. 


\section{Conteúdo}

Introdução

1 Ressonância Magnética Funcional 3

1.1 Ressonância Magnética Funcional . . . . . . . . . . . . . . . . . 3

1.2 Processamento de Imagens de RMf . . . . . . . . . . . . . . . . . . . . 8

1.2.1 Realinhamento . . . . . . . . . . . . . . . . . 9

1.2 .2 Normalização Espacial . . . . . . . . . . . . . . . . . . . . . . . 10

1.3 Mapeamento Cerebral . . . . . . . . . . . . . . . . . . . . . . 12

1.3.1 Experimentos e Paradigmas . . . . . . . . . . . . 13

1.3.2 Alguns Modelos . . . . . . . . . . . . . . . . . 14

2 Ondaletas $\quad 18$

2.1 Funções de Ondaletas . . . . . . . . . . . . . . . . . . . . . 18

2.1 .1 Introdução . . . . . . . . . . . . . . . . . . . . 18

2.1.2 Análise Multirresolução . . . . . . . . . . . . . . . . . . 22

2.1.3 A transformada de Ondaletas . . . . . . . . . . . . . . 24

2.2 Análise de Ondaletas . . . . . . . . . . . . . . . 25

2.2 .1 Limiarização . . . . . . . . . . . . . . . . . . . . . 25 
2.2.2 Ondaletas e Processos Estacionários . . . . . . . . . . . . . . . 27

2.2 .3 Espectro de Ondaletas . . . . . . . . . . . . . . . . 28

3 Modelo Autoregressivo Vetorial $\quad 30$

3.1 Modelo Autoregressivo Vetorial . . . . . . . . . . . . . . . . . . 30

3.1 .1 Estimação dos Parâmetros . . . . . . . . . . . . . . . . . . 31

3.1.2 Direcionalidade . . . . . . . . . . . . . . . . . 33

3.1.3 Testes de Hipóteses . . . . . . . . . . . . . . . . . . . 34

3.2 Conectividade Cerebral e Causalidade de Granger . . . . . . . . . . . . . . 35

4 Modelo Autoregressivo Vetorial Variante no Tempo 40

4.1 Modelo Autoregressivo Multivariado Dinâmico . . . . . . . . . . . . . . . . 40

4.1.1 Estimação dos Parâmetros . . . . . . . . . . . . . . . . . . . . 43

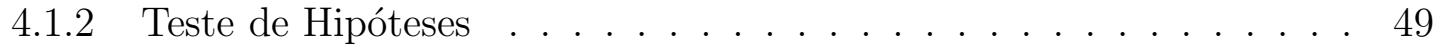

4.1 .3 Estimação da Matriz de Covariâncias . . . . . . . . . . . . . . . . . 50

4.2 Conectividade via DVAR . . . . . . . . . . . . . . . . . 52

4.3 Apêndice . . . . . . . . . . . . . . . . . . . . . 53

$\begin{array}{lll}5 & \text { Simulações } & 59\end{array}$

5.1 Simulação $1 \ldots \ldots \ldots \ldots$. . . . . . . . . . . . . . . . . . . . . 59

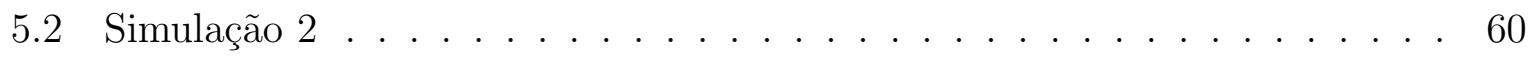

5.3 Simulação $3 \ldots \ldots$. . . . . . . . . . . . . . . . . . . . 73

5.4 Simulação $4 \ldots \ldots$. . . . . . . . . . . . . . . . . . . . . . . . . . . . . . . . . 88

5.5 Simulação $5 \ldots \ldots \ldots$. . . . . . . . . . . . . . . . . . . 88

5.6 Conclusões . . . . . . . . . . . . . . . . . . . . . . . . . . 91

6 Aplicações a Dados de RMf $\quad 94$ 
6.1 Experimento Motor . . . . . . . . . . . . . . . . . . . . . 94

6.2 Doença de Parkinson . . . . . . . . . . . . . . . . . . . 104

6.3 Experimento de Rotação Mental . . . . . . . . . . . . . . . . . . . . . . 110

7 Conclusão 


\section{Introdução}

Os avanços nas técnicas de neuroimagem vêm desempenhando um papel fundamental nos estudos de neurociências. Com o desenvolvimento da obtenção de imagens via ressonância magnética funcional (RMf) é possível identificar a atividade neuronal de cada área cerebral, através do monitoramento do fluxo e volume sangüineo, consumo de oxigênio e principalmente do sinal BOLD (blood oxigenation level dependent, Ogawa et al. (1990)). Por ser um método não invasivo e por sua alta resolução espacial comparado com outros métodos (e.g., PET, EEG, SPECT, etc), a RMf é a técnica de neuroimagem mais utilizada para o estudo de mecanismos cerebrais, e o seu uso continua crescendo rapidamente.

A maior parte dos estudos que envolvem RMf está concentrada na identificação de regiões cerebrais que são ativadas durante a execução de determinadas tarefas (teoria da especialização cortical (Friston et al., 2007)). Contudo, o entendimento da forma como as áreas cerebrais interagem entre si é fundamental para um melhor compreendimento da dinâmica dos circuitos neuronais (teoria da integração (Friston et al., 2007)). Assim, existe também o interesse na identificação e quantificação da conectividade entre as áreas cerebrais. Diversos métodos foram propostos para a avaliação da conectividade cerebral, tais como a análise de correlação do sinal BOLD de áreas distintas (Biswal et al., 1995), análise de componentes principais (PCA), análise de componentes independentes (ICA), modelagem via equações estruturais e mapas de caminhos (SEM, McIntosh (1999)), modelos causais dinâmicos (DCM, Friston et al. (2003)) e modelagem autoregressiva vetorial (VAR, Goebel et al. (2003)).

A suposição de estacionariedade do processo mental durante o período a ser analisado é assumida pela maioria dos métodos propostos. No entanto, é razoável esperar que tarefas 
cognitivas e emocionais, ao longo de sua execução, envolvam circuitos cerebrais distintos. Assim sendo, em experimentos de RMf onde o indivíduo executa determinada tarefa ou mais de uma tarefa específica, é natural esperar que o fluxo de informação cerebral seja alterado durante a realização do experimento. Além disso, não se sabe ao certo qual a velocidade de alteração entre um estado mental para outro, no entanto, é razoavel supor que esta variação não se dê instantaneamente.

O objetivo deste trabalho é introduzir uma nova técnica para a análise de conectividade cerebral, baseada no conceito de causalidade de Granger variando no tempo e decomposição de funções via ondaletas. Alguns estudos (Bullmore et al, 2001) mostram que a análise de ondaletas é útil para a modelagem dos dados de neuroimagem, principalmente devido a alta adaptabilidade e propriedades de multirresolução.

No primeiro capítulo, serão apresentados alguns conceitos básicos sobre as imagens obtidas através da ressonância magnética funcional. Serão apresentadas também metodologias para o pré-processamento dos dados e algumas técnicas para a análise estatística dos experimentos. Uma breve introdução às funções de ondaletas é apresentada no capítulo 2 .

No capítulo 3, apresentamos os modelos autoregressivos vetoriais e mostramos como utilizá-los para a modelagem e identificação do fluxo de informação entre áreas cerebrais. As principais metodologias desenvolvidas neste trabalho são apresentadas no capítulo 4, onde introduzimos o modelo autoregressivo vetorial com coeficientes variando no tempo, com propostas de um método para estimação, propriedades assintóticas e testes de hipóteses.

No capítulo 5, são apresentados diversos resultados obtidos através de simulações computacionais. Neste capítulo, as proposições e resultados teóricos obtidos no capítulo anterior são verificados empiricamente, em amostras finitas.

Por fim, aplicações do modelo proposto em três conjuntos de dados de RMf são ilustradas no capítulo 6. Apresenta-se uma aplicação do modelo autoregressivo vetorial com coeficientes variando no tempo em um experimento AB periódico com tarefa motora e resting, para dados envolvendo pacientes com mal de Parkinson comparativamente a voluntários normais e para tarefas cognitivas mais complexas envolvendo rotação mental . 


\section{Ressonância Magnética Funcional}

A ressonância magnética funcional é uma técnica de neuroimagem recente, a qual tem se mostrado extremamente útil para o entendimento das dinâmicas cerebrais. A técnica utiliza sistemas de ressonância magnética configurados de forma a identificar áreas que apresentam variações no fluxo sangüíneo e oxigenação. Neste capítulo apresentaremos alguns conceitos básicos para o entendimento da técnica e análises realizadas nos experimentos de RMf.

\subsection{Ressonância Magnética Funcional}

As metodologias para a identificação da atividade cerebral utilizando equipamentos de ressonância magnética vêm se desenvolvendo a uma velocidade surpreendente. Um método alternativo para a quantificação da atividade neuronal é a eletroencéfalografia (EEG). No entanto, embora tenha uma boa resolução temporal (alta taxa de amostragem em intervalos de tempo pequenos), a precisão na localização espacial é baixa e os sinais avaliados são praticamente pertencentes à área cortical (i.e., parte mais superficial do cérebro). Na década de 80, desenvolveu-se a técnica PET (Positron Emission Tomography), capaz de gerar imagens com boa localização espacial mas baixa resolução temporal (ordem de minutos). No entanto, estudos com PET envolvem a injeção de contrastes radioativos (método invasivo). A ressonância magnética funcional (RMf) vem se tornando uma ferramente essencial para o entendimento das dinâmicas cerebrais, tanto para processos que exigem 
o cumprimento de tarefas específicas, quanto para dados colhidos sem que o voluntário execute uma tarefa específicamente orientada (resting state). Os experimentos de RMf não envolvem a injeção de contrastes, são pouco-invasivos, e possuem maior resolução espacial comparado ao PET.

A técnica de ressonância magnética é baseada em três estágios (Amaro e Yamashita, 2001):

i) alinhamento: refere-se à propriedade magnética do núcleo de determinados tipos de átomos, que tendem a uma orientação paralela à um campo magnético. O núcleo de hidrogênio é o elemento utilizado para produzir imagens de seres biológicos, devido sua abundância (a maioria dos seres vivos tem hidrogênio em grande parte da sua composição). Para realizar o processo de orientação, freqüentemente utiliza-se um campo magnético de 1,5 Tesla (equivalente à 30 mil vezes o campo magnético terrestre). No entanto, alguns centros de neuroimagem utilizam equipamentos capazes de produzir campos de 3 Tesla, aumentanto a resolução espacial e razão sinal-ruido;

ii) excitação: o núcleo de cada elemento com número atomico ímpar tem uma "vibração" em uma freqüência proporcional ao campo magnético que está localizado. Para um campo de 1,5T, o hidrogênio tem uma freqüência de vibração de aproximadamente 63,8MHz. Assim, o aparelho emite uma onda eletromagnética nesta freqüência, de forma a transferir energia para os átomos de hidrogênio, colocando-os em um estado de excitação. Este fenômeno de transferência de energia através de freqüência eletromagnética específica é denominado ressonância;

iii) Detecção de radiofreqüência: quando os átomos excitados voltam ao estado normal, os mesmos emitem ondas eletromagnéticas na mesma freqüência que receberam $(63,8 \mathrm{MHz})$. O aparelho detecta a energia liberada, e através da utilização de gradientes magnéticos e da transformada bidimensional de Fourier determina a intensidade e a posição espacial de seus emissores.

Um equipamento para obtenção de imagens através da ressonância magnética é mostrada na Figura 1.1. O paciente permanece deitado dentro de um tubo (magneto), enquanto as fatias (slices) são adquiridas em cada aquisição (scan). Um slice é definido como a imagem bidimensional que representa uma fatia do cérebro (ou outra estrutura). O equipamento de ressonância magnética realiza uma série de aquisições a "cobrir" o volume de tecido, no caso o cérebro.

Para fins deste texto, as imagens de ressonância magnética podem ser de dois tipos. 


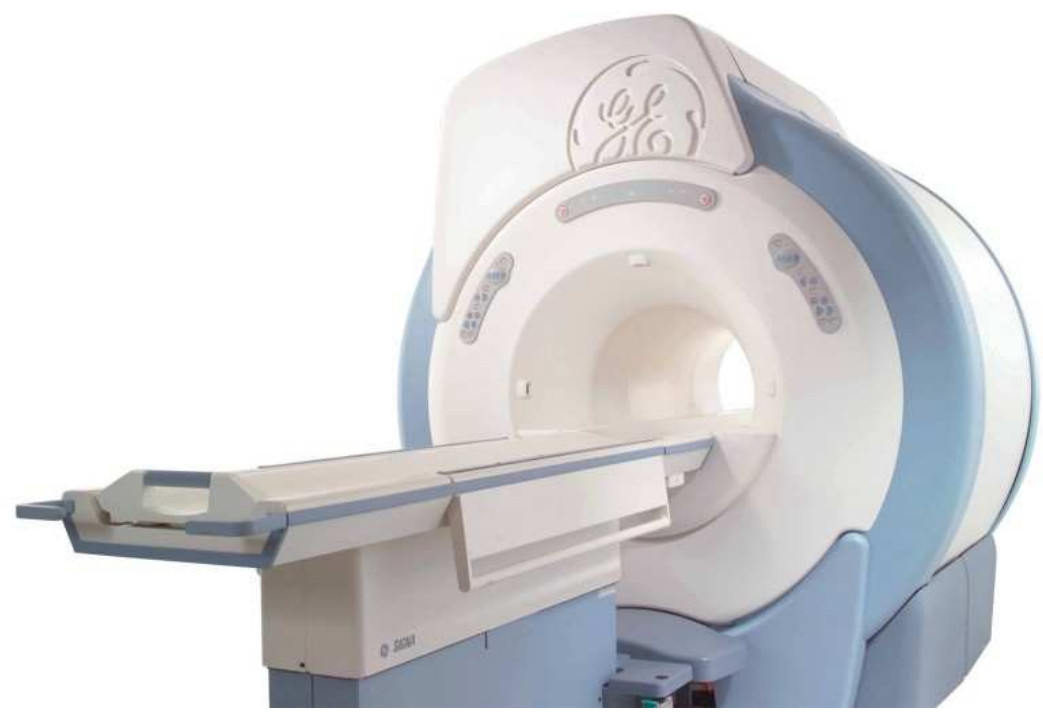

Figura 1.1: Equipamento de ressonância magnética.

Imagens funcionais e estruturais. As imagens estruturais são obtidas para a avaliação anatômica dos órgãos internos dos pacientes, como para a identificação de tumores ou aneurismas. Em geral, as imagens estruturais são de alta resolução espacial, porém o tempo de aquisição é de cerca de 5 minutos. As imagens de ressonância magnética funcional (RMf) são utilizadas para a identificação de alterações no fluxo sangüineo e oxigenação de uma determinada região, e veremos mais tarde que estão intimamente ligadas com a atividade neuronal. Como se deseja avaliar alterações temporais no fluxo sangüíneo, os volumes de RMf precisam ser obtidos em um curto tempo de aquisição, implicando uma menor resolução espacial deste tipo de imagem. As Figuras 1.2 e 1.3 ilustram uma imagem cerebral estrutural com reconstrução tridimensional e os slices que a compõem.

Sabemos que o transporte de oxigênio pela corrente sangüínea é realizado por um tipo especial de células denominadas hemácias. No interior das hemácias existe uma molécula chamada hemoglobina responsável pelo transporte de oxigênio, que de acordo com seu estado de oxigenação (oxihemoglobina e desoxihemoglobina) exibe propriedades magnéticas distintas. A desoxihemoglobina possui apenas cinco elétrons não pareados, apresentando a propriedade de paramagnetismo. As propriedades magnéticas no interior das hemácias torna-as diferentes em relação ao sangue na qual estão imersas (Thulborn et al. (1982), Amaro Junior (2000)). Dessa forma, a diferença no campo magnético será proporcional à concentração de deoxi-hemoglobina, possibilitando a geração do sinal BOLD (blood oxi- 


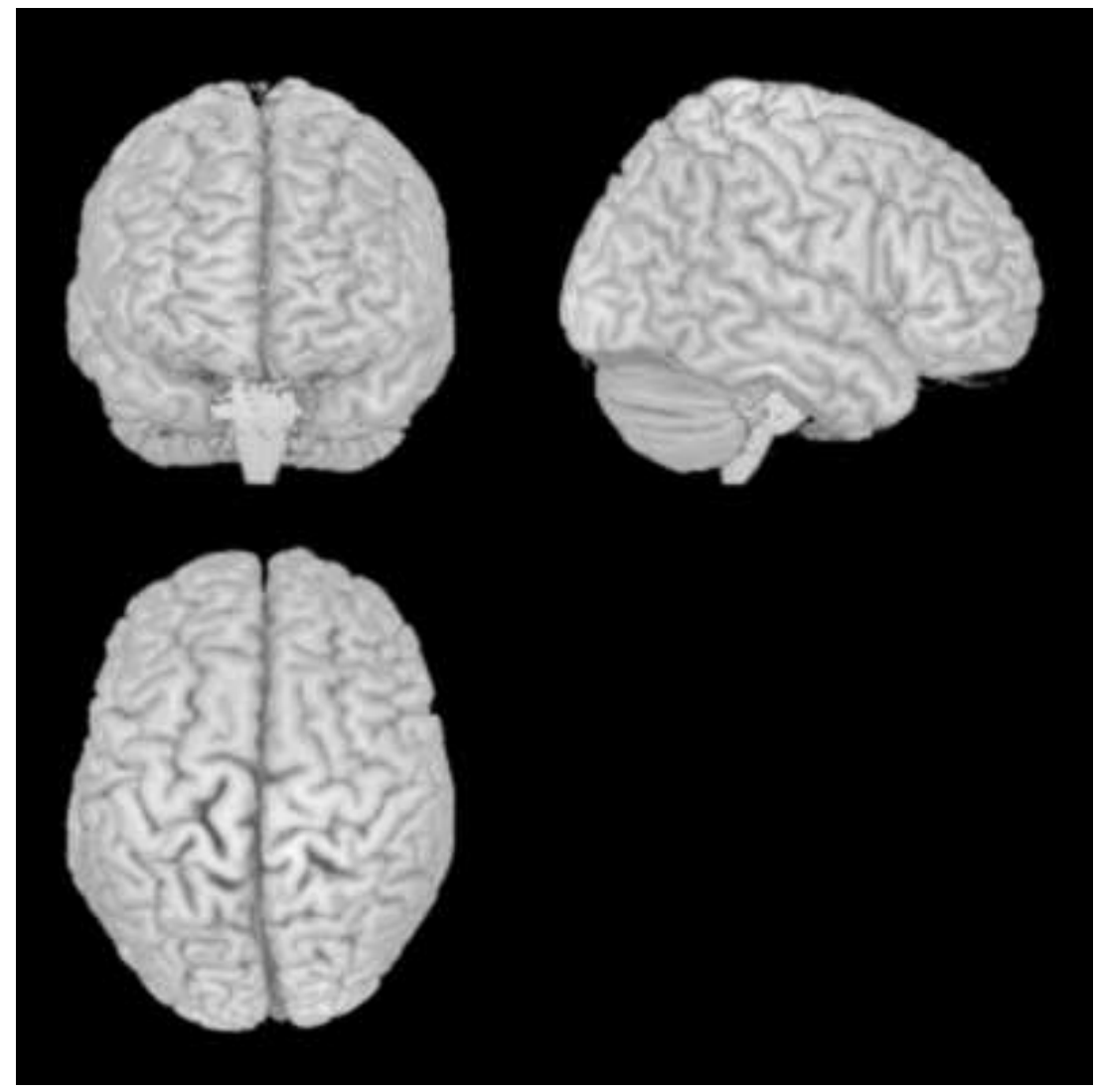

Figura 1.2: Imagem de RM estrutural tridimensional.

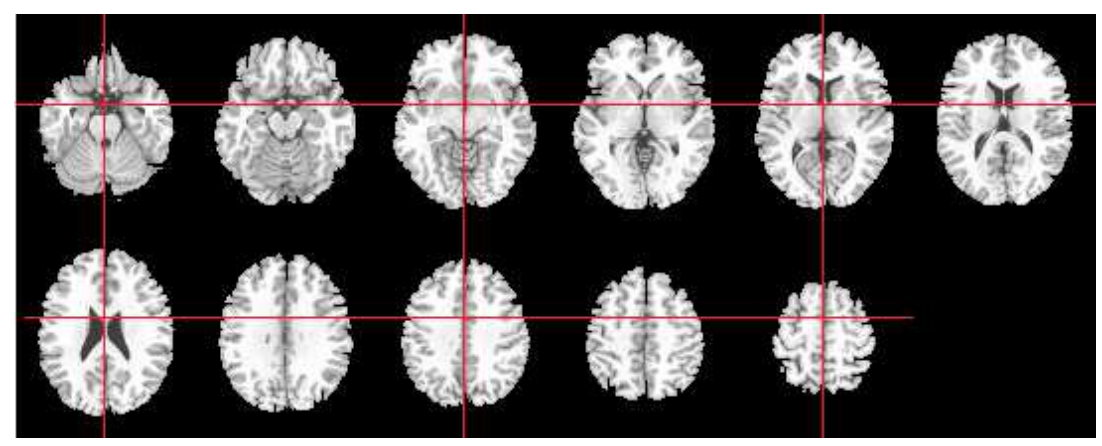

Figura 1.3: Imagem de RM estrutural por slices. 
genation level dependent, Ogawa et al. (1990)).

Quando determinada área cerebral é ativada, ou seja, existe um aumento na atividade neuronal (maior número de disparos elétricos ou atividade sináptica num determinado intervalo de tempo), verifica-se que há um aumento do fluxo sangüineo desta região. No entanto, o aumento do fluxo é desproporcionalmente maior em relação ao pouco aumento no consumo de oxigênio, de forma que a concentração de deoxi-hemoglobina cai drasticamente nas áreas ativadas. Um esquema de ativação pode ser descrito como a seguir

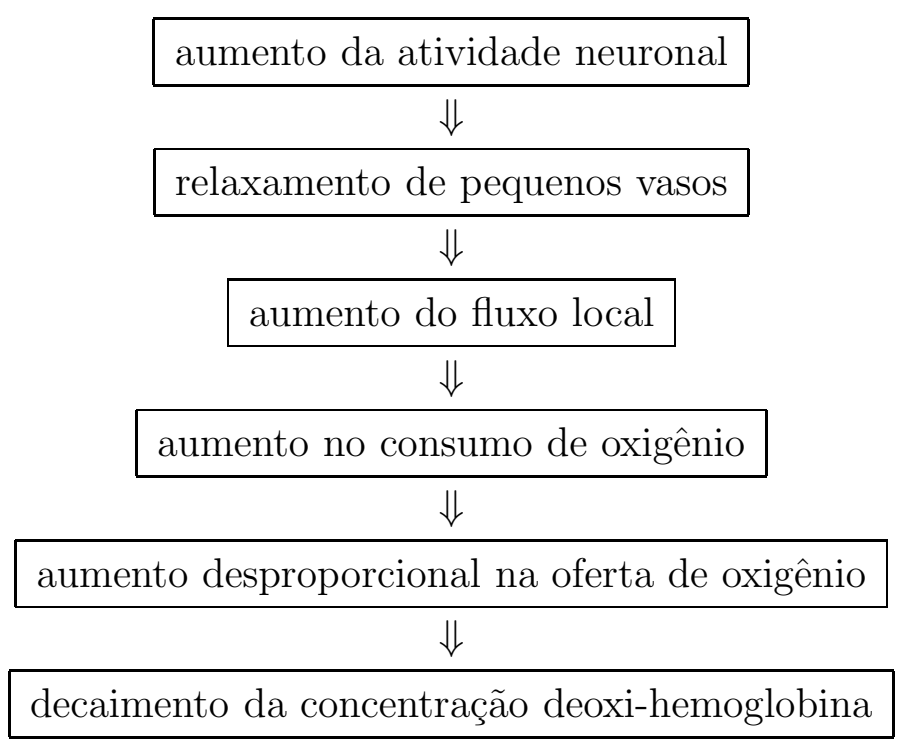

É importante ressaltar que mudanças na ativação cerebral não são diretamente observadas, mas apenas os efeitos de variações no fluxo sangüíneo e oxigenação microvascular. Dessa forma, no contexto de ressonâcia magnética, a atividade cerebral não é medida diretamente, mas através da análise da resposta hemodinâmica descrita pelo sinal BOLD. Segundo Logothetis (2003), o mecanismo de contraste BOLD foi implementado com sucesso em humanos, pequenos animais e recentemente até mesmo em primatas não humanos. 


\subsection{Processamento de Imagens de RMf}

Em termos de estrutura de dados, uma imagem de ressonância magnética (volume) é, basicamente, uma matriz tridimensional (linha $\times$ coluna $\times$ altura). Cada posição em um slice é descrito por coordenadas $x \times y$, ou seja, cada linha e coluna da matriz, e o valor de intensidade do sinal no voxel (pixel) é descrito pelo valor armazenado nesta posição. O termo pixel se refere à "célula" da imagem bidimensional. Voxel é o que o pixel representa no mundo real. A cada aquisição, adquiri-se um slice (matrix $x \times y$ ), cuja altura é descrita pela coordenada $z$. As dimensões (e.g., voxel de $3.75 \mathrm{~mm} \times 3.75 \mathrm{~mm} \times 5.5 \mathrm{~mm}$ ) as quais os valores de $x, y$ e $z$ correspondem são determinados na aquisição dos dados. Uma representação de uma matriz tridimensional está representada na figura 1.4.

Assim, sessão de RMf é uma série temporal de imagens, i.e., é uma seqüência de

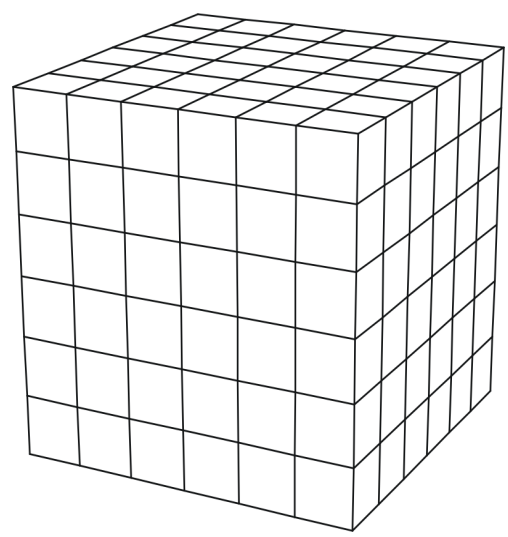

Figura 1.4: Matriz tridimensional de voxels

matrizes tridimensionais, e pode ser representada por uma estrutura tetradimensional da forma (linha $\times$ coluna $\times$ altura $\times$ tempo). O conjunto de sessões compõe o experimento. Assim, dadas as coordenadas $(\mathrm{x}, \mathrm{y}, \mathrm{z})$ de um voxel, podemos obter uma série temporal do sinal BOLD deste voxel, como mostra a figura 1.5

Toda análise de imagens é iniciada com uma série de pré-processamentos dos dados, de forma a remover ruídos e componentes inerentes ao processo de aquisição. A primeira transformação aplicada aos dados é efetuada já durante o processo de aquisição. É aplicada uma transformada bidimensional de Fourier, de forma que as imagens coletadas (espaço $K$ ) tomem efetivamente a forma de cérebros. A figura 1.6 ilustra este procedimento. 


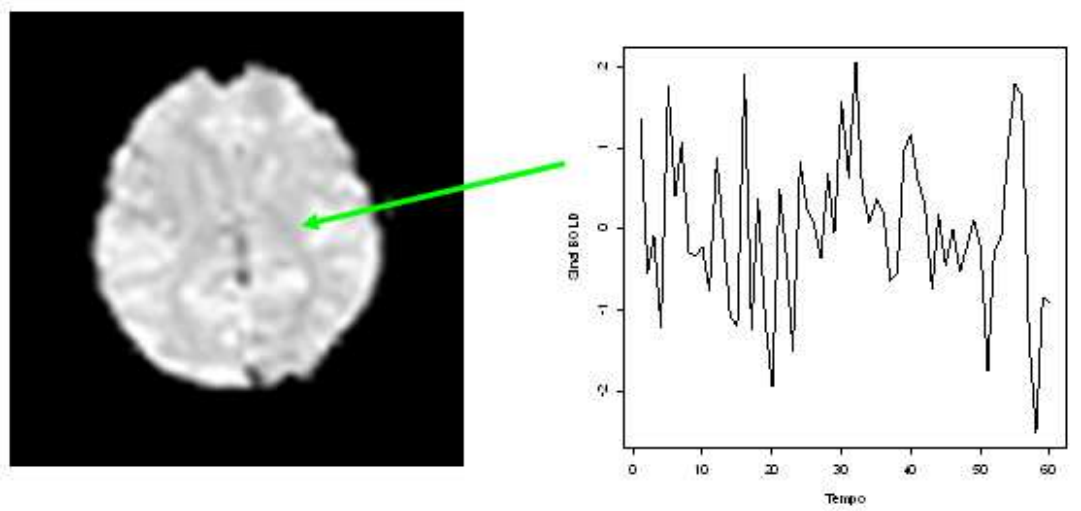

Figura 1.5: Sinal BOLD

Em seguida, são aplicadas transformações de realinhamento espacial, temporal e nor-
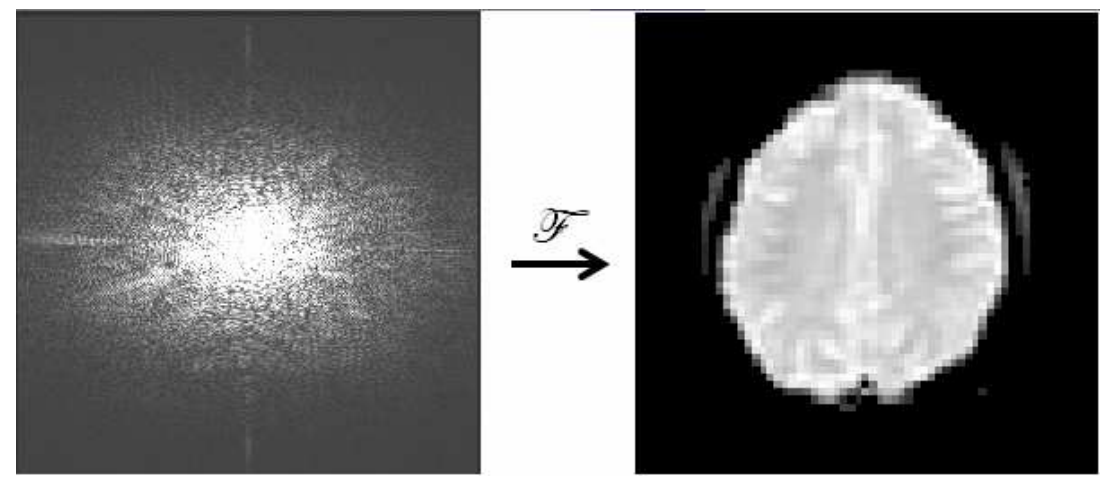

Figura 1.6: Aplicação da Transformada de Fourier

malizações espaciais, que buscam corrigir componentes artefatuais de movimento, e tornar comparáveis dados de mais de um indivíduo.

\subsubsection{Realinhamento}

O primeiro passo no processamento pós-aquisição dos dados é o realinhamento espacial, de forma a reduzir o efeito de componentes da série temporal devidos ao movimento da cabeça do paciente durante a sessão (run) de coleta das imagens. Por mais que a cabeça do indivíduo esteja fixada, os movimento respiratórios e batimentos cardícacos também podem resultar em deslocamentos milimétricos, que podem prejudicar as análises sub- 
seqüentes. Dessa forma, é uma prática comum realinhar todos os volumes adquiridos em relação ao primeiro (ou média).

O processo de realinhamento envolve a estimação de parâmetros, de forma a minimizar a soma de quadrados da diferença entre o volume de referência e os subseqüentes. Dessa forma, aplicam-se transformações de rotação e translação (corpo rígido) nas imagens utilizando os parâmetros estimados. Um exemplo de de realinhamento é mostrado na Figura 1.7 .

Um outro fator importante que também deve ser considerado, é o tempo e esquema

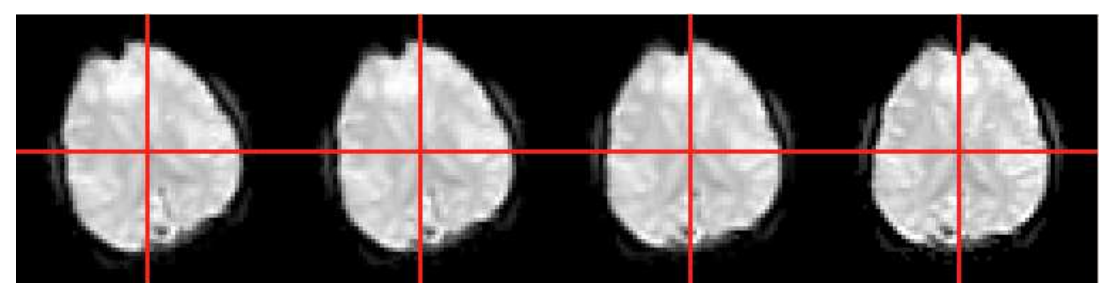

(a)

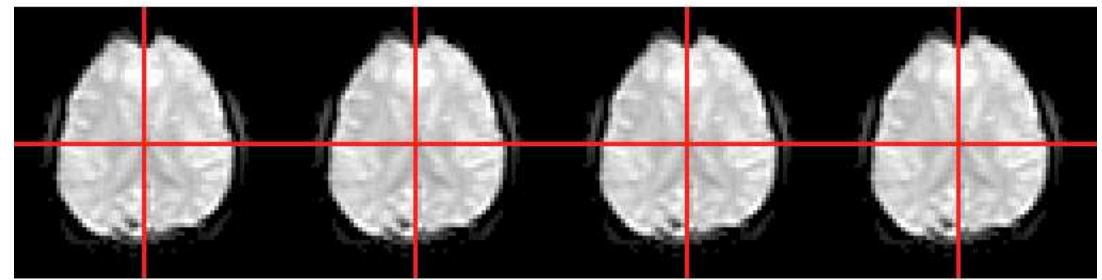

(b)

Figura 1.7: Realinhamento: (a) Série temporal de slices antes do alinhamento. (b) Série temporal de slices após o alinhamento.

de aquisição de slices. Exemplificando, se o tempo de aquisição de um volume com 20 slices é de 2 segundos, a defasagem temporal do primeiro slice adquirido e o último, será de quase 2 segundos. Assim, é necessária fazer uma reamostragem dos dados, visando realinhar temporalmente a aquisição dos slices. Na maior parte dos estudos, são utilizadas interpolações temporais através de funções sinc's ou splines cúbicas.

\subsubsection{Normalização Espacial}

Caso haja a necessidade de comparação de imagens coletadas em indivíduos diferentes (e.g., análise de um grupo como um todo), ou com um espaço anatômico de um atlas (um 
modelo de cérebro médio de uma população), as imagens são deformadas para um modelo espacial ("template"). Emboram as imagens já estejam alinhadas, ainda existem componentes de variações espaciais, pois em estudos com mais de um indivíduo, provavelmente os cerébros de indivíduos distintos não terão as mesmas dimensões nem as mesmas formas. Além disso, até mesmo volumes adquiridos em um mesmo indivíduo podem possuir variações caso os volumes sejam tomados em equipamentos ou sessões diferentes, ou caso as configurações do processo de aquisição sejam distintas.

Considerando-se os aspectos de variações mencionados, as imagens são transformadas para um modelo (template), em acordo com algum espaço anatômico pré-estabelecido. Em geral, utiliza-se o espaço anatômico de Talairach e Tournoux (1988). Além disso, esta padronização é importante para descrever as áreas em termos de coordenadas tridimensionais, possibilitando uma unificação dos resultados de diversos experimentos. Na maior parte dos casos, utilizam-se deformações espaciais obtidas através de procedimentos de mínimos quadrados ou interativos (maximizando-se a correlação ou informação mútua da imagem distorcida e o template). A Figura 1.8 mostra um modelo (padrão do software XBAM, www.brainmap.co.uk) utilizado para normalização para o espaço de Talairach e Tournoux (1988).

Além das transformações espaciais, muitas vezes também são utilizados procedimen-

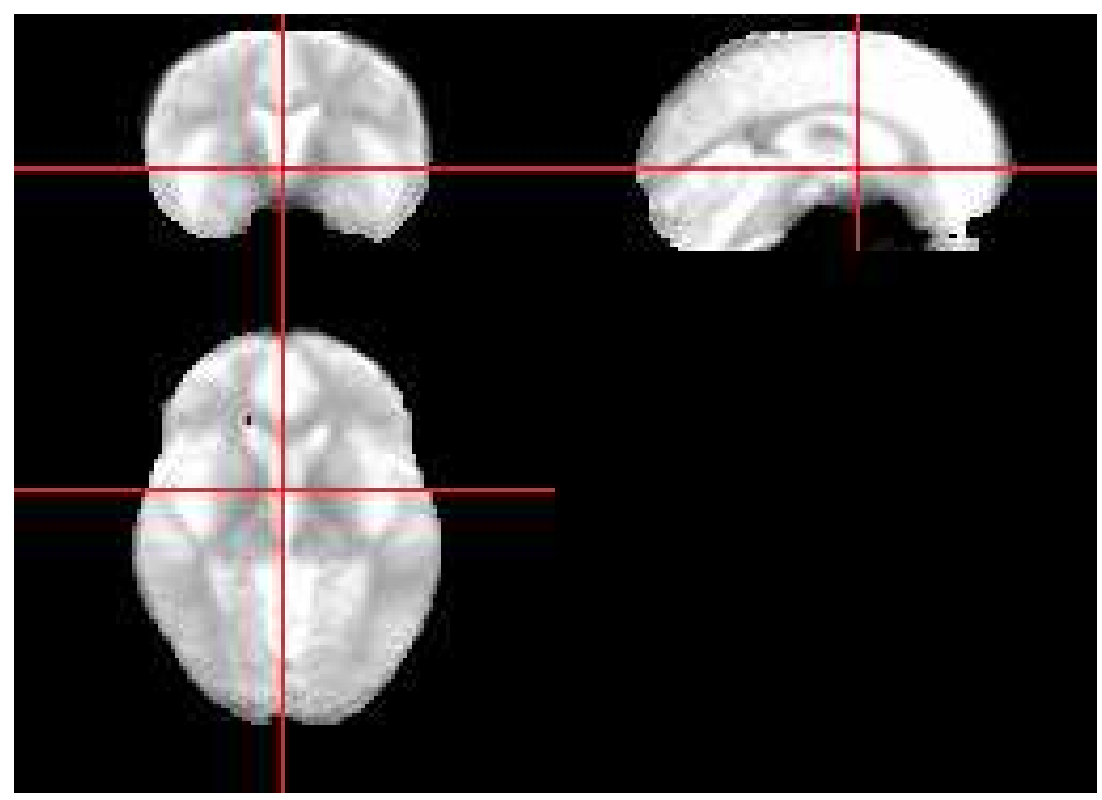

Figura 1.8: Modelo para normalização espacial. 
tos de suavização espacial dos slices, através da convolução das imagens por um kernel bidimensional gaussiano. Alguns motivos que justificam a suavização são: eliminar componentes (ruídos) que não são plausíveis segundo o contexto neurofisiológico, a suavização induz a normalidade dos resíduos de modelos estatísticos, e uma melhor homogeneização espacial para experimentos com vários indivíduos.

\subsection{Mapeamento Cerebral}

O mapeamento de funções cerebrais consiste na identificação de áreas relacionadas à funções específicas. Diversos estudos mostram que o cérebro não possui uma dinâmica caótica ou ilógica de funcionamento, mas é composto por módulos funcionais (agrupamento de neurônios especializados). Determinadas regiões cerebrais, isto é, determinados conjuntos de neurônios adjacentes, são responsáveis pela realização de funções específicas, tais como a visão, audição, movimento, associação, etc. A Figura 1.9 mostra algumas áreas cerebrais, as quais sabemos que estão relacionadas à algumas funções básicas.

O mapeamento cerebral estatístico refere-se ao teste de hipóteses sobre a atividade

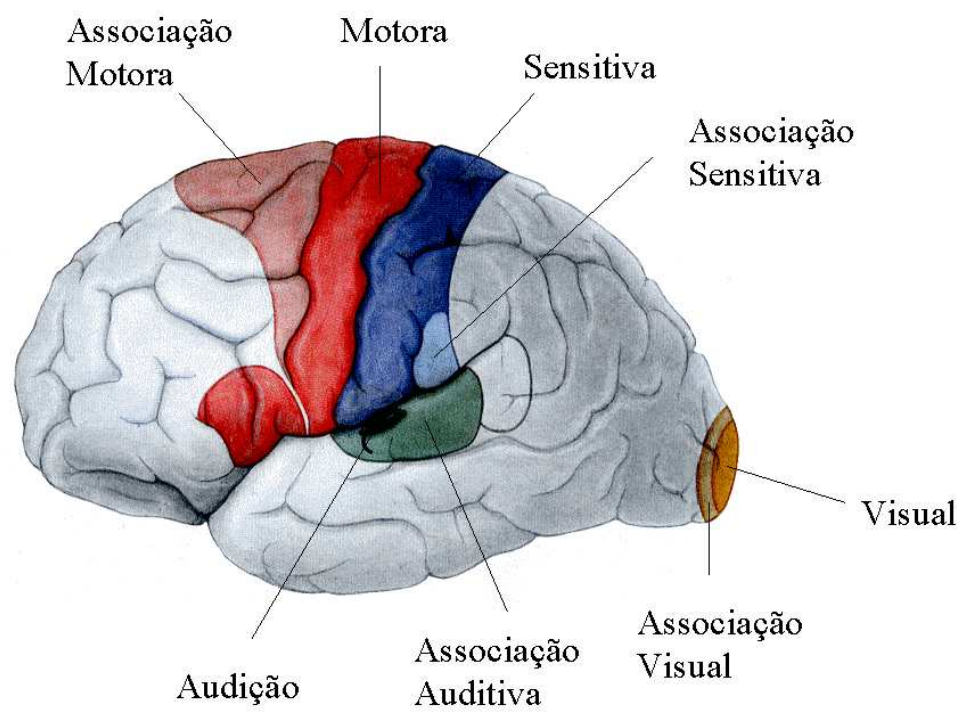

Figura 1.9: Funções cerebrais básicas.(Adaptado de Sobota, 1993)

neuronal de uma determinada região, relacionada a uma função cerebral específica. As- 
sim, através de testes univariados de média, é possível verificar quais são as áreas cerebrais (descritas em termos de voxels) estatisticamente ativadas durante a realização de uma determinada tarefa, ou ao receber determinados estímulos. Em outra palavras, podemos verificar a ativação cerebral testanto as diferenças de médias do sinal BOLD, quando o paciente é submetido a estímulos ou realiza tarefas específicas como mover os dados, ler, etc.

\subsubsection{Experimentos e Paradigmas}

Um dos principais elementos no estudo de mecanismos neurocognitivos é o protocolo de experimento utilizado. A forma como o experimento foi desenvolvido é fundamental na especificação de procedimentos de análises dos dados, e também para a interpretação dos resultados. O conjunto de atividades realizadas durante uma sessão experimental de estudo de neuroimagem é denominado paradigma. Nos estudos de neuroimagem funcional, dependo da forma como são apresentados os estímulos, os paradigmas mais utilizados podem ser classificados basicamente em três classes: nulo (resting state), blocos (block design) e relacionado a eventos (event related).

O paradigma nulo, usualmente denotado por resting state, é o tipo mais simples de um experimento neurocognitivo. Neste paradigma, o indivíduo permanece dentro do equipamento de ressonância magnética enquanto as imagens são adquiridas, mas não há nenhuma apresentação de estímulos. Assim sendo, o sinal BOLD deve representar o funcionamento cerebral quando não há tarefas específicas. No entanto, este tipo de estudo é difícil de ser avaliado devido a fatores como pensamentos, emoções, memória, etc... que podem até mesmo estar mais presentes em uma situação de descanso do que em atividade (foco de atenção).

O paradigma com desenho de blocos envolve a apresentação de estímulos repetidos e condições seqüenciais. O indivíduo é orientado a realizar uma atividade específica durante um determinado intervalo de tempo (epoch). No intervalo de tempo seguinte, o indivíduo realiza outra tarefa específica. Assim, é possível quantificar as diferenças na média do sinal BOLD para estas duas condições. Além disso, o paradigma com desenho de bloco pode envolver duas ou mais condições, e também alternância periódica de condições. Um exemplo deste paradigma é a realização periódica de duas atividades. Durante 20 segundos 
um indivíduo permanece em estado de descanso (atividade A). Em seguida, permanece 20 segundos recebendo um estímulo visual (atividade B). No intervalo seguinte, o indivíduo volta a permanecer em descanso, e repete-se este ciclo um certo número de vezes. É importante mencionar que os sinais obtidos em desenhos de blocos possuem uma maior razão sinal-ruído, de forma que o nível de ativação em cada condição é mais precisamente estimado.

Um experimento com paradigma relacionado a eventos também envolve a apresentação de estímulos. Uma série de estímulos (0, 1 ou 2 segundos de duração) de interesse ocorrem de forma espaçada no tempo. Assim, pode-se avaliar a evolução do sinal BOLD das regiões cerebrais durante a apresentação de estímulo curtos. Um exemplo de paradigma relacionado a eventos é um experimento onde um indivíduo permanece em descanso, e subitamente, recebe um rápido estímulo visual.

\subsubsection{Alguns Modelos}

O modelo mais simples para a ativação cerebral consiste em um modelo de regressão linear, considerando o sinal BOLD como variável resposta e o estado de estímulos como variável explicativa. Assim considere um experimento de RMf periódico, no qual é realizada uma tarefa A e outra B, repetindo-se o ciclo diversas vezes. Na maior parte dos casos, o objetivo deste tipo de estudo é a detecção da diferença no nível de ativação entre os dois estados. Considerando-se um modelo de regressão linear simples, temos

$$
X_{t}=\beta a_{t}+\epsilon_{t}
$$

onde $a_{t}=1$ se o paciente está realizando a tarefa $\mathrm{A}$ no instante $t$ e $a_{t}=-1$ se está realizando a tarefa B. É evidente que o sinal $X_{t}$ deve ser normalizado para média nula e ter tendência removida, se existir. O estimador de mínimos quadrados ordinários da diferença da média do nível de ativação entre os dois estados $\beta$ é dado por

$$
\hat{\beta}=\frac{1}{T}\left(\sum_{A} X_{t}-\sum_{B} X_{t}\right),
$$

e aplicando testes de hipóteses tradicionais para modelos de regressão linear simples, podemos testar se o efeito de ativação é estatisticamente significativo. 
No entanto, sabemos que existe um atraso entre a resposta hemodinâmica e a mudança de estado ou tarefa. Dessa forma, Bandetini et al. (1993) sugeriram a utilização de um atraso em $a_{t}$. Além disso, considerando que $a_{t}$ pode assumir outras formas além da função retângulo (boxcar), pode-se considerar um modelo mais geral dado por

$$
X_{t}=\beta f(t ; \theta)+\epsilon_{t}
$$

onde $f$ é uma função, com parâmetros de formas descritos no vetor $\theta$. Considerando o comportamento fisiológico do neurônio e o mecanismo de resposta hemodinâmica, Friston et al. (1994) sugerem a utilização de uma função Poisson $f$ dada por

$$
f(s ; \lambda)=\frac{\lambda^{s} e^{-\lambda}}{s !}
$$

onde o parâmetro $\lambda$ determina o atraso do pico entre a mudança de estado e resposta hemodinâmica. Mais tarde, Lange e Zeger (1997) propuseram uma função mais flexível, utilizando uma função gama $\Gamma(\lambda, u)$ dada por

$$
f(s, \lambda, u)=\frac{1}{\Gamma(u)} \lambda^{u} s^{u-1} e^{-\lambda s}
$$

com $u, \lambda \geq 0$, possibilitando um ajuste na forma e na escala da função. No entanto, a estimação dos parâmetros de forma e escala é computacionalmente intensa, devido a nãolinearidade. Assim, baseando-se nas séries de Fourier, Bullmore et al. (1996) sugeriram a utilização de uma expansão truncada em funções senos e cossenos dada por

$$
X_{t}=\gamma_{1} \sin (\lambda t)+\delta_{1} \cos (\lambda t)+\gamma_{2} \sin (2 \lambda t)+\delta_{2} \cos (2 \lambda t)+\gamma_{3} \sin (3 \lambda t)+\delta_{3} \cos (3 \lambda t)+\epsilon_{\mathrm{t}}(1.6)
$$

onde $\lambda$ é a freqüência angular. Note que para este modelo os coeficientes podem ser obtidos através de uma regressão linear múltipla. Na prática, utiliza-se funções hemodinâmicas pré-estabelecidas, incluindo expansões de Volterra ou derivadas.

Além disso, em grande parte dos casos, verifica-se que os resíduos das regressões são autocorrelacionados, de forma que o estimador por mínimos quadrados ordinários não é mais ótimo (Best Linear Unbiased Estimator). Nestes casos, utiliza-se o já conhecido método de Cochrane-Orcut para erros correlacionados. No entanto, um dos métodos mais populares para detecção de ativação cerebral é o wavestrap. O wavestrap é um método não paramétrico para a avaliação de quantis, e foi introduzido em RMf por Bullmore et al.(2001). Os métodos não paramétricos são freqüentemente utilizados nos casos em que 
a distribuição dos erros aleatórios do modelo não é conhecida e o número de amostras é grande. Como a suposição de normalidade pode não ser válida em alguns experimentos de RMf, a utilização de uma metodologia mais flexível torna-se extremamente atrativa. Dessa forma, com o apoio computacional, algoritmos do tipo bootstrap têm sido constantemente aplicados para uma derivação aproximada de distribuições. No entanto, um obstáculo para a utilização de métodos de reamostragem em RMf é a existência de autocorrelação nos resíduos. Em outras palavras, os erros aleatórios não são independentes entre si, de forma que a utilização de métodos de permutação eliminaria a autocorrelação dos erros. Assim, as amostras bootstrap já não teriam as mesmas propriedades do sinal BOLD, invalidando os resultados da análise.

Embora seja possível aplicar métodos de reamostragem se considerarmos o procedimento de Cochrane and Orcut, Bullmore et al.(2001) verificaram que especificações incorretas da estrutura de autocorrelação pode produzir resultados viesados. Assim, Bullmore et al.(2001) sugeriram a utilização do wavestrap, que não necessita de pré-especificações da estrutura de autocovariância. Basicamente, o wavestrap é um algoritmo de reamostragem realizado no domínio de ondaletas (wavelets), de forma que a autocorrelação das séries reamostradas é a mesma da série original. O procedimento wavestrap pode ser sumarizado por

i) Decomposição da série original em coeficientes de ondaletas;

ii) Reamostragem dos coeficientes dentro de cada escala;

iii) Transformada inversa de ondaletas.

É interessante verificar que a estrutura de covariância é invariante a permutações dos coeficientes de ondaletas em cada escala. Além disso, no domínio de ondaletas, os coeficientes são não-correlacionados, permitindo a utilização do bootstrap. Um exemplo considerando uma série simulada está descrito na Figura 1.10.

Assim, utilizando o wavestrap ou outros modelos descritos anteriormente é possível estimar e realizar testes estatísticos a respeito do nível de ativação de um determinado voxel. No entanto, sabemos que o erro do tipo I é inerente aos testes de hipóteses. Assim, como são realizados testes de hipóteses para cada voxel, esperamos um número de ativações espúrias. Devido às características de amostragem do sinal e pré-processamento das imagens a ativação de um único voxel isolado pode ser um caso de ativação espúria. Assim, após os teste de ativação, é necessário verificar quais ativações formam grupos (clusters) consistentemente. 

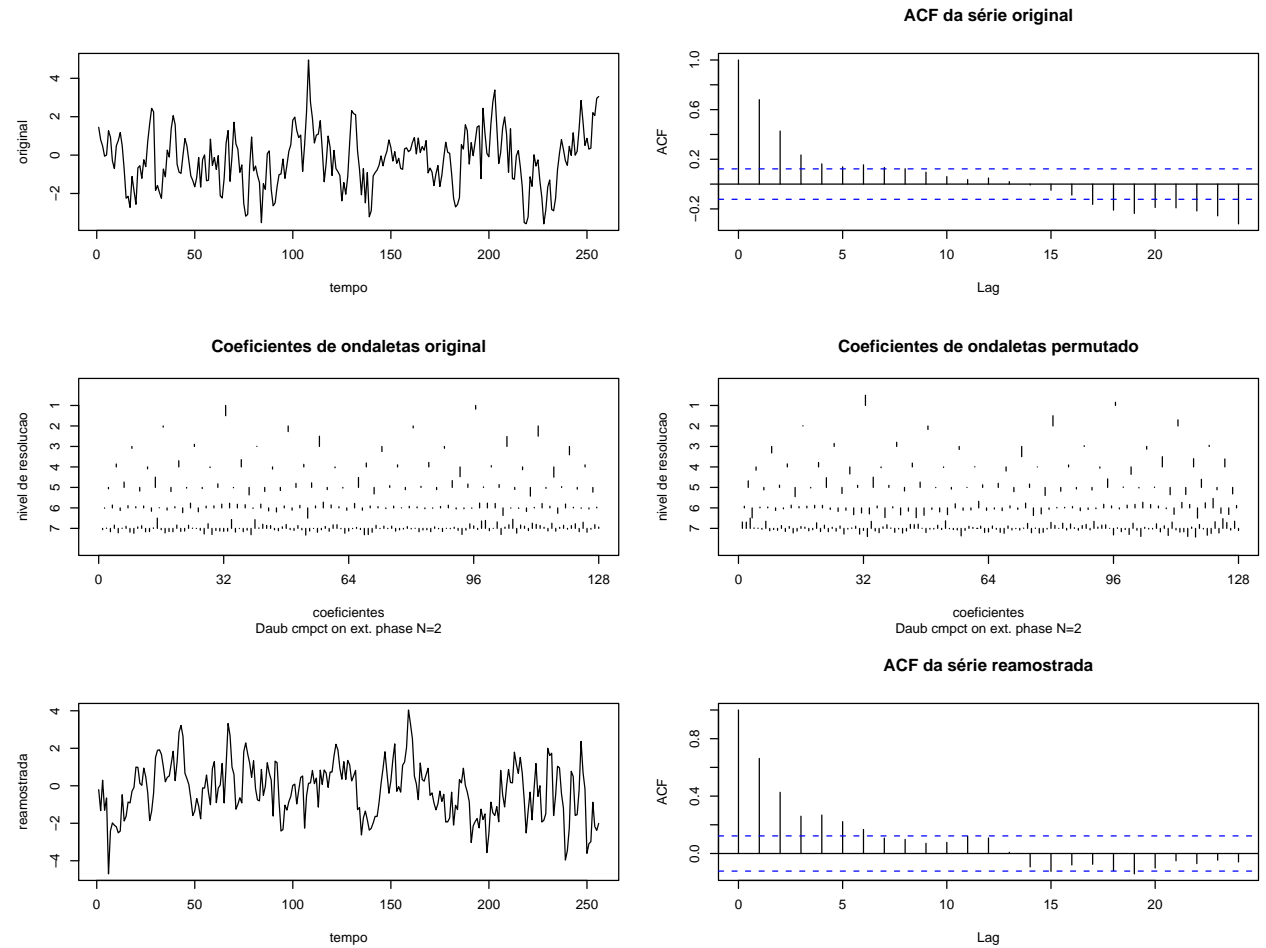

Figura 1.10: Exemplo de aplicação do algoritmo wavestrap.

A maioria dos métodos de detecção de ativações espúrias utilizam a informação dos voxels próximos ao voxel de interesse, buscando agrupamentos (clusters) de voxels ativados. Worsley e Friston (1995) sugeriram a utilização da teoria dos campos gaussianos, considerando que a imagem das estatísticas é uma coleção de variáveis com distribuição conjunta gaussiana, e derivaram uma expressão aproximada de um único voxel ser maior que um limiar, sobre a hipótese de que não há ativação. Um outro método freqüentemente utilizado é simplesmente a convolução das imagens de estatísticas por um kernel bidimensional, com tamanho de janela aproximadamente de 2 ou 3 voxels.

Aplicação sobre dados reais de algumas das metodologias descritas neste capítulo são apresentadas no capítulo 6 . 


\section{Ondaletas}

Neste capítulo serão apresentados alguns conceitos básicos sobre a teoria de ondaletas (wavelets), uma ferramenta cujas aplicações em diversas áreas do conhecimento vêm crescendo rapidamente. Se por um lado a teoria de Fourier é útil e eficiente no contexto de séries estacionárias, por outro o caráter de não localização temporal de sua transformada dificulta a análise de séries temporais não-estacionárias. No entanto, veremos que a transformada de ondaletas é localizada no tempo e na escala, permitindo uma forma elegante de análise de séries não-estacionárias.

\subsection{Funções de Ondaletas}

\subsubsection{Introdução}

Assim como as bases de Fourier, splines, Walsh, etc, as bases de ondaletas são utilizadas para gerar espaços de funções. As bases de Fourier são ótimas para a análise de processos estacionários, mas as funções trigonométricas não são localizadas temporalmente, de forma que a alteração de apenas uma única observação da série, alterará todos os coeficientes da transfomada de Fourier. As bases de funções splines são localizadas no tempo, porém são eficientes apenas em espaços homogêneos, onde não existe a necessidade de uma janela adaptativa. Uma característica interessante das ondaletas é a localização em tempoescala, permitindo a análises de processos não-estacionários e adaptatividade em espaços não-homogêneos como os de Besov. 
A idéia básica da teoria das ondaletas é gerar espaços a partir de dilatações (escala) e translações (tempo) de uma única função $\psi$, denominada geratriz ou ondaleta mãe. Assim, desejamos aproximar funções do $L^{2}(\mathbb{R})$ utilizando combinações lineares de funções pertencentes a este espaço. A base de funções é geradas pelas funções pertencentes a classe

$$
\Psi=\left\{\psi_{a, b}(x)=|a|^{-1 / 2} \psi\left(\frac{x-b}{a}\right), a, b \in \mathbb{R}, a \neq 0\right\}
$$

onde a ondaleta mãe $\psi$ deve satisfazer as seguintes propriedades:

i) $\int_{-\infty}^{\infty} \psi(t) d t=0$ (condição de admissibilidade);

ii) $\int_{-\infty}^{\infty}|\psi(t)| d t<\infty$;

iii) $\int_{-\infty}^{\infty} \frac{|\hat{\psi}(\lambda)|}{|\lambda|} d \lambda<\infty$, onde $\hat{\psi}(\lambda)$ é a transformada de Fourier de $\psi(t)$;

iv) $\int_{-\infty}^{\infty} t^{j} \psi(t) d t=0, j=0,1,2, \ldots, r-1$, para algum $r \geq 1$ e $\int_{-\infty}^{\infty}\left|t^{r} \psi(t)\right| d t<\infty$.

Considere uma base gerada por $\psi$, através de dilatações binárias e translações diádicas, da forma

$$
\psi_{j, k}(t)=2^{j / 2} \psi\left(2^{j} t-k\right)
$$

com $j, k \in \mathbb{Z}$. Um resultado de grande importância é que qualquer função $g(t)$ com $\int_{-\infty}^{\infty} g(t)^{2} d t<\infty$, pode ser representada através da expansão em ondaletas dada por

$$
g(t)=\sum_{j=-\infty}^{\infty} \sum_{k=-\infty}^{\infty} c_{j, k} \psi_{j, k}(t)
$$

onde a convergência é dada em termos de média quadrática, e os coeficientes de ondaletas $c_{j, k}$ são obtidos através do seguinte produto escalar

$$
c_{j, k}=<g, \psi_{j, k}>=\int_{-\infty}^{\infty} g(t) \psi_{j, k}(t) d t
$$

Como sabemos que o produto escalar não depende do domínio na qual é aplicado, analogamente à análise de Fourier, a relação de Parseval da energia é válida, ou seja,

$$
\int_{-\infty}^{\infty} g(t)^{2} d t=\sum_{j} \sum_{k} c_{j, k}^{2} .
$$

Como cada coeficiente $c_{j, k}$ está associado a uma função $\psi_{j, k}$ localizada no tempo-escala (índices $j$ e $k$ ), é possível obter uma mapeamento da energia do sinal tanto temporalmente quanto na escala, permitindo até mesmo a análise de séries não-estacionárias. 
Alguns exemplos de ondaletas (ver Figura 2.1):

i) Ondaleta de Haar:

$$
\psi(t)=\left\{\begin{array}{cl}
1 & , 0 \leq t<1 / 2 \\
-1 & 1 / 2 \leq t<1 \\
0 & , \text { c.c. }
\end{array}\right.
$$

ii) Chapéu Mexicano:

$$
\psi(t)=\left(1-t^{2}\right) \exp \left(\frac{-t^{2}}{2}\right)
$$

iii) Ondaleta de Shannon:

$$
\psi(t)=\frac{\operatorname{sen}\left(\frac{\pi t}{2}\right)}{\frac{\pi t}{2}} \cos \left(\frac{3 \pi t}{2}\right) .
$$

iv) Ondaleta de Morlet:

$$
\psi(t)=\exp (i w t) \exp \left(-t^{2} / 2\right)
$$

Uma outra forma de gerar ondaletas, é considerar funções estritamente ortogonais $\phi$ solução da equação

$$
\phi(t)=\sqrt{2} \sum_{k} l_{k} \phi(2 t-k)
$$

onde

$$
l_{k}=\sqrt{2} \int_{-\infty}^{\infty} \phi(t) \phi(2 t-k) d t
$$

Assim como no caso das funções $\psi$, através de dilatações e translações de $\phi$ (ondaletapai ou função escala), podemos gerar uma família ortonormal da forma

$$
\phi_{j, k}(t)=2^{j / 2} \phi\left(2^{j} t-k\right)
$$

com $j, k \in \mathbb{Z}$. Assim, é possível obter funções $\psi$ que satisfazem todas propriedades descritas anteriormente, através de um filtro em $\phi$, isto é,

$$
\psi(t)=\sqrt{2} \sum_{k} h_{k} \phi(2 t-k)
$$



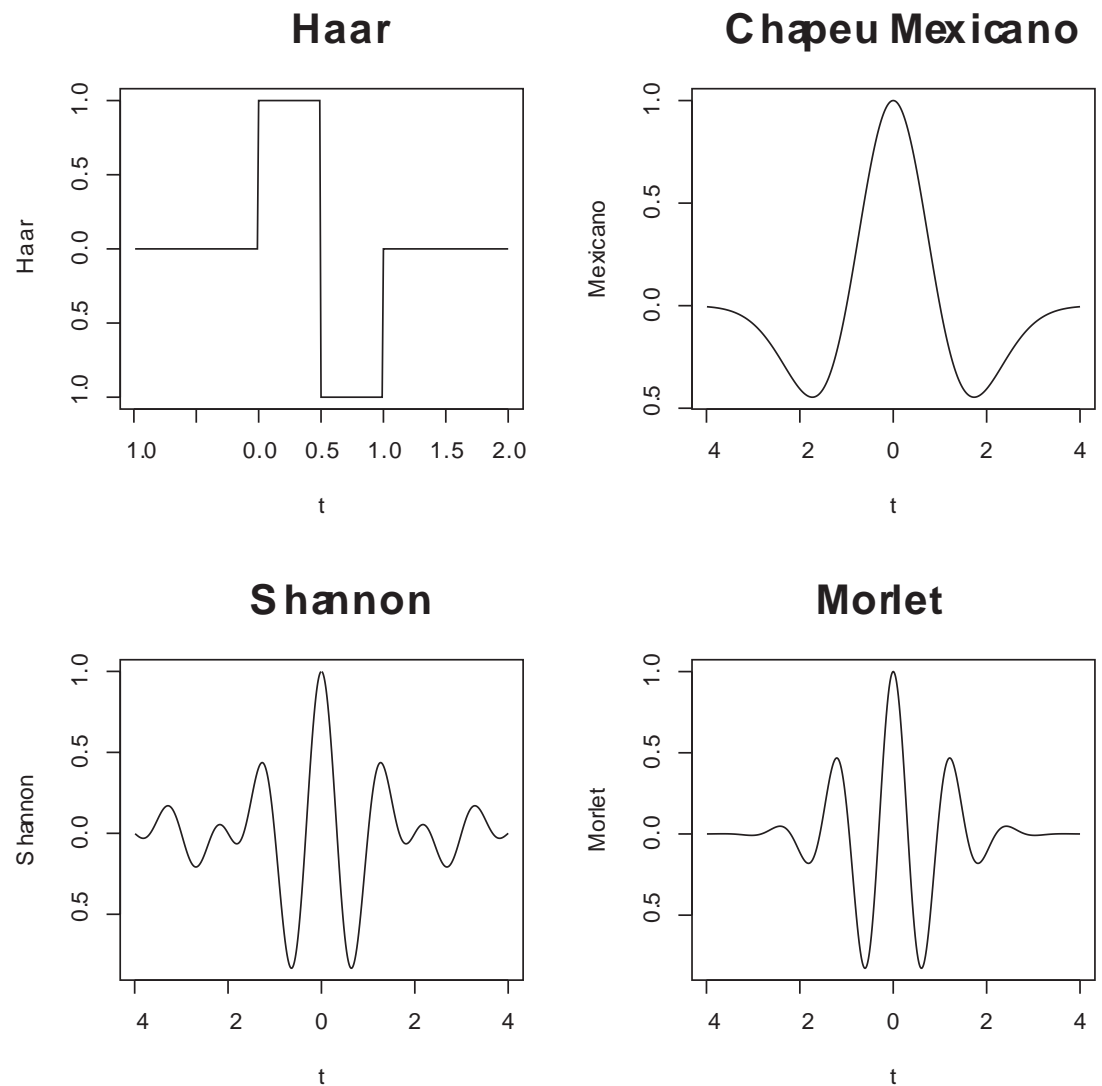

Figura 2.1: Ondaletas de Haar, chapéu mexicano, Shannon e Morlet. 
com

$$
h_{k}=\sqrt{2} \int_{-\infty}^{\infty} \psi(t) \phi(2 t-k) d t
$$

onde temos a quadrature mirror filter relation dada por $h_{k}=(-1)^{k} l_{1-k}$. Se observarmos os filtros $l_{k}$ e $h_{k}$ no enfoque da análise de Fourier, verifica-se que $l_{k}$ é um filtro passa-baixa, e $h_{k}$ e um filtro passa-alta. Além disso, podemos definir um sistema ortonormal da forma

$$
\left\{\psi_{j, k}, \phi_{j, k}, j, k \in \mathbb{Z}\right\}
$$

Logo, dados os coeficientes dos filtros, qualquer função $g(t) \operatorname{com} \int_{-\infty}^{\infty} g(t)^{2} d t<\infty$, pode ser representada através da expansão em ondaletas dada por

$$
g(t)=\sum_{k} c_{j_{0}, k} \phi_{j_{0}, k}(t)+\sum_{j \geq j_{0}} \sum_{k} d_{j, k} \psi_{j, k}(t)
$$

onde os coeficientes da expansão são dados por

$$
\begin{aligned}
c_{j 0, k} & =\int_{-\infty}^{\infty} g(t) \phi_{j_{0}, k}(t) d t \\
d_{j, k} & =\int_{-\infty}^{\infty} g(t) \psi_{j, k}(t) d t
\end{aligned}
$$

e $j_{0}$ é muitas vezes assumido como zero.

Algumas das ondaletas mais utilizadas são as ondaletas com suporte compacto de Daubechies (1992), construídas através dos filtros passa-alta e passa-baixa. A Figura 2.2 e 2.3 mostra as ondaletas Daublets de dupla fase extrema (Double Extreme Phase) e as menos assimétricas (Least Asymetric), respectivamente.

\subsubsection{Análise Multirresolução}

Como foi visto, as ondaletas são bem localizadas na escala, sendo portanto, uma possível ferramenta para a análise multirresolução (AMR). Em outras palavras, é como se pudéssemos observar os dados em diferentes resoluções ou magnificações, permitindo desprezar ou incluir detalhes. Em termos formais, a AMR é uma seqüência crescente de espaços fechados $\left(V_{i}\right)$, com as seguintes propriedades:

i) $\ldots \subset V_{-1} \subset V_{0} \subset V_{1} \subset \ldots$

ii) $L^{2}(\mathbb{R})=\overline{\cup_{j} V_{j}}$. 

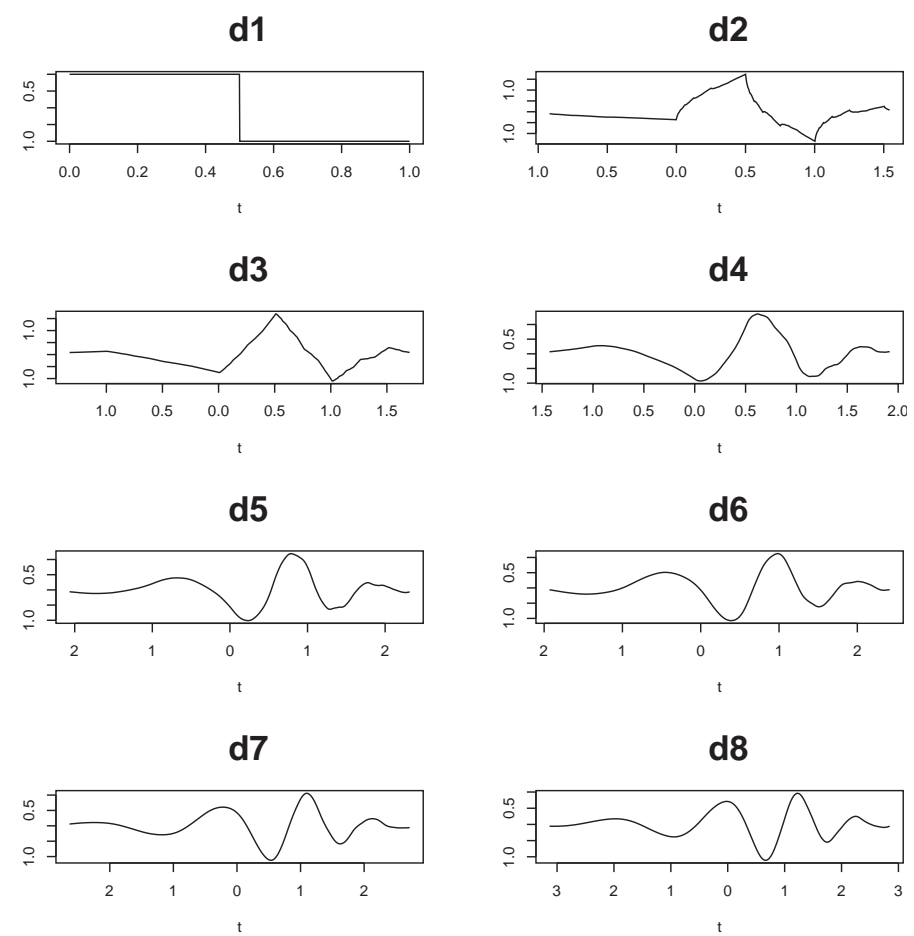

Figura 2.2: Ondaletas Daublets de Dupla Fase Extrema.
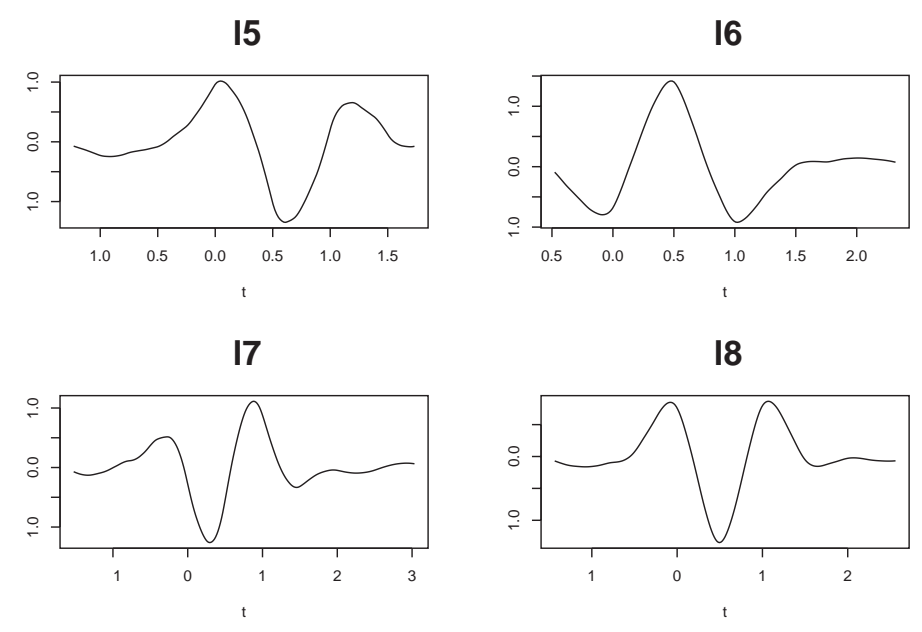

19
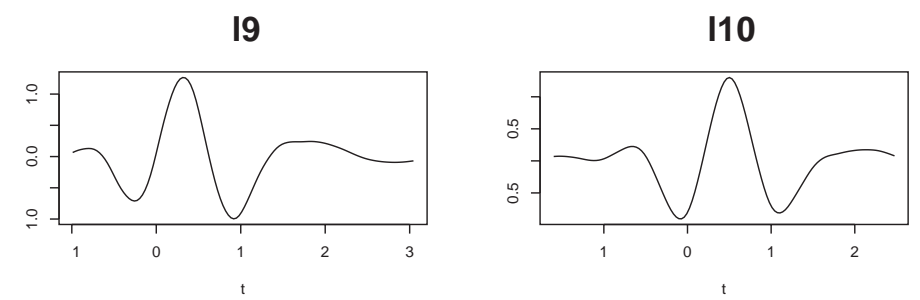

Figura 2.3: Ondaletas Daublets Menos Assimétricas. 
iii) $\cap_{j} V_{j}=\{0\}$.

iv) $g(t) \in V_{j}$ se e somente se $g(2 t) \in V_{j+1}$.

v) $V_{j+1}=V_{j} \oplus W_{j}, W_{j} \perp V_{j}$.

Considere uma função $g$ de quadrado integrável e que desejamos obter aproximações desta função em vários níveis de resolução. Cada um dos subespaços $V_{i}$ conterá funções aproximantes e a aproximação com menor distância é dada pela projeção ortogonal de $g$ em cada um desses subespaços. Assim, como $V_{i} \subset V_{i+1}$, se passarmos a projeção de $V_{i}$ para $V_{i+1}$ adicionamos detalhes à aproximação da função. Além disso da propriedade (v), verifica-se que os detalhes estão em um espaço ortogonal ao anterior. Pode-se mostrar também, que o sistema $\left\{\psi_{j, k}, k \in \mathbb{Z}\right\}$ forma uma base ortogonal para $W_{j}$ e $\left\{\phi_{j, k}, k \in \mathbb{Z}\right\}$ forma uma base ortogonal para $V_{j}$.

Assim, podemos verificar a seguinte relação:

$$
L_{2}(\mathbb{R})=\bigoplus_{j \in \mathbb{Z}} W_{j}=V_{0} \bigoplus \bigoplus_{j \geq 0} W_{j}=V_{j 0} \bigoplus \bigoplus_{j \geq j_{0}} W_{j}
$$

onde $j_{0}$ é qualquer número inteiro. Note que dependendo da escolha de $j_{0}$, podemos separar a função em um termo da resolução e outro de detalhes.

\subsubsection{A transformada de Ondaletas}

Grossman e Morlet $(1984,1985)$ foram os pioneiros no estudo da transformada contínua de ondaletas. A transformada contínua de ondaletas de um função $f$ de quadrado integrável é dada pelo produto escalar

$$
W_{\psi}(a, b)=<f ; \psi_{a, b}>=|a|^{-1 / 2} \int_{-\infty}^{\infty} f(t) \psi_{a, b}\left(\frac{t-b}{a}\right) d t
$$

com $a, b \in \mathbb{R}, a \neq 0$. No entanto, na prática, a maioria das séries temporais e processos estocásticos apresentam-se na forma discreta $X=\left(X_{0}, X_{1}, \ldots, X_{T}\right)$.

Assumindo $T=2^{M}$ e $M>0$, a transformada discreta de ondaletas de $X$, com respeito à geratriz $\psi$ é definida por

$$
d_{j, k}=\sum_{t=0}^{T-1} X_{t} \psi_{j, k}(t / T)
$$


a qual é calculada para $j=0,1, \ldots, M-1$ e $k=0,1, \ldots, 2^{j}-1$. No entanto, só teremos $T-1$ coeficientes, dessa forma, assumiremos que $d_{-1,0}$ é o coeficiente faltante. Assim, temos que

$$
X_{t}=d_{-1,0} \phi(t)+\sum_{j=0}^{M-1} \sum_{k=0}^{2^{j}-1} d_{j, k} \psi_{j, k}(t),
$$

onde $\phi(t)$ é a função escala ou ondaleta-pai.

Na prática, a transformada discreta de ondaletas não é calculada utilizando a definição anterior, mas utilizando-se o algoritmo piramidal, que baseia-se na construção de ondaletas utilizando os filtros passa-alta $\left(h_{k}\right)$ e passa-baixa $\left(l_{k}\right)$, como descrito em Mallat (1989).

\subsection{Análise de Ondaletas}

\subsubsection{Limiarização}

A utilização de limiarização (thresholding ou shrinkage) tem por objetivo a redução ou remoção de ruídos de um sinal. Os principais estudos realizados nesta área foram feitos por Donoho e Johnstone et al. (1994, 1995a, 1995b, 1998). Em outras palavras, a limiarização consiste em um processo de suavização não-paramétrico utilizando as funções de ondaletas. Assim, se assumimos que um sinal é da forma

$$
x_{t}=f(t)+\epsilon_{t}
$$

estamos interessados na estimação da função $f(t)$. Existem muitos outros métodos nãoparamétricos para a suavização, tais como o estimador de Nadaraya-Watson, séries de Fourier e splines. No entanto, como foi visto anteriormente, as ondaletas são adequadas para aproximar funções de espaços com suavidade não-homogênea, como espaços de Besov. Dessa forma, a adaptatividade das ondaletas nestes espaços é superior em relação a adaptatividade de outros métodos tradicionais.

A limiarização consiste na redução ou anulação de determinados coeficientes de ondaletas, utilizando-se determinados critérios. Em geral, para a redução de ruídos, utiliza-se o Soft Threshold, definido por

$$
\delta_{\lambda}^{S}=\left\{\begin{array}{cl}
0 & ,|x| \leq \lambda \\
\operatorname{sign}(x)(|x|-\lambda) & ,|x|>\lambda
\end{array}\right.
$$


Para remoção de ruídos utiliza-se o Hard Threshold definido por

$$
\delta_{\lambda}^{H}= \begin{cases}0 & ,|x| \leq \lambda \\ x & ,|x|>\lambda\end{cases}
$$

Existem diversos critérios para a escolha do limiar $\lambda$, tais como o universal (Donoho e Jonhstone, 1994), SureShrink (Donoho e Johnstone, 1995), cross-validation (Efron e Tibshirani, 1993), etc. Devido à sua simplicidade, um dos critérios mais utilizado é o limiar universal definido por $\lambda_{T}=\sigma \sqrt{2 \log T}$, e que não depende da escala. O parâmetro que mede o nível de ruído $\sigma$ deve ser estimado a partir dos dados. A Figura 2.4 mostra uma ilustração da limiarização universal utilizando diferentes ondaletas Daublets de Dupla Fase Extrema, onde a curva original é uma densidade gaussiana adicionada de um ruído $\left(g(t)+\epsilon_{t}\right)$.

d1

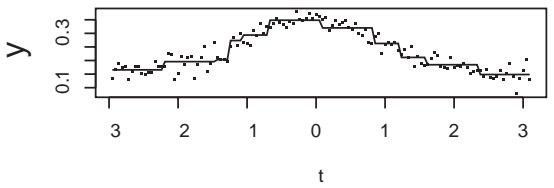

d3

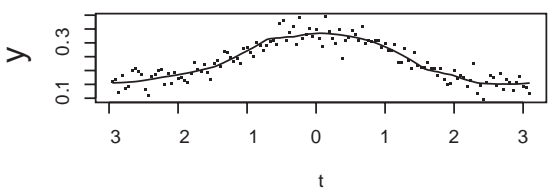

d5

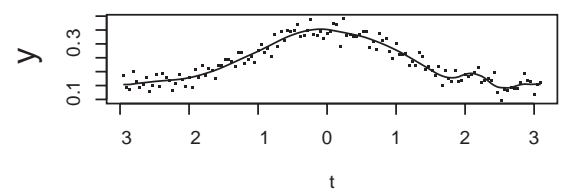

d7

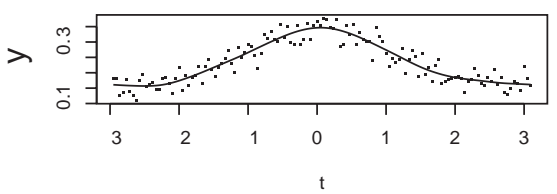

d2

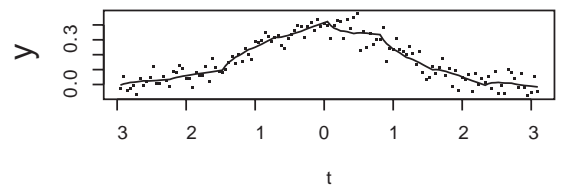

d4

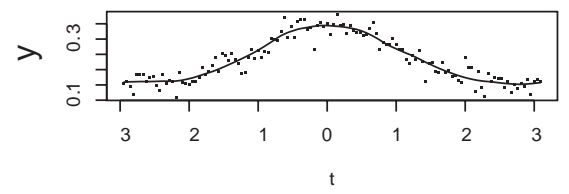

d6

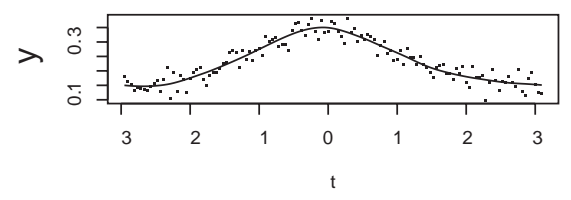

d8

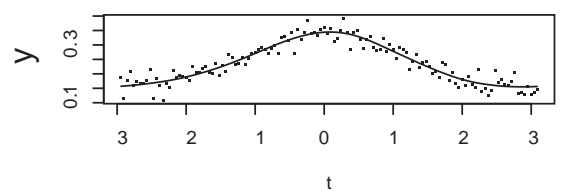

Figura 2.4: Suavização utilizando limiarização universal e diferentes funções de ondaletas. 


\subsubsection{Ondaletas e Processos Estacionários}

Considere $y(t)$ um processo estacionário de segunda ordem, com função de autocorrelação $\gamma(s, t)$ dada por

$$
\gamma(t, s)=\operatorname{Cov}(y(t), y(s))=E[(y(t)-E[y(t)])(y(s)-E[y(s)])],
$$

com

$$
\int_{\mathbb{R}} \sqrt{\gamma(t, t)}\left|\psi_{j, k}(t)\right| d t<\infty
$$

A transformada discreta de ondaletas do processo $y(t)$ é um variável aletória da forma

$$
\left\{d_{j, k}, j, k \in \mathbb{Z}\right\}=\left\{\int_{\mathbb{R}} y(t) \psi_{j, k}(t) d t, j, k \in \mathbb{Z}\right\}
$$

Assim sendo, temos que a covariância entre as transformadas de ondaletas de $y(t)$ é dada por

$$
E\left[d_{j, k} d_{j^{\prime}, k^{\prime}}\right]=\iint_{\mathbb{R}^{\not}} \gamma(t, s) \psi_{j, k}(t) \psi_{j^{\prime}, k^{\prime}}(s) d t d s
$$

Teorema 2.2.1. (Vidakovic, 1999) Seja $\{y(t), t \in \mathbb{R}\}$ um processo estocástico estacionário de segunda ordem com $\gamma(t, s)$ limitada e contínua em $\mathbb{R}^{2}$. Considere $d_{j, k}$ definido para todo $j, k$. Então $\left\{d_{j, k}, k \in \mathbb{Z}\right\}$ é um processo fracamente estacionário se e somente se $y(t)$ é fracamente estacionário.

Observação: A condição de $\gamma(t, s)$ limitada pode ser relaxada se as bases de ondaletas são de suporte compacto.

Este teorema é útil, pois indica que determinadas técnicas e metodologias aplicadas à séries estacionárias no domínio do tempo, podem também ser utilizadas no domínio de ondaletas, permitindo uma melhor caracterização do processo estocástico, e até mesmo revelando novas propriedades que não são facilmente identificáveis no domínio do tempo.

Uma outra propriedade interessante da transformada de ondaletas é que ela pode ser utilizada para remover a autocorrelação de séries temporais estacionárias (whitening). Considere

$$
y(t)=\sum_{j=1}^{m-1} \sum_{k} d_{j, k} \psi_{j, k}(t)
$$


de forma que

$$
d_{j, k}=\int_{\mathbb{R}} y(t) \psi_{j, k}(t) d t
$$

Note que

$$
\begin{aligned}
E\left[d_{j, k} d_{j^{\prime}, k^{\prime}}\right] & =\iint_{\mathbb{R}^{\sharp}} y(t) y(s) \psi_{j, k}(t) \psi_{j^{\prime}, k^{\prime}}(s) d t d s \\
& =\int_{\mathbb{R}} \hat{\gamma}(\omega) \Psi\left(\omega / 2^{j}\right) \overline{\Psi\left(\omega / 2^{j}\right)} e^{-i \omega k 2^{-j}+i \omega k^{\prime} 2^{-j^{\prime}}} 2^{-j / 2-j^{\prime} / 2}
\end{aligned}
$$

onde $\Psi$ e $\hat{\gamma}$ são as transformadas de Fourier de $\psi$ e $\gamma$, respectivamente.

Teorema 2.2.2. (Vidakovic, 1999) Seja uma base de ondaletas do tipo de Meyer, de forma que tanto $\Psi$ e $\hat{\gamma}$ pertencem à $\mathbb{C}^{p}, p>1$. Então, temos que os coeficientes $d_{j, k}$ e $d_{j^{\prime}, k^{\prime}}$

i) são não correlacionados se $\left|j-j^{\prime}\right|>1$;

ii) possuem baixa correlação arbitrária se $\left|j-j^{\prime}\right|=1$;

iii) possuem correlação da ordem de $O\left(\left|k-k^{\prime}\right|^{p}\right.$ se $j=j^{\prime}$.

Portanto, note que para uma escala fixada, a série formada pelas respectivas transformadas de ondaletas é estacionária e assintóticamente não-correlacionada, se o processo estocástico original for estacionário. Esta é propriedade é extremamente útil para testes de hipóteses baseados em bootstrap, pois as permutações e aleatorizações requerem dados não correlacionados.

\subsubsection{Espectro de Ondaletas}

Analogamente ao caso da representação espectral localizada na freqüência, no caso da decomposição em ondaletas temos uma representação tempo-escala. Baseando-se no conceito de processos localmente estacionários, Chiann e Morettin (1998) e von Sachs, Nason e Kroisandt $(1996,1997)$ introduziram o espectro evolucionário de ondaletas, o qual quantifica a energia da decomposição da variância-covariância de um processo estocástico em diferentes escalas e localizações ao longo do tempo. Um estimador para este espectro é o periodograma de ondaletas (escalograma), definido como o quadrado dos coeficientes da decomposição discreta de ondaletas de um processo estocástico. 
Considere um processo discreto estacionário de segunda ordem $y_{t}$ com função de covariância $\gamma_{h}=\operatorname{cov}\left(y_{t}, y_{t+h}\right)$. Assuma que o processo $y_{t}$ foi observado ao longo do tempo, de forma que temos $T=2^{m}$ observações.

Definição 2.2.1. (Chiann e Morettin, 1998)

Assuma que

$$
\sum_{t \in \mathbb{Z}}(1+|t|)\left|\gamma_{t}\right|<\infty
$$

O espectro de ondaletas de $y_{t}$ com respeito à ondaleta $\psi_{j, k}$ é dado por

$$
\eta_{j, k}=\sum_{u \in \mathbb{Z}} \gamma_{u} \Psi_{j, k}(u), j, k \in(Z)
$$

onde

$$
\Psi_{j, k}(u)=\sum_{t=0}^{\infty} \psi_{j, k}(t) \psi_{j, k}(t+|u|),
$$

é a função de autocorrelação de ondaletas.

Definição 2.2.2. O periodograma de ondaletas de um processo discreto estacionário de segunda ordem $y_{t}$ é dado por

$$
I_{j, k}=\left(\sum_{t=T-1}^{\infty} y_{t} \psi_{j, k}(t)\right)^{2} .
$$

Chiann e Morettin (1998) mostraram que o periodograma de ondaletas é um estimador assintóticamente não viesado para o espectro de ondaletas, no entanto, a variância do estimador não converge para zero quando $T$ cresce, de forma que não é consistente. Além disso, temos que $\operatorname{Var}\left[d_{j, k}\right] \rightarrow \eta_{j, k}$ quando $T \rightarrow \infty$. 


\section{Modelo Autoregressivo Vetorial}

Neste capítulo, serão apresentadas alguns conceitos e metodologias para a modelagem da conectividade cerebral. Apresentaremos o modelo econométrico autoregressivo vetorial (VAR), o conceito de causalidade de Granger e como estes podem ser úteis para a detecção do fluxo de informação entre módulos neuronais.

\subsection{Modelo Autoregressivo Vetorial}

Um modelo autoregressivo vetorial (VAR) de ordem p é definido por (Lütkepohl, 1993):

$$
x_{t}=u+\mathbf{A}_{1} x_{t-1}+\ldots+\mathbf{A}_{p} x_{t-p}+\epsilon_{t},
$$

onde $x_{t}=\left(x_{1 t}, \ldots, x_{s t}\right)^{\prime}$ é um vetor aleatório de dimensões $s \times 1, \mathbf{A}_{\mathbf{i}}$ 's são matrizes $s \times s$ de coeficientes autoregressivos, $u=\left(u_{1}, \ldots, u_{s}\right)^{\prime}$ é um vetor de interceptos e $\epsilon_{t}=\left(\epsilon_{1 t}, \ldots, \epsilon_{s t}\right)^{\prime}$ é um vetor de erros aleatórios ruidos brancos com matriz de covariância $\Sigma$. A matriz de coeficientes autoregressivos $\mathbf{A}_{\mathbf{i}}$ 's e a matriz de covariância $\boldsymbol{\Sigma}$ são da forma:

$$
\mathbf{A}_{i}=\left(\begin{array}{cccc}
a_{11}^{(i)} & a_{12}^{(i)} & \ldots & a_{1 s}^{(i)} \\
a_{21}^{(i)} & a_{22}^{(i)} & \ldots & a_{2 s}^{(i)} \\
\vdots & \vdots & \ddots & \vdots \\
a_{s 1}^{(i)} & a_{s 2}^{(i)} & \ldots & a_{s s}^{(i)}
\end{array}\right)
$$




$$
\boldsymbol{\Sigma}=\left(\begin{array}{cccc}
\sigma_{1}^{2} & \sigma_{12} & \ldots & \sigma_{1 s} \\
\sigma_{21} & \sigma_{2}^{2} & \ldots & \sigma_{2 s} \\
\vdots & \vdots & \ddots & \vdots \\
\sigma_{s 1} & \sigma_{s 2} & \ldots & \sigma_{s}^{2}
\end{array}\right)
$$

e note que estamos considerando a notação $\sigma_{1}^{2}=\sigma_{11}$.

Dizemos que um processo VAR satisfaz a condição de estabilidade se

$$
\operatorname{det}\left(\mathbf{I}_{s}-\sum_{i=1}^{p} \mathbf{A}_{1} z^{i}\right) \neq 0
$$

para qualquer valor de $z$ tal que $|z| \leq 1$. Assumindo que a condição de estabilidade está satisfeita, o processo VAR possui uma representação em termos de médias móveis (MA) dada por

$$
x_{t}=u+\epsilon_{t}+\sum_{i=1}^{\infty} \mathbf{C}_{i} \epsilon_{t-i},
$$

onde $x_{t}$ é representado em termos de valores passados e presentes dos erros $\epsilon_{t}$. Como $\epsilon_{t}$ é ruído branco, é imediato que se um processo $\operatorname{VAR}(p)$ satisfaz a condição de estabilidade, então este é um processo estacionário.

\subsubsection{Estimação dos Parâmetros}

Assuma que temos observações da série temporal multivariada $x_{t}$ s-dimensional em $T$ instantes de tempo, gerada por um processo $\operatorname{VAR}(p)$. Desejamos estimar as matrizes de coeficientes autoregressivos $\mathbf{A}_{\mathbf{i}}$ 's o vetor de interceptos $u$ e a matriz de covariâncias dos erros $\Sigma$.

Considere as seguintes matrizes

$$
\mathbf{X}_{t}=\left(\begin{array}{cccc}
x_{1,(p+1)} & x_{2,(p+1)} & \ldots & x_{s,(p+1)} \\
x_{1,(p+2)} & x_{2,(p+2)} & \ldots & x_{s,(p+2)} \\
\vdots & \vdots & \ddots & \vdots \\
x_{1, T} & x_{2, T} & \ldots & x_{s, T}
\end{array}\right)
$$




$$
\begin{gathered}
\mathbf{X}_{t-l}=\left(\begin{array}{cccc}
x_{1,(p-l+1)} & x_{2,(p-l+1)} & \ldots & x_{s,(p-l+1)} \\
x_{1,(p-l+2)} & x_{2,(p-l+2)} & \ldots & x_{s,(p-l+2)} \\
\vdots & \vdots & \ddots & \vdots \\
x_{1,(T-l)} & x_{2,(T-l)} & \ldots & x_{s,(T-l)}
\end{array}\right), l=1,2, \ldots, p \\
\mathbf{B}=\left(\begin{array}{llll}
u & A_{1} & \ldots & A_{p}
\end{array}\right) .
\end{gathered}
$$

Definição 3.1.1. O operador vec é tal que dados os vetores coluna $a_{i}$ de ordem $(k \times 1)$, $i=1,2, \ldots N$, temos

$$
\operatorname{vec}\left(\begin{array}{llll}
a_{1} & a_{2} & \ldots & a_{N}
\end{array}\right)=\left(\begin{array}{c}
a_{1} \\
a_{2} \\
\vdots \\
a_{N}
\end{array}\right)
$$

ou seja, o operador vec transforma uma matriz $k \times N$ em um vetor $N k \times 1$.

Na forma matricial, o modelo fica dado por

$$
Y=\mathbf{Z} \beta+\eta
$$

onde

$$
\begin{array}{r}
Y=\operatorname{vec}\left(\mathbf{X}_{t}\right), \\
\beta=\operatorname{vec}(\mathbf{B}), \\
\mathbf{Z}=\left(\mathbf{X}_{t-1}, \ldots, \mathbf{X}_{t-p}\right)
\end{array}
$$

Dessa forma, o estimador de mínimos quadrados de vec(B) é dado por

$$
\hat{\beta}=\left(\mathbf{I}_{s} \otimes\left(\mathbf{Z}^{\prime} \mathbf{Z}\right)^{-1} \mathbf{Z}^{\prime}\right) Y
$$

Além disso, é possível verificar que o estimador multivariado de mínimos quadrados é idêntico ao que seria obtido se utilizássemos o estimador de mínimos quadrados ordinários separadamente para cada uma das $s$ equações, o que é computacionalmente mais adequado. A estimativa da matriz de covariância $(\hat{\Sigma})$ pode ser facilmente obtida utilizando a covariância amostral dos resíduos obtidos de cada uma dessas equações. 
Proposição 3.1.1. (Lütkepohl, 1993) Seja $\mathbf{\Gamma}$ o limite em probabilidade de $\mathbf{Z}^{\prime} \mathbf{Z} / T$ quando $T \rightarrow \infty$. Então

$$
\sqrt{T}(\hat{\beta}-\beta) \stackrel{D}{\rightarrow} N\left(0, \Sigma \otimes \Gamma^{-1}\right)
$$

quando $T \rightarrow \infty$. Portanto, $\hat{\beta}$ é consistente para $\beta$.

Granger (1969) definiu um conceito de "causalidade" através da previsibilidade, baseandose no fato de que a causa não pode ser posterior a respectiva conseqüência ou efeito. Assim, se uma série temporal Granger-causa uma segunda, provavelmente os valores passados da primeira ajudam a prever o futuro da segunda. Em termos formais, considere $\mathscr{F}_{t}$ a sigmaálgebra contendo todas as informações relevantes até o periodo $t$. Considere $x_{t}\left(h \mid \mathscr{F}_{t}\right)$ o melhor preditor de horizonte $h$ do processo $x_{t}$, baseado nas informações contidas em $\mathscr{F}_{t}$. Seja $\Sigma\left(h \mid \mathscr{F}_{t}\right)$ o erro quadrático médio de previsão de horizonte $h$.

Definição 3.1.2. Um processo $y_{t}$ Granger-causa $x_{t}$ se $\Sigma\left(h \mid \mathscr{F}_{t}\right)<\Sigma\left(h \mid \mathscr{F}_{t}-\left\{y_{s} \mid s<t\right\}\right)$. E indicamos a causalidade por $y_{t} \stackrel{G}{\rightarrow} x_{t}$.

\subsubsection{Direcionalidade}

Primeiramente, note que considerando a definição de causalidade de Granger dada, a causalidade não é necessariamente recíproca. O fato de que $y_{t} \stackrel{G}{\rightarrow} x_{t}$ não implica que $x_{t} \stackrel{G}{\rightarrow} y_{t}$. Assim sendo temos três tipos de causalidade de Granger direcionadas:

- Autodependência: ocorre quando a série temporal Granger-causa a si própria, isto é, o passado da própria série ajuda na predição da mesma. Assim sendo, o passado traz informações que ajudam a explicar o futuro, e tal fato pode ser interpretado como uma reutilização da informação disponível. Alguns exemplos de séries com autodependência:

1) $x_{t}=\alpha x_{t-1}+\epsilon_{t}$

2) $x_{t}=\sum_{j=1}^{p} c_{j} x_{t-j}+\sum_{i=0}^{q} d_{i} \epsilon_{t-i}$,

3) $x_{t}=\sum_{j=1}^{p} c_{j} x_{t-j}+\sum_{i=0}^{q} d_{i} y_{t-i}+\epsilon_{t}$.

- Causalidade unidirecional: ocorre quando não existe reciprocidade, isto é, uma série Granger-causa a segunda, mas esta não Granger-causa a primeira. Note que esta propriedade pode ser interpretada como a existência de um único fluxo de informações. Exemplos de causalidade de Granger unidirecional são dados por: 
1) $x_{t}=\phi y_{t-1}+\epsilon_{t}$

2)

$$
\left(\begin{array}{l}
x_{t} \\
y_{t}
\end{array}\right)=\left(\begin{array}{c}
\sum_{j=1}^{p} a_{j} x_{t-j} \\
\sum_{j=1}^{s} b_{j} x_{t-j}
\end{array}\right)+\left(\begin{array}{c}
\sum_{i=1}^{q} c_{j} y_{t-i} \\
0
\end{array}\right)+\left(\begin{array}{c}
\epsilon_{t} \\
\xi_{t}
\end{array}\right) .
$$

- Causalidade bidirecional (feedback): ocorre quando existe reciprocidade na causalidade. Esta propriedade implica que existe uma forte relação de equilíbrio entre as duas séries, pois o passado de uma delas traz informações sobre o futuro da segunda e vice-versa. Um exemplo de causalidade de Granger bidirecional é um modelo VAR bivariado, dado por

$$
\left(\begin{array}{c}
x_{t} \\
y_{t}
\end{array}\right)=\left(\begin{array}{c}
\sum_{j=1}^{p} a_{j} x_{t-j} \\
\sum_{j=1}^{s} b_{j} x_{t-j}
\end{array}\right)+\left(\begin{array}{c}
\sum_{i=1}^{q} c_{j} y_{t-i} \\
\sum_{i=1}^{m} d_{j} y_{t-i}
\end{array}\right)+\left(\begin{array}{c}
\epsilon_{t} \\
\xi_{t}
\end{array}\right) .
$$

Além dos três tipos de causalidade de Granger direcionadas definidos anteriormente, podemos considerar relações não direcionadas. Exemplificando, considere o modelo VAR bivariado definido no exemplo anterior. Se a matriz de covariância dos erros $\xi_{t}$ e $\epsilon_{t}$ não for diagonal, dizemos que existe causalidade de Granger instantânea entre $x_{t}$ e $y_{t}$. A causalidade de Granger quantifica a simultaneidade entre duas séries e é recíproca, isto é, não possui direção.

\subsubsection{Testes de Hipóteses}

Como vimos, o modelo VAR utiliza os valores passados da série $s$-dimensional $x_{t}$ para gerar previsões. Assim, a causalidade de Granger está bem definida nesta classe de modelos.

Proposição 3.1.2. (Lütkepohl, 1993) Se $x_{t}$ é um processo VAR(p) que satisfaz a condição de estabilidade, e $a_{i j}^{(l)}$ é o elemento da i-ésima linha da j-ésima coluna da matriz de coeficientes autoregressivos de ordem $l, \mathbf{A}_{l}$, então a série $x_{j t}$ Granger causa a série $x_{i t}$ se e somente se $a_{i j}^{(l)} \neq 0$, para algum $l$.

Dessa forma, para testarmos a não-causalidade, podemos testar se os coeficientes autoregressivos são estatisticamente iguais a zero. Assim, suponha que desejamos testar 
combinações lineares dos parâmetros do vetor $\beta$. Em outras palavras, queremos realizar o seguinte teste de hipóteses:

$$
\begin{aligned}
& H_{0}: \mathbf{C} \beta=m \\
& H_{A}: \mathbf{C} \beta \neq m
\end{aligned}
$$

onde $\mathbf{C}$ é uma matriz de contrastes. O teste de Wald é dado por

$$
W=(\mathbf{C} \hat{\beta}-m)^{\prime}\left[\mathbf{C}\left(\left(Z^{\prime} Z\right)^{-1} \otimes \hat{\mathbf{\Sigma}}\right) \mathbf{C}^{\prime}\right]^{-1}(\mathbf{C} \hat{\beta}-m),
$$

onde $\mathrm{W}$ possui distribuição qui-quadrado com $\operatorname{posto}(\mathbf{C})$ graus de liberdade.

Assim, o teste para $\beta=0_{s(s+1)}$ pode ser feito tomando-se $\mathbf{C}=\mathbf{I}_{s(s+1)}$ e $m=0_{s(s+1)}$. Para avaliar a causalidade de Granger, o teste individual para cada um dos elementos de $\beta$ também pode ser facilmente realizado via contrastes.

\subsection{Conectividade Cerebral e Causalidade de Gran- ger}

A identificação de processsos corticais é um passo fundamental para um melhor entendimento dos processos neurocognitivos. Muitos estudos (D'Esposito et al. (2000) e Raichle et al.(2001) entre outros) indicam que a interação entre duas áreas cerebrais é uma das questões mais importantes a serem avaliadas, e ainda não se sabe com precisão, os mecanismos de interação entre módulos neuronais.

A conectividade em neurociências pode ser dividida em dois conceitos: conectividade funcional e efetiva. A conectividade funcional pode ser definida como "correlação da atividade cerebral de areas espacialmente remotas" e a efetiva como "influência de um sistema/modulo neuronal sobre outro"(Frackowiak et al., 2003). Note que a conectividade efetiva implica em uma conectividade funcional, mas a recíproca não é necessariamente válida. Um exemplo ilustrativo desta situação, pode ser um experimento com a apresentação de estímulos auditivos e visuais simultaneamente. É razoável esperar que a atividade neuronal no cortex auditivo e visual estejam correlacionados (conectividade funcional), no entanto, não há nenhuma razão para crer que exista uma interação entre essas regiões cerebrais (conectividade efetiva). No entanto, a simplicidade conceitual da conectividade funcional é extremamente atraente, principalmente em casos em que ativi- 
dade neuronal não medida diretamente, como no sinal do efeito BOLD.

Embora o caráter multivariado do modelo VAR e a identificação da causalidade de Granger estejam intuitivamente ligados a modelagem de uma conectividade efetiva, na prática, não há garantias de que somente uma nova face da conectividade funcional esteja sendo modelada. Basta considerar um exemplo onde um estímulo visual é sempre precedido de um auditivo. Provavelmente seria identificado um fluxo de informação da área auditiva para a área visual. Isso decorre do fato de que a causalidade de Granger não é capaz de discriminar uma influência real de um aumento no poder preditivo. Dessa forma, para a modelagem de uma conectividade efetiva, são necessárias algumas hipóteses sobre as vias anatômicas.

A maior parte dos estudos de conectividade funcional utiliza o coeficiente de correlação linear de Pearson dos sinais como medida de conectividade entre as áreas cerebrais (Biswal (1995) e Hampson (2002)). Em estudos de EEG, muitas vezes são utilizados métodos de análise espectral como função de coerência e espectro de fase. No entanto, utilizando estes métodos não é possível identificar a direção do fluxo de informação, mas apenas a simultaneidade ou sincronismo entre duas séries.

Assim, de forma a permitir a identificação do fluxo de informação, a modelagem através de equações estruturais (SEM) e mapas de caminhos tornou-se uma metodologia freqüentemente utilizada. Büchel e Friston (1997), através da SEM e RMf, modelaram as interações occipito-parieto-frontal envolvidas em tarefas que exigem atenção visual. McIntosh (1999) utilizou a mesma técnica para a modelagem de mecanismos envolvendo a memória. No entanto, são necessárias informações à priori da estrutura e direcionalidade da conectividade, para a modelagem de equações estruturais. O fluxo de informação é apenas quantificado e não identificado.

Mais tarde Baccalá e Sameshima (2001) introduziram o conceito de coerência parcial direcionada, um método de identificação da direção do fluxo de informações em EEG, utilizando conceitos de causalidade de Granger no domínio da freqüência e modelos VAR. Goebel et al. (2003) e Harrison (2003) introduziram o conceito de causalidade de Granger no contexto de RMf, utilizando o modelo VAR em experimentos visando a identificação de interações corticais em tarefas visual-motoras.

Atualmente, a causalidade de Granger em RMf vêm se tornando um conceito essencial para a modelagem das interações corticais (Valdes-Sosa (2004), Valdes-Sosa et al. (2005), Sato et al. (2006a), Sato et al. (2006b)). Como foi visto, a causalidade de Granger está 
bem especificada para o modelo $\operatorname{VAR}(\mathrm{p})$, e testes de causalidade podem ser efetuados de forma simples nesta classe de modelos. Além disso, alguns estudos sugerem a utilização da causalidade de Granger conjuntamente com a teoria dos grafos, de forma a obter ferramentas para uma análise global de conectividade (Dahlhaus et al. (1999) e Eichler (2005)).

Considere uma série temporal $s$-dimensional relativas ao sinal BOLD de $s$ áreas cerebrais. Sabemos que o sinal BOLD local está intimamente ligado com a atividade neuronal da região, de forma que a identificação do fluxo de informações entre áreas cerebrais pode ser avaliado através de um modelo VAR. Dessa forma, consideramos que se um sinal BOLD de uma área cerebral Granger causa o sinal BOLD de uma segunda, então há fluxo de informações da primeira região para a segunda.

Considere como o exemplo ilustrativo o seguinte modelo VAR:

$$
\left\{\begin{array}{l}
x_{1, t}=200+0.8 x_{1,(t-1)}-0.2 x_{2,(t-1)}+\epsilon_{1, t} \\
x_{2, t}=170-0.3 x_{1,(t-1)}+0.5 x_{2,(t-1)}+\epsilon_{2, t} \\
x_{3, t}=150+0.4 x_{1,(t-1)}-0.6 x_{2,(t-1)}+0.4 x_{3,(t-1)}+\epsilon_{3, t}
\end{array}\right.
$$

ou na forma matricial

$$
x_{t}=u+\mathbf{A}_{1} x_{t-1}+\epsilon_{t},
$$

onde $x_{t}=\left(x_{1 t}, x_{2 t}, x_{3 t}\right)^{\prime}, u=(200,170,150)^{\prime} \mathrm{e}$

$$
\mathbf{A}_{1}=\left(\begin{array}{ccc}
0.8 & -0.2 & 0 \\
-0.3 & 0.5 & 0 \\
0.4 & -0.6 & 0.4
\end{array}\right)
$$

Dessa forma, utilizando o conceito de causalidade de Granger, podemos construir o diagrama de fluxo de informações mostrado na Figura 3.1.

Uma possível interpretação para os coeficientes autoregressivos é a de que quanto maior seu módulo, maior a intensidade do fluxo de informações das áreas correspondentes. Além disso, coeficientes positivos indicam que a informação enviada de uma área à outra é de co-ativação, e coeficientes negativos refletem uma diferença de fase ou um possível efeito de inibição. No entanto, devido a limitações computacionais e também à problemas de superparametrização, não é possível a inclusão de todas os nós de interesse no modelo, e portanto, as interpretações sobre conectividades excitatórias ou inibitórias 


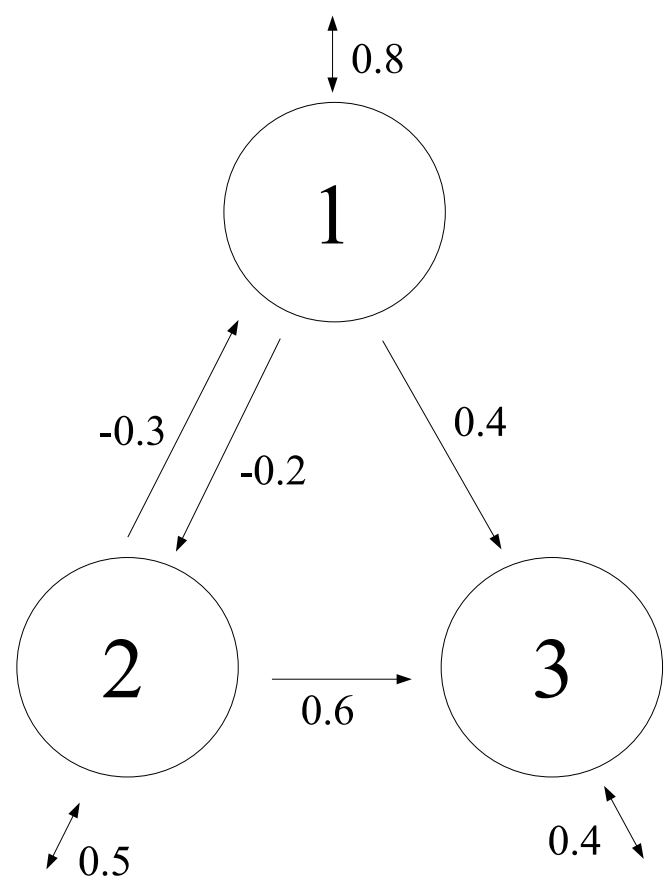

Figura 3.1: Diagrama de fluxo de informações.

estão apenas em um nível conjectural.

Além disso, é importante ressaltar que a identificação de conectividades entre áreas cerebrais distintas é absolutamente dependente do intervalo de amostragem dos dados. Assim, dado o fato que o intervalo de amostragem em experimentos de RMf (TR) é extremamente alto (1 a 4 segundos) e as interações neuronais se dão na ordem de milisegundos, é evidente que os fluxos de informação com duração inferior à taxa de amostragem não serão identificados. Em outras palavras, devido à limitada resolução temporal dos dados coletados em RMf, são apenas observados dados agregados. Assim, a maior parte das relações entre as séries temporais estão na causalidade de Granger instantânea, que como foi visto, não fornece informações a respeito da direção do fluxo de informações. No entanto, devido ao atraso da resposta hemodinâmica, muitos fenômenos de conectividade de longo prazo (baixas freqüências) ainda podem ser detectados utilizando-se os modelos VAR. Abler et al. (2006) mostrou a consistência das estimativas do modelo VAR para experimentos motores, utilizando uma amostra de 11 indivíduos.

No entanto, a modelagem VAR para dados obtidos em experimentos neurocognitivos baseados em RMf possui alguns obstáculos. Para muitos paradigmas que envolvem 
seqüências de estímulos ao longo do tempo, a suposição de estacionaridade não é válida. Focando esta deficiência, sugerimos a extensão localmente estacionária para o modelo VAR, tema do próximo capítulo. 


\section{Modelo Autoregressivo Vetorial Variante no Tempo}

Neste capítulo, será apresentada uma nova metodologia para a modelagem da conectividade cerebral variante no tempo, baseada no conceito de processos localmente estacionários (Dahlhaus et al., 1999). Este capítulo é essencialmente teórico, de forma a definir e caracterizar matematicamente a metodologia proposta. O modelo é baseado em modelos autoregressivos vetoriais com coeficientes variando no tempo. Apresentaremos o modelo, procedimentos para estimação e propriedades assintóticas.

\subsection{Modelo Autoregressivo Multivariado Dinâmico}

Seja $x_{t}$ uma série temporal multivariada $s$-dimensional $x_{t, T}=\left(x_{1 t, T}, x_{2 t, T}, \ldots, x_{s t, T}\right)^{\prime}$ com $T$ realizações (observações). O modelo DVAR (dynamic vector autoregression) é definido por

$$
\begin{aligned}
& x_{t, T}=u(t / T)+\mathbf{A}_{1}(t / T) x_{t-1, T}+\mathbf{A}_{2}(t / T) x_{t-2, T}+\ldots+\mathbf{A}_{p}(t / T) x_{t-p, T}+\epsilon_{t, T}, \\
& \operatorname{Var}\left(\epsilon_{t, T}\right)=\Sigma(t / T)=\left(\begin{array}{cccc}
\sigma_{1}^{2}(t / T) & \sigma_{12}(t / T) & \ldots & \sigma_{1 s}(t / T) \\
\sigma_{21}(t / T) & \sigma_{2}^{2}(t / T) & \ldots & \sigma_{2 s}(t / T) \\
\vdots & \vdots & \ddots & \vdots \\
\sigma_{s 1}(t / T) & \sigma_{s 2}(t / T) & \ldots & \sigma_{s}^{2}(t / T)
\end{array}\right),
\end{aligned}
$$

onde $u(t / T)$ é um vetor de interceptos, e $\mathbf{A}_{l}(t / T)$ uma matriz de coeficientes, ambos funções do tempo, dados por 


$$
u(t / T)=\left(\begin{array}{c}
u_{1}(t / T) \\
u_{2}(t / T) \\
\vdots \\
u_{s}(t / T)
\end{array}\right), \quad \mathbf{A}_{l}(t / T)=\left(\begin{array}{cccc}
a_{11}^{(l)}(t / T) & a_{12}^{(l)}(t / T) & \ldots & a_{1 s}^{(l)}(t / T) \\
a_{21}^{(l)}(t / T) & a_{22}^{(l)}(t / T) & \ldots & a_{2 s}^{(l)}(t / T) \\
\vdots & \vdots & \ddots & \vdots \\
a_{s 1}^{(l)}(t / T) & a_{s 2}^{(l)}(t / T) & \ldots & a_{s s}^{(l)}(t / T)
\end{array}\right),
$$

$l=1, \ldots, p$.

Note que o intercepto, parâmetros autoregressivos e matriz de autocovariância dos erros são funções do tempo, mas são adequadamente re-escalados para funções de suporte compacto no intervalo $[0,1]$.

Diremos que um processo DVAR satisfaz a condição de estabilidade local, se para cada instante de tempo $t$,

$$
\operatorname{det}\left(\mathbf{I}_{s}-\sum_{i=1}^{p} \mathbf{A}_{1}(t / T) z^{i}\right) \neq 0
$$

para qualquer valor de $z$ tal que $|z| \leq 1$. Se a condição de estabilidade local está satisfeita, então o processo DVAR possui uma representação $\mathrm{MA}(\infty)$ dada por

$$
\begin{array}{r}
x_{t, T}=u(t / T)+\epsilon_{t, T}+\sum_{1}^{\infty} \mathbf{C}_{i}(t / T) \epsilon_{t-i, T}, \\
\sup _{t} \sum_{1}^{\infty}\left|\mathbf{C}_{i}(t / T)\right|<\infty .
\end{array}
$$

Como $\epsilon_{t}$ é ruído branco, é imediato que se um processo $\operatorname{DVAR}(p)$ satisfaz a condição de estabilidade local, então este é um processo localmente estacionário (Dahlhaus, 2000).

Definição 4.1.1. (Dahlhaus, 2000) Uma seqüência de processos estocásticos gaussianos multivariados $x_{t, T}=\left(x_{t, T}^{(1)}, \ldots, x_{t, T}^{(s)}\right)^{\prime}$ com $t=1, \ldots, T$ é um processo localmente estacionário com matriz função de transferência $\mathbf{A}_{t, T}^{0}(\lambda)$ e vetor função média $\mu(t / T)$ se existe a representação

$$
x_{t, T}=\mu\left(\frac{t}{T}\right)+\int_{-\pi}^{\pi} \exp (i \lambda t) \mathbf{A}_{t, T}^{0}(\lambda) d \xi(\lambda),
$$

com as seguintes propriedades:

i) $\xi(\lambda)$ é um vetor de processos ortonormais complexos em $[-\pi, \pi] \operatorname{com} \overline{\xi_{a}(\lambda)}=\xi_{a}(-\lambda)$, $E\left[\xi_{a}(\lambda)\right]=0 e$

$$
E\left[d \xi_{a}(\lambda) d \xi_{b}(\lambda)\right]=\delta_{a b} \eta(\lambda+\mu) d \lambda d \mu
$$


onde $\eta(\lambda)=\sum_{j=-\infty}^{\infty} \delta(\lambda+2 \pi j)$ é a extensão de período $2 \pi$ da função delta de Dirac;

ii) Existe uma constante $K$ e uma matriz de funções $2 \pi$ periódica $\mathbf{A}:[0,1] \times \mathbb{R} \rightarrow \mathbb{C}^{s \times s}$ $\operatorname{com} \overline{\mathbf{A}(u, \lambda)}=\mathbf{A}(u,-\lambda) e$

$$
\sup _{t, \lambda}\left|\mathbf{A}_{t, T}^{0}(\lambda)_{a b}-\mathbf{A}(t / T, \lambda)_{a b}\right| \leq K T^{-1}
$$

para todo $a, b=1, \ldots, s$ e $T \in \mathbb{N}$. $\mathbf{A}(u, \lambda)$ e $\mu(u)$ são funções contínuas em $u$.

Dizemos que $f(u, \lambda):=\mathbf{A}(u, \lambda) \overline{\mathbf{A}(u, \lambda)^{\prime}}$ é a matriz densidade espectral evolucionária do processo.

Para facilitar a notação e evitar equívocos, deste ponto em diante denotamos $x_{t} \equiv x_{t, T}$ e $f(t) \equiv f(t / T)$, onde $x_{t, T}$ é um processo estocástico multivariado e $f(t / T)$ é uma função de suporte compacto em $[0,1]$. Considere também, por simplicidade de notação, que $\psi_{-1,0}(x)=\phi_{0,0}(x)$ de forma que o coeficiente $\alpha_{0,0}=\beta_{-1,0}$. Dessa forma, a expansão em ondaletas de uma função $f(x) \in L^{2}$ é dada por

$$
f(x)=\sum_{j=-1}^{\infty} \sum_{k=0}^{\infty} \beta_{j, k} \psi_{j, k}(x) .
$$

Note que os coeficientes do modelo DVAR são funções do tempo, e portanto construímos uma generalização do modelo VAR. Considerando $x_{t}$ um processo multivariado $s$ dimensional, as expansões em ondaletas das funções no vetor $u(t)$ e nas matrizes $\mathbf{A}_{l}(t)$ para $l=1, \ldots, p$, temos

$$
\begin{gathered}
u(t / T)=\sum_{j=-1}^{\infty} \sum_{k=0}^{\infty} u_{j, k} \psi_{j, k}(t), \\
\mathbf{A}_{l}(t / T)=\sum_{j=-1}^{\infty} \sum_{k=0}^{\infty} \mathbf{A}_{j, k}^{(l)} \psi_{j, k}(t) x_{t-l},
\end{gathered}
$$

onde $u_{j, k}$ e $\mathbf{A}_{j, k}^{(l)}(l=1, \ldots, p, j=1,2, \ldots$ e $k=1,2, \ldots)$ são um vetor $(s \times 1)$ e matrizes $(s \times s)$ contendo os coeficientes da expansão de ondaletas.

Assim sendo, o modelo autoregressivo multivariado com coeficientes variando no tempo 
pode ser representado como

$$
\begin{aligned}
x_{t}= & \sum_{j=-1}^{\infty} \sum_{k=0}^{\infty} u_{j, k} \psi_{j, k}(t)+\sum_{l=1}^{p} \sum_{j=-1}^{\infty} \sum_{k=0}^{\infty} \mathbf{A}_{j, k}^{(l)} \psi_{j, k}(t) x_{t-l}+\epsilon_{t}, \\
= & \sum_{j=-1}^{J} \sum_{k=0}^{2^{j}-1} u_{j, k} \psi_{j, k}(t)+\sum_{l=1}^{p} \sum_{j=-1}^{J} \sum_{k=0}^{2^{j}-1} \mathbf{A}_{j, k}^{(l)} \psi_{j, k}(t) x_{t-l}+ \\
& +\sum_{j>J} \sum_{k=0}^{\infty} u_{j, k} \psi_{j, k}(t)+\sum_{l=1}^{p} \sum_{j>J} \sum_{k=0}^{\infty} \mathbf{A}_{j, k}^{(l)} \psi_{j, k}(t) x_{t-l}+\epsilon_{t}, \\
= & \sum_{j=-1}^{J} \sum_{k=0}^{2^{j}-1} u_{j, k} \psi_{j, k}(t)+\sum_{l=1}^{p} \sum_{j=-1}^{J} \sum_{k=0}^{2^{j}-1} \mathbf{A}_{j, k}^{(l)} \psi_{j, k}(t) x_{t-l}+\nu_{t},
\end{aligned}
$$

onde

$$
\nu_{t}=\sum_{j>J} \sum_{k=0}^{\infty} u_{j, k} \psi_{j, k}(t)+\sum_{l=1}^{p} \sum_{j>J} \sum_{k=0}^{\infty} \mathbf{A}_{j, k}^{(l)} \psi_{j, k}(t) x_{t-l}+\epsilon_{t}=\eta_{t}+\epsilon_{t},
$$

Note que $\eta_{t}$ é termo de erro advindo do truncamento da expansão em ondaletas em uma escala $J=J(T)$. Quanto maior o valor de $J$, menor será o erro de aproximação $\eta_{t}=o(T)$.

Dessa forma, dada uma realização (trajetória) da variável $X_{t}$ em tempo discreto, desejamos estimar as funções $u(t), \mathbf{A}_{l}(t)$ para $l=1, \ldots, p$ e $\boldsymbol{\Sigma}(t)$ estimando os coeficientes da expansão de ondaletas. Note que neste caso, o número de parâmetros a serem estimados depende da dimensão $s$ da variável $x_{t}$ e do parâmetro de truncamento (resolução) $J$. É fácil verificar que, em geral, teremos muitos parâmetros a serem estimados, de forma que procedimentos como a estimação através de máxima verossimilhança serão computacionalmente intensivos e até mesmo não implementáveis na prática. Além disso, note que os erros $\nu_{t}$ dependem dos valores de $x_{t}$ e conseqüentemente são autocorrelacionados, dificultando ainda mais o cálculo da verossimilhança. Portanto, sugerimos um estimador baseado no método de mínimos quadrados generalizados de forma a reduzir o custo computacional.

\subsubsection{Estimação dos Parâmetros}

Primeiramente, suponha que a matriz de covariâncias $\boldsymbol{\Sigma}(t)$ seja conhecida. O modelo cujos parâmetros deverão ser estimados é

$$
x_{t}=\sum_{j=-1}^{J} \sum_{k=0}^{2^{j}-1} u_{j, k} \psi_{j, k}(t)+\sum_{l=1}^{p} \sum_{j=-1}^{J} \sum_{k=0}^{2^{j}-1} \mathbf{A}_{j, k}^{(l)} \psi_{j, k}(t) x_{t-l}+\nu_{t},
$$


onde $x_{t}$ é uma variável aleatória $s$ dimensional, $u_{j, k}$ é um vetor e $\mathbf{A}_{j, k}^{(l)}$ são matrizes de coeficientes.

Assumindo o truncamento na resolução $J=J(T)$, o modelo pode ser escrito na seguinte forma matricial.

Definição 4.1.2. Um modelo DVAR aproximado via ondaletas é dado por

$$
x_{t}=\boldsymbol{\Lambda}[Z \otimes \Psi(t)]^{\prime}+\nu_{t}
$$

onde $Z$ é um vetor linha $1 \times(s p+1)$ de valores passados da séries multivariada $x_{t} d a$ forma

$$
Z=\left[1, \quad x_{t-1}^{\prime}, \quad x_{t-2}^{\prime}, \ldots, \quad x_{t-p}^{\prime}\right]
$$

$\Psi(t)$ é um vetor linha $1 \times 2^{J}$ de funções de ondaletas da forma

$$
\Psi(t)=\left[\psi_{-1,0}(t), \quad \psi_{0,0}(t), \ldots, \quad \psi_{J, 2^{J}-1}(t)\right]
$$

e $\boldsymbol{\Lambda}$ é uma matriz $s \times(s p+1) 2^{J}$ com os respectivos coeficientes das expansões em ondaletas. Note que cada linha corresponde aos coeficientes de cada uma das s séries univariadas que compõem $x_{t}$.

Considere agora, que observamos uma realização de $x_{t}$ para $t=1,2, \ldots, T$. Dessa forma, dispondo os valores de $x_{t}$ na forma matricial, temos

$$
\begin{gathered}
\mathbf{X}_{\mathbf{t}}=\left(\begin{array}{cccc}
x_{1,(p+1)} & x_{2,(p+1)} & \ldots & x_{s,(p+1)} \\
x_{1,(p+2)} & x_{2,(p+2)} & \ldots & x_{s,(p+2)} \\
\vdots & \vdots & \ddots & \vdots \\
x_{1, T} & x_{2, T} & \ldots & x_{s, T}
\end{array}\right) \\
\mathbf{X}_{t-l}=\left(\begin{array}{cccc}
x_{1,(p-l+1)} & x_{2,(p-l+1)} & \ldots & x_{s,(p-l+1)} \\
x_{1,(p-l+2)} & x_{2,(p-l+2)} & \ldots & x_{s,(p-l+2)} \\
\vdots & \vdots & \ddots & \vdots \\
x_{1,(T-l)} & x_{2,(T-l)} & \ldots & x_{s,(T-l)}
\end{array}\right)
\end{gathered}
$$


$l=1, \ldots, p$ e também

$$
\boldsymbol{\Psi}=\left(\begin{array}{cccc}
\psi_{-1,0}(p+1) & \psi_{0,0}(p+1) & \ldots & \psi_{J, 2^{J}-1}(p+1) \\
\psi_{-1,0}(p+2) & \psi_{0,0}(p+2) & \ldots & \psi_{J, 2^{J}-1}(p+2) \\
\vdots & \vdots & \ddots & \vdots \\
\psi_{-1,0}(T) & \psi_{0,0}(T) & \ldots & \psi_{J, 2^{J}-1}(T)
\end{array}\right)
$$

Definição 4.1.3. O produto Kronecker Linha $\otimes^{L}$ é tal que dados os vetores linha $a_{i}$ e $b_{i}$, $i=1, \ldots, N$, temos

$$
\left(\begin{array}{c}
a_{1} \\
a_{2} \\
\vdots \\
a_{N}
\end{array}\right) \otimes^{L}\left(\begin{array}{c}
b_{1} \\
b_{2} \\
\vdots \\
b_{N}
\end{array}\right)=\left(\begin{array}{c}
a_{1} \otimes b_{1} \\
a_{2} \otimes b_{2} \\
\vdots \\
a_{N} \otimes b_{N}
\end{array}\right)
$$

Assim, considere as seguintes matrizes

$$
\begin{aligned}
& \mathbf{W}=\left[1_{N-p} \otimes^{L} \Psi, \quad \mathbf{X}_{t-1} \otimes^{L} \Psi, \ldots, \quad \mathbf{X}_{t-l} \otimes^{L} \Psi\right] \\
& \mathbf{M}=\mathbf{I}_{s} \otimes \mathbf{W},
\end{aligned}
$$

e o vetor

$$
Y=\operatorname{vec}\left(\mathbf{X}_{t}\right)
$$

Considere, agora, a matriz de covariâncias dos termos de erros aleatórios $\nu_{t}$ dada por

$$
\Sigma=\left(\begin{array}{cccc}
\operatorname{diag}\left[\sigma_{1}^{2}(t)\right] & \operatorname{diag}\left[\sigma_{12}(t)\right] & \ldots & \operatorname{diag}\left[\sigma_{1 s}(t)\right] \\
\operatorname{diag}\left[\sigma_{21}(t)\right] & \operatorname{diag}\left[\sigma_{2}^{2}(t)\right] & \ldots & \operatorname{diag}\left[\sigma_{2 s}(t)\right] \\
\vdots & \vdots & \ddots & \vdots \\
\operatorname{diag}\left[\sigma_{s 1}(t)\right] & \operatorname{diag}\left[\sigma_{s 2}(t)\right] & \ldots & \operatorname{diag}\left[\sigma_{s}^{2}(t)\right]
\end{array}\right)
$$

onde $\operatorname{diag}[f(t)]$ representa uma matriz diagonal, cujos elementos da diagonal principal são dados por $f(t)$ para $t=(p+1),(p+2), \ldots, T$.

$\mathrm{Na}$ notação matricial, considerando $\beta=\operatorname{vec}(\boldsymbol{\Lambda})$, o modelo fica dado por

$$
Y=\mathbf{M} \beta+\nu
$$

Lema 4.1.1. (Graybill, 1985) Assumindo $Y$ como o vetor resposta, M a matriz de planejamento de uma regressão e assumindo a matriz de variância dos erros conhecida $(\boldsymbol{\Sigma})$, 
o estimador de mínimos quadrados generalizados dos coeficientes de $\beta=v e c(\boldsymbol{\Lambda})$ é dado por

$$
\begin{aligned}
\hat{\beta} & =\left(\mathbf{M}^{\prime} \boldsymbol{\Sigma}^{-1} \mathbf{M}\right)^{-1} \mathbf{M}^{\prime} \boldsymbol{\Sigma}^{-1} Y \\
& =\left(\mathbf{D}^{\prime} \mathbf{D}\right)^{-1} \mathbf{D}^{\prime} V
\end{aligned}
$$

onde

$$
\begin{aligned}
& \mathbf{D}=\boldsymbol{\Sigma}^{-\frac{1}{2}} \mathbf{M}, \\
& V=\boldsymbol{\Sigma}^{-\frac{1}{2}} Y .
\end{aligned}
$$

Considere as seguintes condições:

(C1) Verifica-se que as bases de ondaletas induzem normas no espaço dos coeficientes, as quais são análogas a normas no espaço de Besov. Assim sendo, considere um espaço de Besov $B_{p, q}^{m}$, onde $m \leq 1$ é um coeficiente de suavidade das funções, e $(1 \leq p, q<\infty)$ são parâmetros de forma das funções. Para o modelo DVAR, assumiremos que todas as funções $u(t), \mathbf{A}_{i}(t)$ e $\boldsymbol{\Sigma}(t)$ são funções reais, limitadas pertencem ao seguinte conjunto:

$$
\mathscr{F}_{i}=\left\{f(x): f(x)=\sum_{j=-1}^{\infty} \sum_{k=0}^{\infty} \beta_{j, k}^{(i)} \psi_{j, k}(x) \mid\left\|\beta_{. .}^{(i)}\right\|_{m_{i}, p_{i}, q_{i}}<\infty\right\},
$$

onde

$$
\begin{aligned}
\left\|\beta_{. .}^{(i)}\right\|_{m_{i}, p_{i}, q_{i}} & =\left(\sum_{j \geq l}\left[2^{j s_{i} p_{i}} \sum_{k \in I_{j}}\left|\beta_{j, k}^{(i)}\right|_{i}^{p}\right]^{q_{i} / p_{i}}\right)^{1 / q_{i}}, \\
s_{i} & =m_{i}+1 / 2+1 / p_{i} \\
1 & <m_{i}+1 / 2-\min \left\{p_{i}, 2\right\} .
\end{aligned}
$$

(C2) $\phi$ e $\psi$ pertencem à $\mathscr{C}^{r}[0,1]$ e têm suporte compacto. Além disso $\int \phi(t) d t=1 \mathrm{e}$ $\int \psi(t) t^{k} d t=0$ para $0 \leq k \leq r$.

(C3) Existe um $\gamma \geq 0$ tal que $\left|\operatorname{cum}_{n}\left(\nu_{t}\right)\right| \leq C^{n}(n !)^{1+\gamma}$ para todo $n$ e $t$.

(C4) O processo $x_{t}$ é localmente estacionário, admitindo uma representação $\mathrm{MA}(\infty)$, isto é

$$
x_{t}=u(t)+\sum_{0}^{\infty} \mathbf{C}_{i}(t) \epsilon_{t-i}
$$


e

$$
\sup _{t}\left(\sum_{0}^{\infty}\left|C_{i}(t)\right|\right) \leq \infty
$$

(C5) $\left\|\Sigma^{-1}\right\|<\infty$.

Proposição 4.1.1. Considerando que as condições $(C 1)-(C 5)$ estão satisfeitas, que os erros são gaussinanos com matriz de covariância $\mathbf{\Sigma}$ conhecida então

$$
\sqrt{T} \mathbf{H}(\hat{\beta}-\beta) \stackrel{a}{\sim} Z
$$

onde $Z$ é uma vetor aleatório com ditribuição normal $k$-dimensional com média nula e matriz de covariância $\boldsymbol{\Gamma}$,

$$
\boldsymbol{\Gamma}=\lim _{N \rightarrow \infty} N \mathbf{H} E\left[\mathbf{D}^{\prime} \mathbf{D}\right]^{-1} \mathbf{H}^{\prime}
$$

$\mathbf{H}$ é uma matriz com $k$ linhas e mesmo número de colunas de $\mathbf{D}$, e $N=T-p$ é o número de linhas da matriz $\mathbf{X}_{t}$.

Observação: A matriz $\mathbf{H}$ é utilizada apenas para formalizar a convergência em distribuição. Como o número de parâmetros tende ao infinito quando o tamanho amostral $(T)$ aumenta, a matriz $H$ é útil para obtermos apenas uma subpartição finita destes parâmetros, cujas dimensões não dependem de $T$.

Prova:

Temos

$$
\begin{aligned}
\hat{\beta} & =\left(\mathbf{D}^{\prime} \mathbf{D}\right)^{-1} \mathbf{D}^{\prime} V \\
& =\left(\mathbf{D}^{\prime} \mathbf{D}\right)^{-1} \mathbf{D}^{\prime}(\mathbf{D} \beta+S+e) \\
& =\beta+\left(\mathbf{D}^{\prime} \mathbf{D}\right)^{-1} \mathbf{D}^{\prime} e+\left(\mathbf{D}^{\prime} \mathbf{D}\right)^{-1} \mathbf{D}^{\prime} S \\
& =\beta+T_{1}+T_{2} .
\end{aligned}
$$

Para $T_{2}$, o termo de erro devido ao truncamento na escala $J=J(T)$, analogamente a 
Donoho et al.(1995, teorema 8) e Dahlhaus et al. (1999, lema 8.3(i)) temos

$$
\begin{aligned}
\sup \left(\sum_{j \geq J} \sum_{k}\left\|\beta_{j, k}^{(i)}\right\|^{2}\right) & =O\left(T^{-\frac{2 m_{i}}{2 m_{i}+1}}\right) \\
T_{2} & =\left(\mathbf{D}^{\prime} \mathbf{D}\right)^{-1} \mathbf{D}^{\prime} S \\
\left\|T_{2}\right\|_{2} & \leq\left\|\left(\mathbf{D}^{\prime} \mathbf{D}\right)^{-1}\right\|_{2}\left\|\mathbf{D}^{\prime} S\right\|_{2} \\
& =O_{p}\left(\left(2^{-J \min \{\tilde{s}\}_{i}}+T^{-1 / 2} 2^{-J \min \left\{m_{i}-1 / 2-1 /\left(2 p_{i}\right)\right\}}\right) \sqrt{\log (T)}\right) \\
& =O_{p}\left(T^{-1 / 2-\tau(J)}\right),
\end{aligned}
$$

com $\tau(J)>0$. Note que $\sqrt{T}\left\|T_{2}\right\|_{2}=O_{p}\left(T^{-\tau(J)}\right)=o_{p}(1)$. Além disso, para derivarmos a distribuição assintótica de $\beta$, temos do Lema (4.3.6), que

$$
N \mathbf{H}\left(\mathbf{D}^{\prime} \mathbf{D}\right)^{-1} \mathbf{H}^{\prime} \stackrel{P}{\rightarrow} \boldsymbol{\Gamma}
$$

onde

$$
\boldsymbol{\Gamma}=\lim _{N \rightarrow \infty} N \mathbf{H} E\left[\left(\mathbf{D}^{\prime} \mathbf{D}^{-1}\right)\right] \mathbf{H}^{\prime}
$$

Assim, do Lema (4.3.7) e do teorema de Slutsky (Teorema 4.3.3), temos que

$$
\sqrt{T} \mathbf{H}(\hat{\beta}-\beta)=\sqrt{T} T_{1}+o_{p}(1) \sqrt{T} \mathbf{H}(\hat{\beta}-\beta)=Z+o_{p}(1)
$$

onde $Z$ é uma vetor aleatório com ditribuição normal $k$-dimensional com média nula e matriz de covariância $\Gamma$. Dessa forma, aplicando novamente o teorema de Slutsky (Teorema 4.3.3), o resultado segue.

Note que os estimadores de todos os coeficientes estão em $\hat{\beta}$. Assim, considere um subvetor $\hat{\xi}$ contendo todos os estimadores dos coeficientes de uma expansão de ondaletas de uma função $f(t)$, isto é, se $f(t)$ for uma função autoregressiva $\left(f(t)=a_{i, j}^{(l)}(t), l=\right.$ $1,2, \ldots, p, i, j=1,2, \ldots, s)$ ou se for função intercepto $\left(f(t)=u_{j}(t), j=1,2, \ldots, s\right)$. Dessa forma, um estimador natural para esta função $f(t)$ é

$$
\hat{f}(t)=\hat{\xi}^{\prime} \psi(t)=\sum_{j=0}^{J} \sum_{k} \hat{\beta}_{j, k} \psi_{j, k}(t),
$$

onde $\psi(t)$ é um vetor contendo as funções de ondaletas respectivas aos coeficientes da expansão em $\hat{\xi}$ avaliadas no instante $t$. Considere $\boldsymbol{\Sigma}_{\hat{\xi}}$ uma subpartição da matriz de covariância de $\hat{\beta}$, respectiva aos coeficientes em $\hat{\xi}$. 


\section{Proposição 4.1.2.}

$$
(\hat{f}(t)-f) \stackrel{a}{\sim} N\left(0, \psi \boldsymbol{\Sigma}_{\hat{\xi}} \psi^{\prime}\right)
$$

Prova: Resultado imediato da Proposição 4.1.1.

Assim como no caso estacionário, a causalidade de Granger variando no tempo é baseada no fato de que o efeito não pode preceder a causa, logo, as informações a respeito de causalidade estão nas relações entre o passado e futuro das séries. Considere duas séries temporais $x_{t}$ e $y_{t}$, realizações de dois processos localmente estacionários. Dizemos que $x_{t}$ Granger causa $y_{t}$ no instante $s$ se os valores passados de $x_{t}$ ajudam na previsão de $y_{s}$, ou seja, $x_{t}$ possui informações a respeito de $y_{s}$. Formalmente, se denotarmos o conjunto de todas as informações até o instante $s$ por $\Omega_{s}$ e o erro de predição de $y_{s}$ por $\Sigma_{y}\left(s \mid \Omega_{s}\right)$, dizemos que $x_{t}$ causa $y_{s}$ se

$$
\Sigma_{y}\left(s \mid \Omega_{s}\right)<\Sigma_{y}\left(s \mid \Omega_{s} /\left\{x_{t}, t<s\right\}\right) .
$$

Proposição 4.1.3. Se $z_{t}=\left[x_{t}, y_{t}\right]^{\prime}$ é um processo DVAR $(p)$ bivariado com matriz de covariância $\boldsymbol{\Sigma}(t)$ não singular, e $\hat{y}(s)$ o valor previsto da série y no instante $s$, temos $\hat{y}\left(s \mid\left\{z_{t}, t<s\right\}\right)=\hat{y}\left(s \mid\left\{z_{t}, t<s\right\}-\left\{x_{j t}, t<s\right\}\right) \Leftrightarrow A_{x \rightarrow y}^{l}(s)=0$, para $l=1, \ldots, p$.

Prova: O valor previsto de $z_{t}$ no instante $s$ é dado por

$$
E\left[z_{s} \mid\left\{z_{t}, t<s\right\}\right]=u(s)+\mathbf{A}_{1}(s) x_{s-1}+\mathbf{A}_{2}(s) x_{s-2}+\mathbf{A}_{p}(s) x_{s-p},
$$

$\log \mathrm{E} E\left[y_{s} \mid\left\{z_{t}, t<s\right\}\right]=E\left[y_{s} \mid\left\{y_{t}, t<s\right\}\right]$, para todo $s$, se e somente se, todos os coeficientes de causalidade de $x$ para $y$, denotados por $A_{x \rightarrow y}^{l}(t)$, forem iguais a zero no instante $s$. Em outras palavras, a série $y$ no instante $s$ não depende do passado de $x$.

Dessa forma, o modelo DVAR possibilita uma identificação natural da causalidade de Granger variando no tempo, através do teste da significância dos coeficientes do modelo.

\subsubsection{Teste de Hipóteses}

Considere um estimador para a variância dos erros no modelo transformado, dado por

$$
\begin{aligned}
\hat{\sigma}^{2} & =\left(\frac{1}{s\left[(T-p)-p 2^{J}\right]}\right)\left[\mathbf{D}^{\prime} \mathbf{D}-\hat{\beta}^{\prime} \mathbf{D}^{\prime} Y\right] \\
& =\left(\frac{1}{s\left[(T-p)-p 2^{J}\right]}\right) X_{t}^{\prime}\left[\boldsymbol{\Sigma}^{-1}-\boldsymbol{\Sigma}^{-1} \mathbf{M}\left(\mathbf{M}^{\prime} \boldsymbol{\Sigma}^{-1} \mathbf{M}\right)^{-1} \mathbf{M}^{\prime} \boldsymbol{\Sigma}^{-1}\right] X_{t} .
\end{aligned}
$$


A matriz de covariância estimada de $\hat{\beta}$ é dada por:

$$
\operatorname{va} r(\hat{\beta})=\hat{\sigma}^{2}\left(\mathbf{M}^{\prime} \mathbf{\Sigma}^{-1} \mathbf{M}\right)^{-1}
$$

A estatística de teste de Wald para contrastes é

$$
W=\frac{(\mathbf{C} \hat{\beta}-m)^{\prime}\left[\mathbf{C}\left(\mathbf{D}^{\prime} \mathbf{D}\right)^{-1} \mathbf{C}^{\prime}\right]^{-1}(\mathbf{C} \hat{\beta}-m)}{\hat{\sigma}},
$$

onde $m$ é um vetor e $\mathbf{C}$ é uma matriz de contrastes do teste correspondente. Queremos realizar o seguinte teste de hipóteses:

$$
\begin{aligned}
& H_{0}: \mathbf{C} \beta=m, \\
& H_{A}: \mathbf{C} \beta \neq m .
\end{aligned}
$$

Sob a hipótese nula, a estatística $W$ possui distribuição assintótica qui-quadrado com posto $(C)$ graus de liberdade.

De fato, sob a hipótese nula, segue da Proposição 4.1.1 que se C é não singular e aplicando o teorema de Cochran (Sen \& Singer, 1993), a estatística $W$ tem distribuição qui-quadrado com posto $(C)$ graus de liberdade.

\subsubsection{Estimação da Matriz de Covariâncias}

Na prática, a matriz de covariância dos erros aleatórios $\boldsymbol{\Sigma}$ não é conhecida. Assim sendo, considere $\epsilon_{l t}$ o termo de erros aleatórios de uma série temporal $x_{l t}$ univariada com esperança nula e variância $\sigma_{l}^{2}(t)$ variante no tempo. Sabemos que

$$
\begin{aligned}
E\left(\epsilon_{l t}^{2}\right) & =\operatorname{Var}\left(\epsilon_{l t}^{2}\right)+E\left(\epsilon_{l t}\right)^{2} \\
& =\operatorname{Var}\left(\epsilon_{l t}^{2}\right)=\sigma_{l}^{2}(t) .
\end{aligned}
$$

Dessa forma, um estimador natural de $\sigma_{l}^{2}(t)$ é o quadrado dos resíduos,

$$
\begin{aligned}
E\left(r_{l t}^{2}\right) & =E\left[\left(S_{l t}+\epsilon_{l t}\right)^{2}\right]+o_{p}(1) \\
& =E\left(\epsilon_{l t}^{2}\right)+2 E\left(S_{l t} \epsilon_{l t}\right)+E\left(S_{l t}^{2}\right)+o_{p}(1),
\end{aligned}
$$

onde $S_{l t}=o(1)$ é o termo de erro devido ao truncamento na escala $J(T)$. Utilizando os resultados de Dahlhaus (1999), $S_{l t} \rightarrow 0$ para $J(T)$ suficientemente grande, de forma que

$$
\lim _{T \rightarrow \infty} E\left(r_{l t}^{2}\right)=\sigma_{l}^{2}(t)
$$


Analogamente, um estimador para a função de covariância $\sigma_{l m}^{2}(t)$ variante no tempo de duas séries temporais $x_{l t}$ e $x_{m t}$ é dado por $r_{l t} r_{m t}$. Utilizando a expansão em ondaletas, os estimadores para as funções de variância e covariância são obtidos através de um processo de suavização por ondaletas (truncamento em uma escala $J(T)$ ), da forma

$$
\begin{aligned}
\hat{\sigma}_{l}^{2}(t) & =\sum_{j} \sum_{k} v_{j, k} \psi_{j, k}(x), \\
\hat{\sigma}_{l m}(t) & =\sum_{j} \sum_{k} c_{j, k} \psi_{j, k}(x),
\end{aligned}
$$

onde os coeficientes $v_{j, k}$ e $c_{j, k}$ podem ser obtidos através de procedimentos de mínimos quadrados ordinários, resultando em um estimador consistente para $\Sigma$.

Dessa forma, dados os resíduos do modelo, é possível estimar a variância variante no tempo de cada uma das séries temporais univariadas e também as funções de covariância variantes no tempo, ou seja, é possível estimar $\boldsymbol{\Sigma}(t)$ consistentemente. Assim, propomos um algorítmo de mínimos quadrados generalizados iterativo, definido por:

- Passo 1) Estime o modelo DVAR utilizando um procedimento de mínimos quadrados ordinários (MQO), ou seja, assuma que $\boldsymbol{\Sigma}=\mathbf{I}$;

- Passo 2) Estime as variâncias univariadas e funções de covariâncias através de MQO ou transformada discreta de ondaletas limiarizada;

- Passo 3) Re-estime o modelo DVAR por mínimos quadrados generalizados (MQG) considerando $\hat{\boldsymbol{\Sigma}}$ estimada no passo anterior;

- Passo 4) Volte ao passo 2 e itere até a convergência dos parâmetros. Note que o algorítmo é uma extensão do procedimento de Cochrane-Orcutt, de forma que a variância dos estimadores será menor que utilizando um procedimento de mínimos quadrados ordinários.

Proposição 4.1.4. Seja $\hat{\Sigma}$ um estimador consistente para a matriz de covariância dos erros $\boldsymbol{\Sigma} e$

$$
\hat{\beta}_{\hat{\Sigma}}=\left(\mathbf{M}^{\prime} \hat{\Sigma}^{-1} \mathbf{M}\right)^{-1} \mathbf{M}^{\prime} \hat{\Sigma}^{-1} Y
$$

um estimador para $\beta$, no caso em que a matriz $\Sigma$ não é conhecida. Então a distribuição assintótica de $\hat{\beta}_{\hat{\Sigma}}$ é a mesma de $\hat{\beta}$.

Prova: Como $\hat{\Sigma}$ é um estimador consistente, dadas as propriedades do estimador de 
mínimos quadrados, então $\hat{\Sigma}-\Sigma \stackrel{P}{\rightarrow} 0$. Logo,temos

$$
\begin{aligned}
T\left(\mathbf{M}^{\prime} \hat{\boldsymbol{\Sigma}}^{-1} \mathbf{M}\right)^{-1} & =T\left(\mathbf{M}^{\prime}[\hat{\boldsymbol{\Sigma}}-\boldsymbol{\Sigma}+\boldsymbol{\Sigma}]^{-1} \mathbf{M}\right)^{-1} \\
& =T\left(\mathbf{M}^{\prime} \boldsymbol{\Sigma}^{-1} \mathbf{M}\right)^{-1}+o_{p}(1)
\end{aligned}
$$

Logo, pelo teorema de Slustky, a distribuição assintótica de $T\left(\mathbf{M}^{\prime} \hat{\mathbf{\Sigma}} \mathbf{M}\right)^{-1}$ é a mesma de $T\left(\mathbf{M}^{\prime} \mathbf{\Sigma} \mathbf{M}\right)^{-1}$. Analogamente, verifica-se que a distribuição assintótica de $\left[\mathbf{M}^{\prime} \hat{\mathbf{\Sigma}}^{-1} Y\right] / T$ também é a mesma de $\left[\mathbf{M}^{\prime} \Sigma^{-1} Y\right] / T$. Dessa forma, o resultado segue.

Uma aplicação importante deste resultado é que todos os resultados anteriores em que a matriz de covariâncias dos erros era considerada conhecida, também são válidos se utilizarmos um estimador consistente para a mesma.

\subsection{Conectividade via DVAR}

Como foi visto anteriormente, modelos multivariados de séries temporais vêm sendo amplamente utilizados para a modelagem da conectividade e fluxo de informação cerebral. No entanto, a maioria os métodos assumem a estacionariedade das séries temporais, ou seja, os parâmetros do modelo não variam temporalmente. Dessa forma, introduzimos um novo método para a identificação e quantificação da conectividade cerebral utilizando os modelos DVAR baseado em ondaletas. Dessa forma, utilizando esta classe de modelo é possível visualizar até mesmo as alterações na conectividade durante a realização de uma determinada tarefa.

Assim, considerando experimentos de RMf, onde cada indivíduo realiza várias tarefas distintas em uma mesma sessão, o modelo proposto permite a identificação da intensidade e direção do fluxo de informações ao longo do experimento. Além disso, através da modelagem da estrutura de conectividade variante no tempo, pode-se estimar o tempo de atraso para a alteração da conectividade e também efeitos de habituação.

No entanto, sabemos que o sinal BOLD é apenas uma medida indireta da atividade neuronal, e como o modelo DVAR não é baseado em mecanismos neurofisiológicos, todas as inferências e análises obtidas através do modelo só dizem respeito à conectividade funcional entre as áreas envolvidas. Em outras palavras, as análises estão relacionadas somente à previsibilidade do sinal BOLD, e não em interações neuronais ou causalidade 
propriamente ditas. Contudo, informações da estrutura e vias anatômicas provenientes de estudos anteriores ou modelos neurofisiológicos podem ser introduzidas no modelo como restrições nos parâmetros. Maiores detalhes e exemplos de possíveis aplicações do modelo proposto à dados de RMf serão apresentados no capítulo 6.

\subsection{Apêndice}

\section{Definição 4.3.1. Seqüência de vetores diferenças martingais}

Seja $\mathscr{F}_{t}$ a sigma-álgebra contendo toda a informação disponível até o instante $t$. Um processo estocástico k-dimensional $Y_{t}$ é dito um vetor seqüência de diferenças martingais se $E\left(Y_{t} \mid \mathscr{F}_{t-1}\right)=0$.

Teorema 4.3.1. Teorema central do limite para seqüência de vetores diferenças martingais (White, 1984)

Seja $Y_{t}$ um vetor $k$-dimensional seqüência de diferenças martingais, com $\bar{Y}_{T}=(1 / T) \sum_{t=1}^{T} Y_{t}$, se

i) $E\left(Y_{t} Y_{t}^{\prime}\right)=\boldsymbol{\Gamma}_{t}$ matriz positiva definida, com $(1 / T) \sum_{t=1}^{T} \boldsymbol{\Gamma}_{t} \rightarrow \boldsymbol{\Gamma}$;

ii) $E\left(Y_{i t} Y_{j t} Y_{l t} Y_{m t}\right)<\infty \forall y, i, j, l, m\left(Y_{i t}\right.$ é o $i$-ésimo elemento do vetor $\left.Y_{t}\right)$;

iii) $(1 / T) \sum_{t=1}^{T} Y_{t} Y_{t}^{\prime} \stackrel{P}{\rightarrow} \boldsymbol{\Gamma}$;

Então $(1 / T) \bar{Y}_{T} \stackrel{\mathscr{D}}{\rightarrow} N(0, \Gamma)$.

\section{Definição 4.3.2. Processos uniformemente integráveis}

Um processo $Y$ é dito uniformemente integrável se $\forall \epsilon>0$ existe uma constante $M$ tal que $E\left(\left|Y \mathbf{I}_{|Y|>M \mid}\right|\right)<M$.

Definição 4.3.3. $L^{1}$-Mixingais (Andrew, 1988)

Considere uma seqüência de variáveis aleatórias $\left\{Y_{t}\right\}_{t=1}^{\infty} \operatorname{com} E\left(Y_{t}\right)=0$, para $t=1,2, \ldots$ Seja $\mathscr{F}_{t}$ a sigma-álgebra contendo toda a informação disponível até o instante $t$. Se existem seqüências não negativas de constantes $\left\{c_{t}\right\}_{t=1}^{\infty} e\left\{\xi_{t}\right\}_{t=1}^{\infty}$ tal que $\lim _{t \rightarrow \infty} \xi_{t}=0$ e

$$
E\left\|E\left(Y_{t} \mid \mathscr{F}_{t-m}\right)\right\|_{1} \leq c_{t} \xi_{m},
$$

$\forall t \geq 1$ e $\forall m \geq 0$, então $\left\{Y_{t}\right\}_{t=1}^{\infty}$ é dita um $L^{1}$-Mixingal com respeito à $\left\{\mathscr{F}_{t}\right\}_{t=1}^{\infty}$. 
Observação: Note que um L L-Mixingal é uma seqüência de variáveis aleatórias dependentes, mas assintóticamente independentes. Assim, processos estacionários e localmente estacionários podem ser considerados $\mathrm{L}^{1}$-Mixingais.

Teorema 4.3.2. Lei fraca dos grandes números para $L^{1}$-Mixingais (Andrew, 1988)

Seja $\left\{Y_{t}\right\}_{t=1}^{\infty}$ um $L^{1}$-Mixingal. Se $Y_{t}$ é uniformemente integrável e existe $c_{t}$ tal que

$$
\lim _{t \rightarrow \infty}(1 / T) \sum_{t=1}^{T} c_{t} \leq \infty
$$

então

$$
(1 / T) \sum_{t=1}^{T} Y_{t} \stackrel{P}{\rightarrow} 0 .
$$

Proposição 4.3.1. Condições suficientes para um $L^{1}$-Mixingal ser uniformemente integrável (Hamilton, p.191)

Suponha que existe $r>1$ e $M<\infty$ tal que $\left.E\left(\left|X_{t}\right|^{r}\right)<M\right)$. Se $Y_{t}=\sum_{j=-\infty}^{\infty} h_{t} X_{t-j}$ com $\sum_{j=-\infty}^{\infty}\left|h_{t}\right|<\infty$, então $Y_{t}$ é uniformemente integrável.

Observação: Note que este resultado é importante para a aplicação da lei fraca dos grandes números para $\mathrm{L}^{1}$-Mixingais, pois se $Y_{t}$ é uma série adequadamente filtrada de $X_{t}$, $Y_{t}$ é uniformemente integrável.

Teorema 4.3.3. Teorema de Slutsky (Sen e Singer, 1993)

Considere os processos estocásticos vetoriais $X_{t}$ e $Y_{t}$ satisfazendo:

$$
X_{t} \stackrel{P}{\rightarrow} C
$$

$e$

$$
Y_{t} \stackrel{\mathscr{D}}{\rightarrow} Z
$$

Então

i) $X_{t}+Y_{T} \stackrel{\mathscr{D}}{\rightarrow} C+Z$,

ii) $X_{t} Y_{T} \stackrel{\mathscr{D}}{\rightarrow} C Z$. 
Corolário 4.3.4. Técnica da transformação (Sen e Singer, 1993)

Considere a função $f: \mathbb{R}^{n} \rightarrow \mathbb{R}^{m}$, contínua no ponto $x_{0}$, então:

i) se $x \stackrel{\mathscr{D}}{\rightarrow} x_{0}$ então $f(x) \stackrel{\mathscr{D}}{\rightarrow} f\left(x_{0}\right)$,

ii) se $x \stackrel{P}{\rightarrow} x_{0}$ então $f(x) \stackrel{P}{\rightarrow} f\left(x_{0}\right)$,

iii) se $x \stackrel{\text { q.c. }}{\longrightarrow} x_{0}$ então $f(x) \stackrel{\text { q.c. }}{\longrightarrow} f\left(x_{0}\right)$.

Corolário 4.3.5. Seja $X_{n}$ uma seqüência de vetores aleatórios. Se

$$
X_{n}-E\left[X_{n}\right] \stackrel{P}{\rightarrow} 0
$$

$e$

$$
E\left[X_{n}\right]-\mu \stackrel{P}{\rightarrow} 0
$$

então $X_{n} \stackrel{P}{\rightarrow} \mu$.

Lema 4.3.6. Considerando que as condições (C1)-(C5) estão satisfeitas, temos que

$$
N \mathbf{H}\left(\mathbf{D}^{\prime} \mathbf{D}\right)^{-1} \mathbf{H}^{\prime} \stackrel{P}{\rightarrow} \boldsymbol{\Gamma},
$$

onde

$$
\boldsymbol{\Gamma}=\lim _{N \rightarrow \infty} N \mathbf{H} E\left[\mathbf{D}^{\prime} \mathbf{D}\right]^{-1} \mathbf{H}^{\prime}
$$

e $N$ é o número de linhas da matriz $\mathbf{X}_{t}$.

Observação: Note que cada elemento de $\lim _{N \rightarrow \infty} E\left[\mathbf{D}^{\prime} \mathbf{D}\right] / N$ é da forma $\lim _{N \rightarrow \infty} \sum_{t=1}^{N} f(t / N) / N=$ $\int_{0}^{1} f(t) d t$, pois é um caso particular da integral de Riemann $(\Delta=1 / N)$. Prova:

Primeiramente, note que a matriz $\mathbf{D}$ é bloco diagonal. Seja $d_{i j}$ o elemento na $i$-ésima linha, na $j$-ésima coluna de um bloco não nulo da matriz modelo $\mathbf{D}$. Note que devido a transformação dos mínimos quadrados generalizados (transformação ortonormalizadora), $d_{i j}$ é da forma

$$
d_{t j}=f_{t j} X_{t j}
$$

onde $f_{t j}$ é uma função resultante do produto entre uma função de ondaletas e a função da transformação ortonormalizadora, e $X_{t j}$ é um processo localmente estacionário. 
Defina $(d d)_{l m}$ o elemento da $l$-ésima linha, na $m$-ésima coluna de qualque subpartição da matriz $\mathbf{D}^{\prime} \mathbf{D}$. Assim, $(d d)_{l m}$ é da forma

$$
\begin{aligned}
(d d)_{l m} & =\sum_{t=1}^{N} d_{t l} d_{t m}=\sum_{t=1}^{N} f_{t l} X_{t l} f_{t m} X_{t m} \\
& =\sum_{t=1}^{N} f_{t l} f_{t m} \sum_{j=0}^{\infty} \sum_{v=0}^{\infty} \theta_{l j}(t) \theta_{m v}(t) \epsilon_{l,(t-j)} \epsilon_{m,(t-v)} \\
& +\sum_{t=1}^{N} f_{t l} f_{t m} \mu_{l}(t) \sum_{v=0}^{\infty} \theta_{m v}(t) \epsilon_{m,(t-v)}+\sum_{t=1}^{N} f_{t l} f_{t m} \mu_{m}(t) \sum_{j=0}^{\infty} \theta_{l j}(t) \epsilon_{l,(t-j)} \\
& +\sum_{t=1}^{N} f_{t l} f_{t m} \mu_{m}(t) \mu_{l}(t) .
\end{aligned}
$$

Considere agora

$$
\begin{aligned}
z_{l m t}^{(1)} & =\sum_{t=1}^{N} f_{t l} f_{t m} \sum_{j=h}^{\infty} \sum_{v=0}^{\infty} \theta_{l j}(t) \theta_{m v}(t)\left[\epsilon_{l,(t-j)} \epsilon_{m,(t-v)}-E\left[\epsilon_{l,(t-j)} \epsilon_{m,(t-v)}\right]\right], \\
z_{l m t}^{(2)} & =\sum_{t=1}^{N} f_{t l} f_{t m} \mu_{l}(t) \sum_{v=0}^{\infty} \theta_{m v}(t) \epsilon_{m,(t-v)}, \\
z_{l m t}^{(3)} & =\sum_{t=1}^{N} f_{t l} f_{t m} \mu_{m}(t) \sum_{j=0}^{\infty} \theta_{l j}(t) \epsilon_{l,(t-j)},
\end{aligned}
$$

Assim, temos que

$$
\begin{aligned}
E\left[z_{l m t}^{(1)} \mid \mathscr{F}_{t-h}\right] & =\sum_{t=1}^{N} f_{t l} f_{t m} \sum_{j=h}^{\infty} \sum_{v=h}^{\infty}\left(\theta_{l j}(t) \theta_{m v}(t) \epsilon_{l,(t-j)} \epsilon_{m,(t-v)}-E\left[\epsilon_{l,(t-j)} \epsilon_{m,(t-v)}\right]\right) \\
E\left\|E\left[z_{l m t}^{(1)} \mid \mathscr{F}_{t-h}\right]\right\| & \leq \sum_{t=1}^{N}\left|f_{t l} f_{t m}\right| \sum_{j=h}^{\infty} \sum_{v=h}^{\infty}\left|\theta_{l j}(t)\right|\left|\theta_{m v}(t)\right| E\left\|\left[\epsilon_{l,(t-j)} \epsilon_{m,(t-v)}-E\left[\epsilon_{l,(t-j)} \epsilon_{m,(t-v)}\right]\right]\right\| \\
& \leq \sum_{j=h}^{\infty} \sum_{v=h}^{\infty}\left|\theta_{l j}(t)\right|\left|\theta_{m v}(t)\right| K=\xi_{l m h} .
\end{aligned}
$$

Como a seqüência $\left\{\theta_{j}(t)\right\}_{j=0}^{\infty}$ é absolutamente somável, temos

$$
\lim _{h \uparrow \infty} \xi_{l m h}=0,
$$

e tomando $c_{t}=1$, concluimos que $z_{l m t}^{(1)}$ um $\mathrm{L}^{1}$-Mixingal com respeito a $\mathscr{F}_{t}$. Analogamente, temos que $z_{l m t}^{(2)}$ e $z_{l m t}^{(3)}$ também são $\mathrm{L}^{1}$-Mixingais. Além disso, como o vetor $\epsilon_{t}$ é gaussiano, 
segue que

$$
\begin{aligned}
E\left[\| \epsilon_{l,(t-j)} \epsilon_{m,(t-v)}-\left.E\left[\epsilon_{l,(t-j)} \epsilon_{m,(t-v)}\right]\right|^{4}\right] & <\infty \\
E\left[\left|\epsilon_{l},(t-j)\right|^{4}\right] & <\infty \\
E\left[\left|\epsilon_{m},(t-v)\right|^{4}\right] & <\infty
\end{aligned}
$$

Assim, utilizando o teorema da lei fraca dos grandes números para $\mathrm{L}^{1}$-Mixingais, a Proposição (4.2.1), o Corolário (4.2.5) e a técnica da transformação (Corolário (4.2.4)), temos que

$$
N \mathbf{H}\left(\mathbf{D}^{\prime} \mathbf{D}\right)^{-1} \mathbf{H}^{\prime} \stackrel{P}{\rightarrow} \boldsymbol{\Gamma}
$$

Lema 4.3.7. Considerando que as condições (C1)-(C5) estão satisfeitas, temos que

$$
(1 / \sqrt{N}) \mathbf{G}\left(\mathbf{D}^{\prime} e\right) \stackrel{D}{\rightarrow} Z
$$

onde $Z$ é uma variável aleatória com distribuição normal multivariada com média nula e matriz de covariâncias $\boldsymbol{\Gamma}^{*-1}=\lim _{N \rightarrow \infty} \frac{\mathbf{G} E\left[\mathbf{D}^{\prime} \mathbf{D}\right] \mathbf{G}^{\prime}}{N}, N=T-p$, e $\mathbf{G}$ é uma matriz com $k$ linhas e mesmo número de colunas de $(\mathbf{D})$.

Prova:

Seja $(d e)_{j}$ o elemento na $j$-ésima linha de $\left(\mathbf{D}^{\prime} e\right)$, que é da forma

$$
(d e)_{j}=\sum_{t=1}^{N}(d e)_{j t}=\sum_{t=1}^{N} h_{t j} X_{t j} e_{t},
$$

onde $(d e)_{j t}=h_{t j} X_{t j} e_{t},\left\{e_{t}\right\}_{t=1}^{N}$ são processos gaussianos independentes, $X_{t j}$ é um processo localmente estacionário e $h_{t j}$ é uma função resultante do produto entre uma função de ondaletas e uma função decorrente do processo de ortonormalização.

Note que $\mathbf{D}^{\prime} e$ é um vetor composto por elementos $(d e)_{j}$, e portanto pode ser escrito como $\mathbf{D}^{\prime} e=\sum_{t=1}^{N} b_{t}$, onde $b_{t}$ é um vetor composto por elementos $(d e)_{j t}$.

Temos também que

$$
E\left[b_{t} \mid \mathscr{F}_{t-1}\right]=0
$$


e portanto $\left\{b_{t}\right\}_{t=1}^{\infty}$ é uma seqüência de vetores diferença martingal. Além disso,

$$
\begin{gathered}
E\left[\frac{\sum_{t=1}^{N} b_{t} b_{t}^{\prime}}{N}\right]=E\left[\frac{\mathbf{D}^{\prime} e e^{\prime} \mathbf{D}}{N}\right]=E\left[E\left[\frac{\mathbf{D}^{\prime} e e^{\prime} \mathbf{D}}{N} \mid \mathbf{D}\right]\right]=E\left[\frac{\mathbf{D}^{\prime} \mathbf{D}}{N}\right], \\
\mathbf{G} E\left[\frac{\sum_{t=1}^{N} b_{t} b_{t}^{\prime}}{N}\right] \mathbf{G}^{\prime}=\mathbf{G} E\left[\frac{\mathbf{D}^{\prime} \mathbf{D}}{N}\right] \mathbf{G}^{\prime} \rightarrow \mathbf{\Gamma}^{*-1},
\end{gathered}
$$

onde

$$
\mathbf{\Gamma}^{*-1}=\lim _{N \rightarrow \infty} \frac{\mathbf{G} E\left[\mathbf{D}^{\prime} \mathbf{D}\right] \mathbf{G}^{\prime}}{N} .
$$

Analogamente à prova do Lema (4.2.6), temos que

$$
\mathbf{G} \frac{\sum_{t=1}^{N} b_{t} b_{t}^{\prime}}{N} \mathbf{G}^{\prime} \stackrel{P}{\rightarrow} \Gamma^{*-1} .
$$

Assim, utilizando teorema limite central para seqüencia de vetores diferenças martingais, o resultado segue. 
CApítulo 5

\section{Simulações}

Neste capítulo, serão apresentados alguns resultados empíricos a respeito do viés e variância do estimador de mínimos quadrados dos parâmetros do modelo DVAR. Também realizamos simulações visando avaliar a distribuição dos estimadores do modelo e o poder do teste de Wald para coeficientes variando no tempo. Todos os resultados apresentados nesta seção são baseados em técnicas de simulação e computação intensiva. As conclusões a respeito dos conjuntos de simulações são apresentadas conjuntamente no final deste capítulo.

É importante ressaltar que todos os conjuntos de simulações deste capítulo foram realizadas visando avaliar a aplicabilidade das metodologias propostas a dados de ressonância magnética funcional. Para este tipo de dados, não temos séries temporais longas (entre 50 e 200 volumes). Dessa forma, como o objetivo deste trabalho é o desenvolvimento de uma nova ferramenta de análise, consideramos séries pequenas. Além disso, também enfrentamos limites computacionais para a viabilização das análises. Utilizando um computador Intel Pentium 4, são necessárias de 3 a 4 horas para a realização de cada conjunto de 500 simulações com 256 pontos no tempo.

\subsection{Simulação 1}

Neste primeiro conjunto de simulações, visamos avaliar a eficiência dos estimadores propostos considerando um modelo DVAR, onde a tendência determinística (intercepto) de cada série e os coeficientes de causalidade são funções suaves no tempo. Assim sendo, 
considere um processo DVAR bivariado dado por

$$
x_{t}=u(t)+\mathbf{A}(t) x_{t-1}+\epsilon_{t},
$$

onde

$$
\mathbf{u}(t)=\left(\begin{array}{c}
\operatorname{sen}\left(\frac{2 \pi t}{128}+\pi\right) \\
-0,6
\end{array}\right), \mathbf{A}(t)=\left(\begin{array}{cc}
0,5-\frac{\operatorname{sen}\left(\frac{2 \pi t}{128}\right)}{4} & -0,5+\frac{\cos \left(\frac{2 \pi t}{128}\right)}{4} \\
0,5-\frac{\operatorname{sen}\left(\frac{\pi t}{128}\right)}{4} & -0,3
\end{array}\right)
$$

e

$$
\boldsymbol{\Sigma}(t)=\left(\begin{array}{cc}
1,4 & 1,4\left(1+\frac{\cos \left(\frac{2 \pi t}{128}\right)}{4}\right) \\
1,4\left(1+\frac{\cos \left(\frac{2 \pi t}{128}\right)}{4}\right) & 2\left(1+\frac{\cos \left(\frac{2 \pi t}{128}\right)}{4}\right)^{2}
\end{array}\right),
$$

onde $u(t)$ é o vetor de interceptos, $\mathbf{A}(t)$ é a matriz de coeficientes autoregressivos e $\boldsymbol{\Sigma}(t)$ é a matriz de covariância dos erros aleatórios. Este processo foi simulado 500 vezes, onde para cada uma das simulações realizou-se a estimação via mínimo quadrados proposta, considerando como base de funções as ondaletas de Haar e também as Daublets 8 (o truncamento foi realizado na escala 2, resultando em 4 funções como base). As séries simuladas possuem extensão $T=128$ e considerou-se vetor de erros com distribuição normal multivariada. Além disso para verificar a distribuição dos estimadores, construimos histogramas e qq-plots para comparações com a distribuição gaussiana. Os resultados são apresentados nas Figura 5.1 à 5.12 .

\subsection{Simulação 2}

Além da avaliação do viés e variância dos estimadores propostos, desejamos também avaliar a performance do teste estatístico para coeficientes de causalidade variando no tempo. Considerando as wavelets D8 (double extreme phase) com condições de borda periódica e Haar, temos que a ondaleta pai $\psi_{-1,0}(t)$ é uma função constante. Dessa forma, se a função autoregressivade causalidade é dada por

$$
a(t)=\sum_{j} \sum_{k} a_{j, k} \psi_{j, k}(t)
$$

o teste estatístico para função de causalidade variando no tempo

$H_{0}$ : O coeficiente de causalidade é invariante no tempo; 

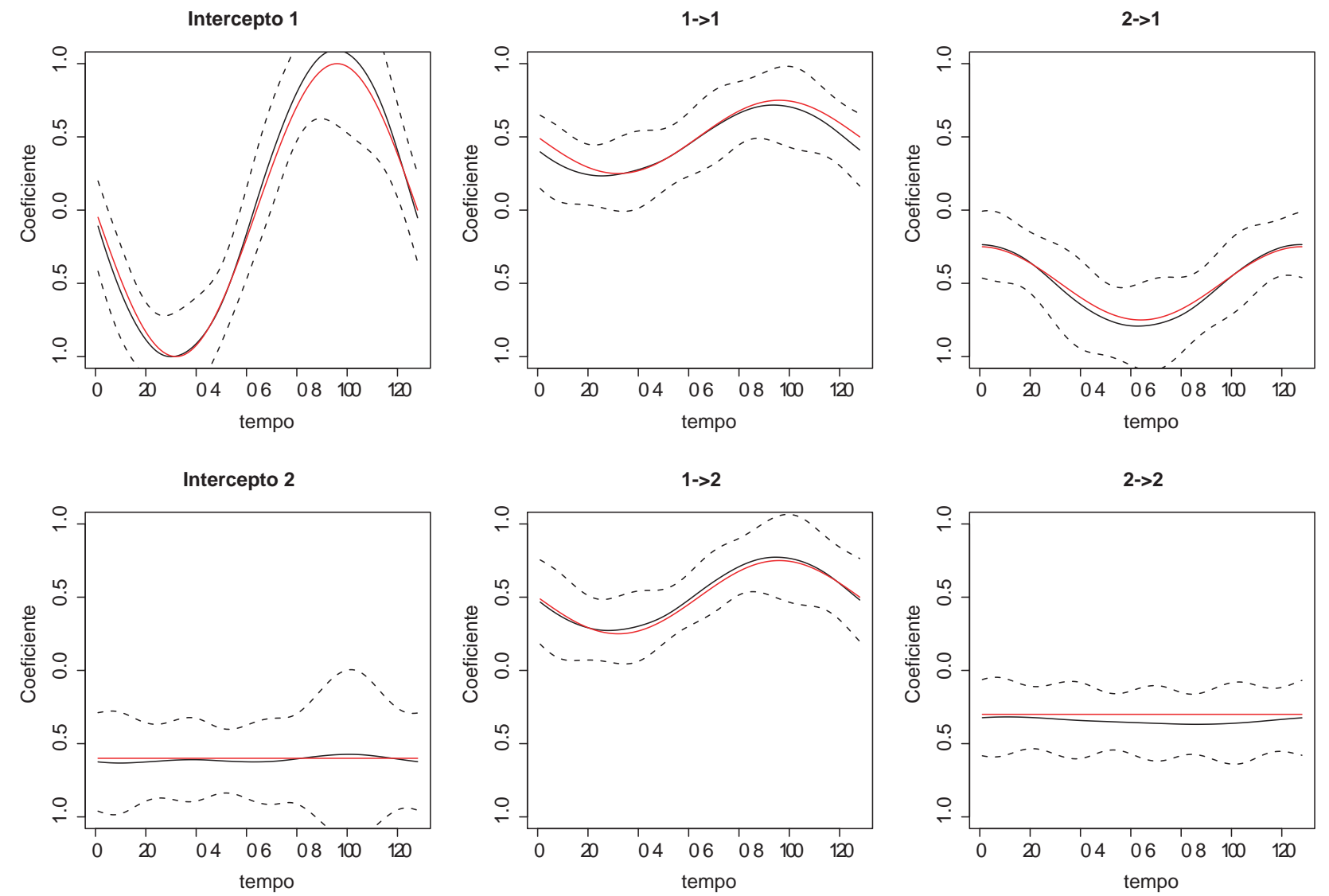

Figura 5.1: Simulação 1: Coeficientes do modelo DVAR utilizando as ondaletas Daublets 8. A linha vermelha e preta indicam os valores das curvas verdadeiras e esperanças estimadas, respectivamente. As linhas tracejadas indicam um intervalo de 1 erro padrão. 

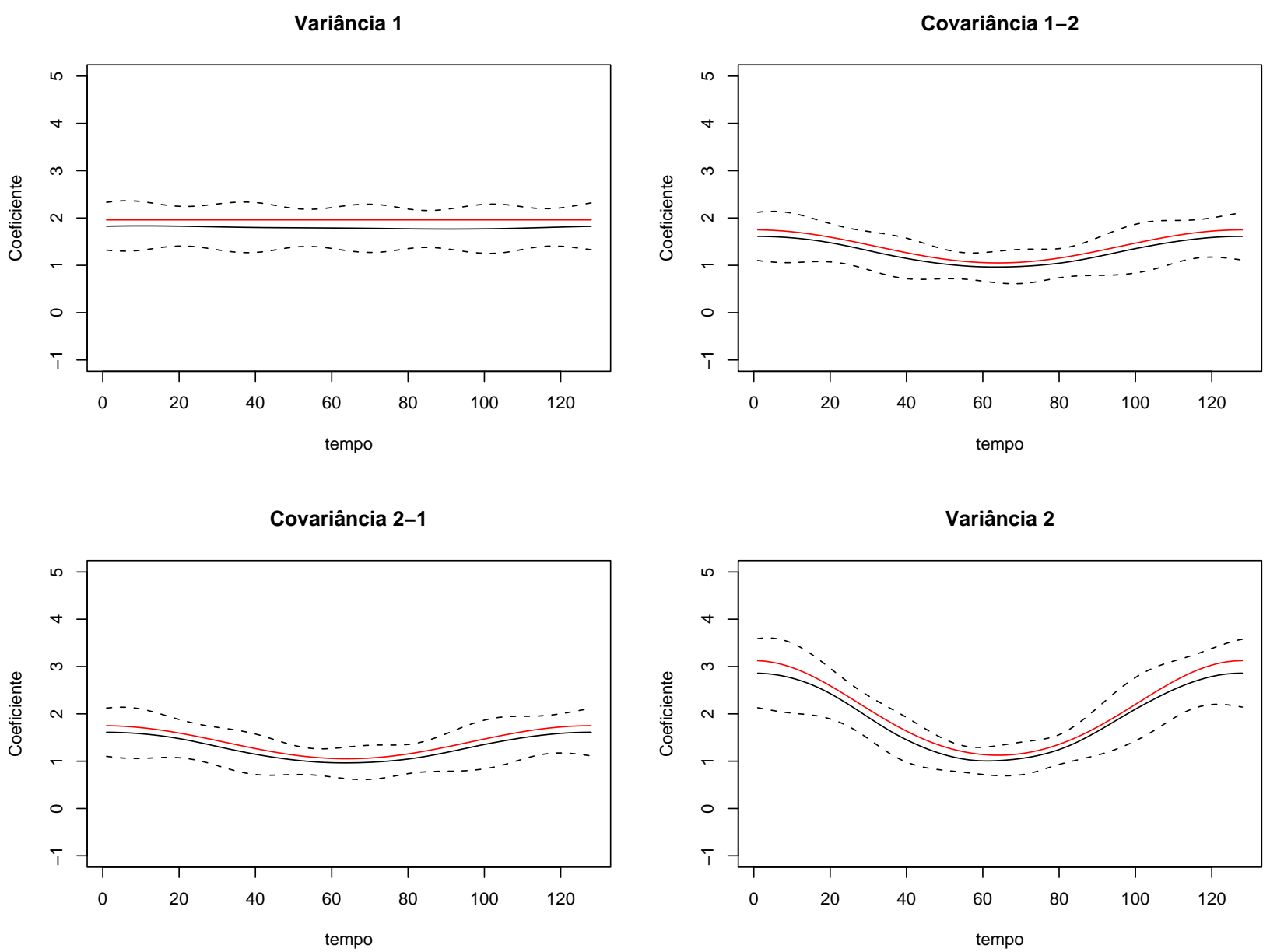

Figura 5.2: Simulação 1: Matriz de Covariância dos Erros do modelo DVAR utilizando as ondaletas Daublets 8. A linha vermelha e preta indicam os valores das curvas verdadeiras e esperanças estimadas, respectivamente. As linhas tracejadas indicam um intervalo de 1 erro padrão. 


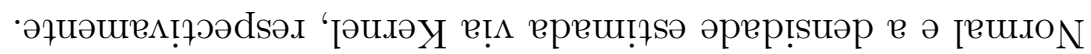

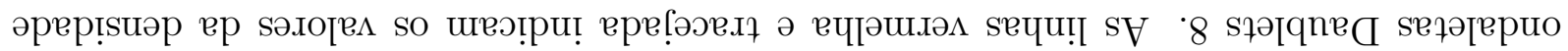

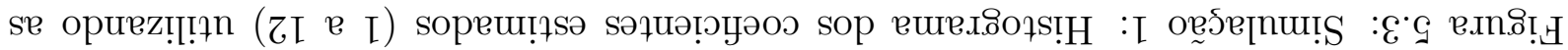
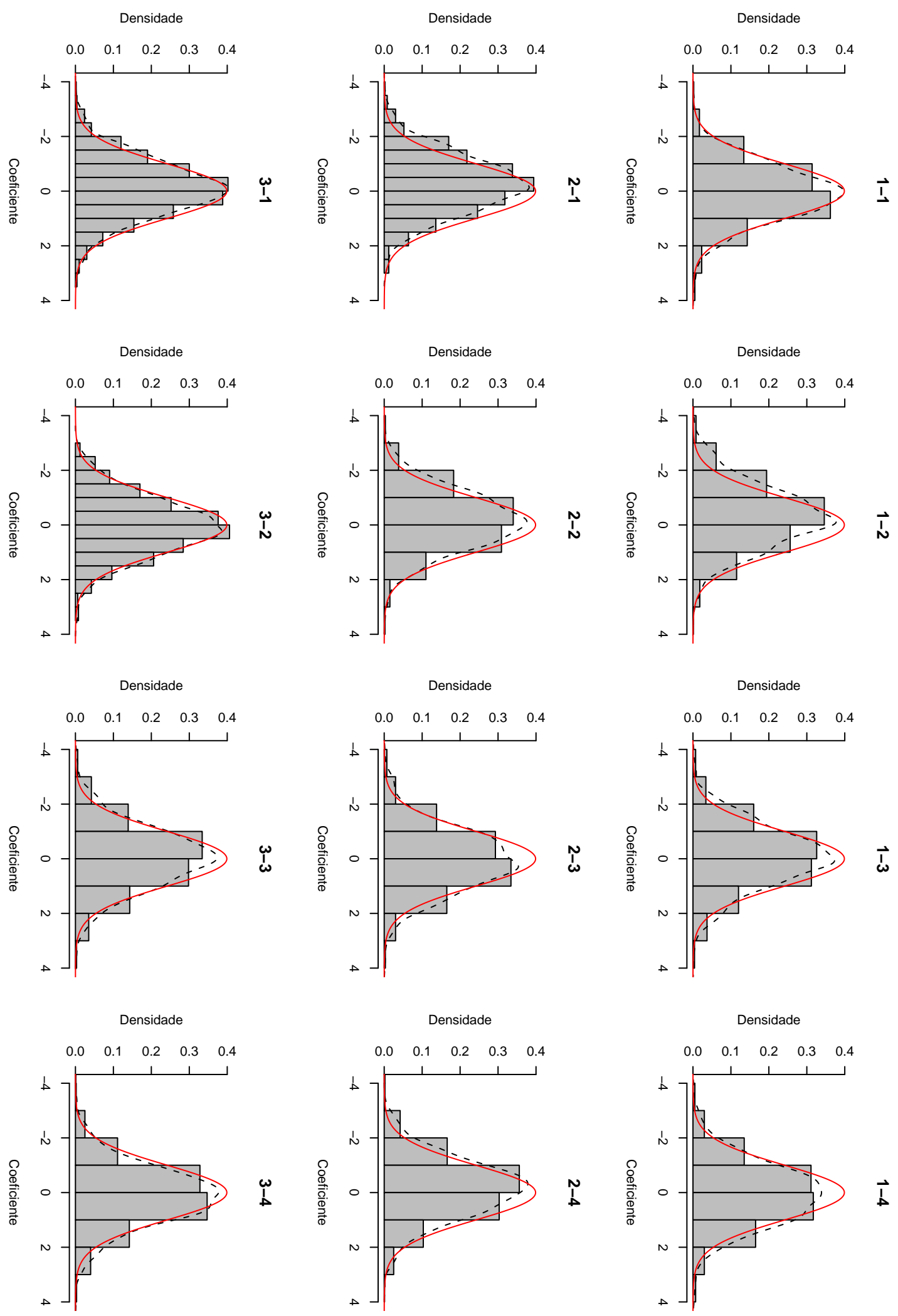


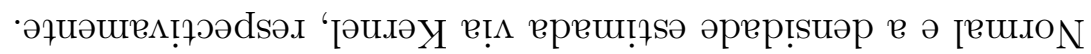

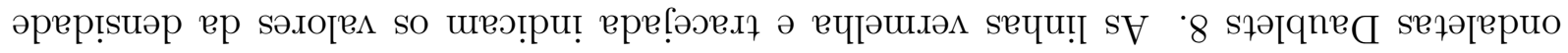

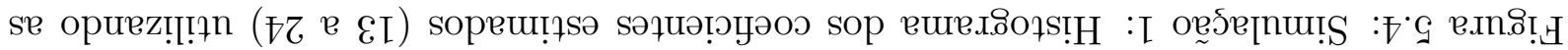
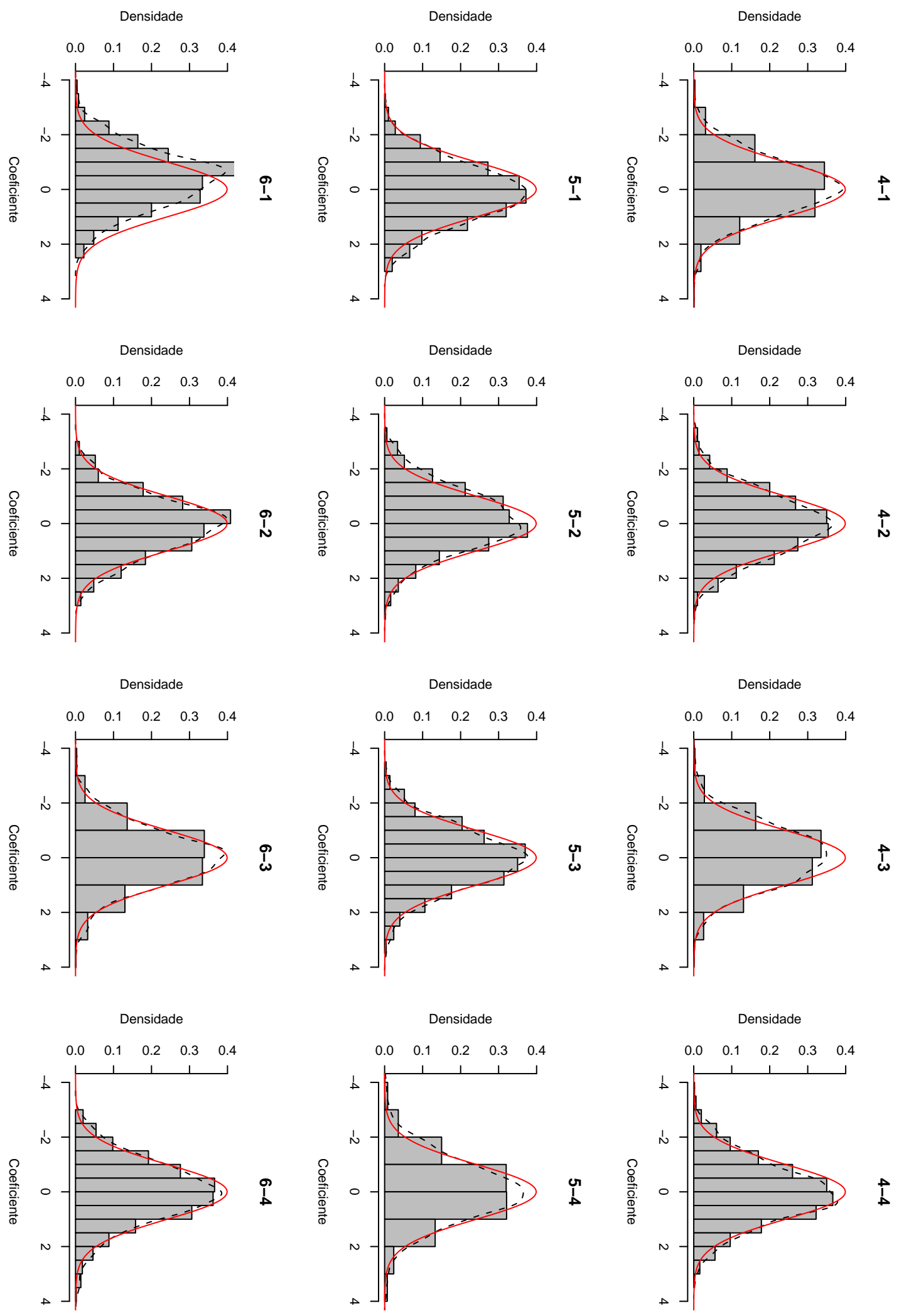

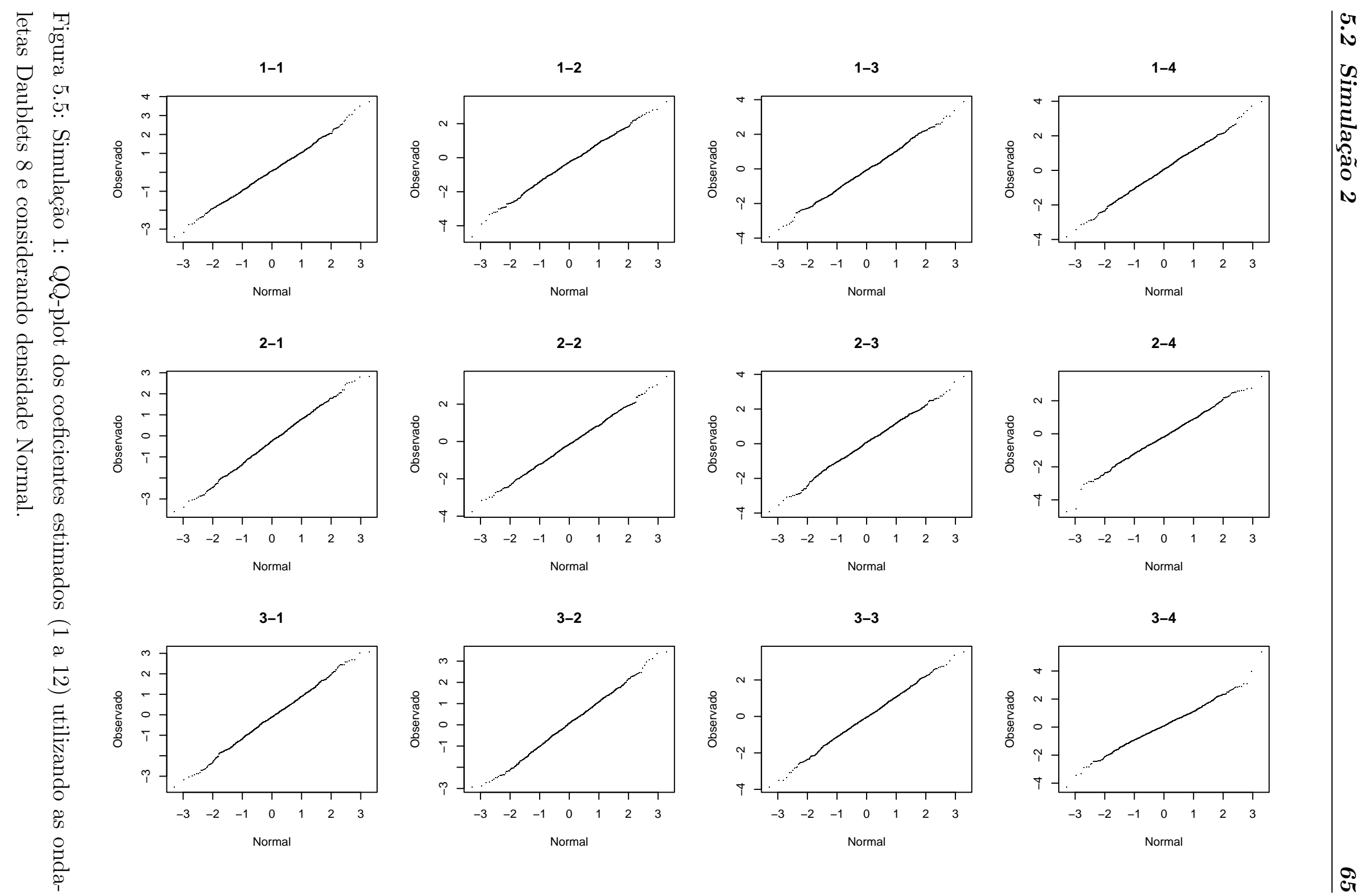

3-2

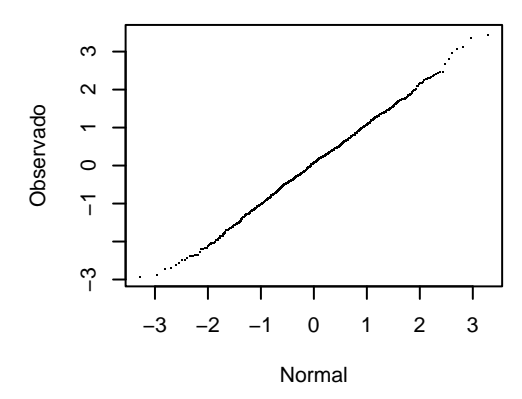

3-3
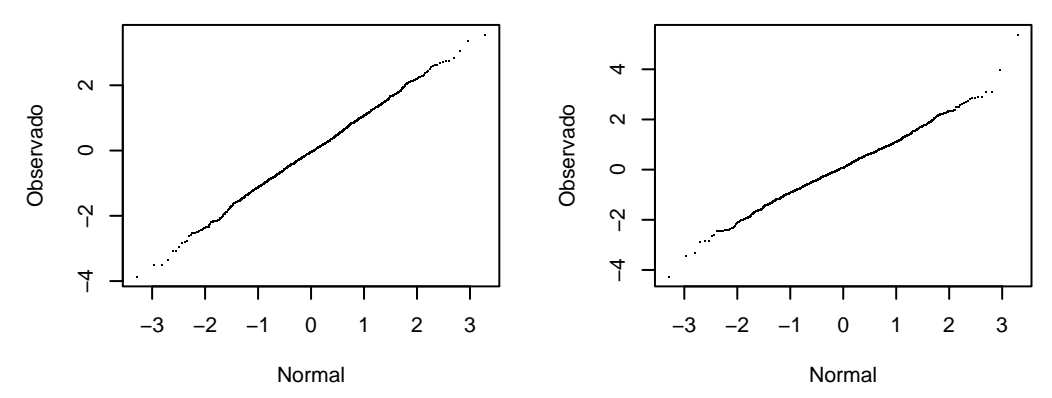

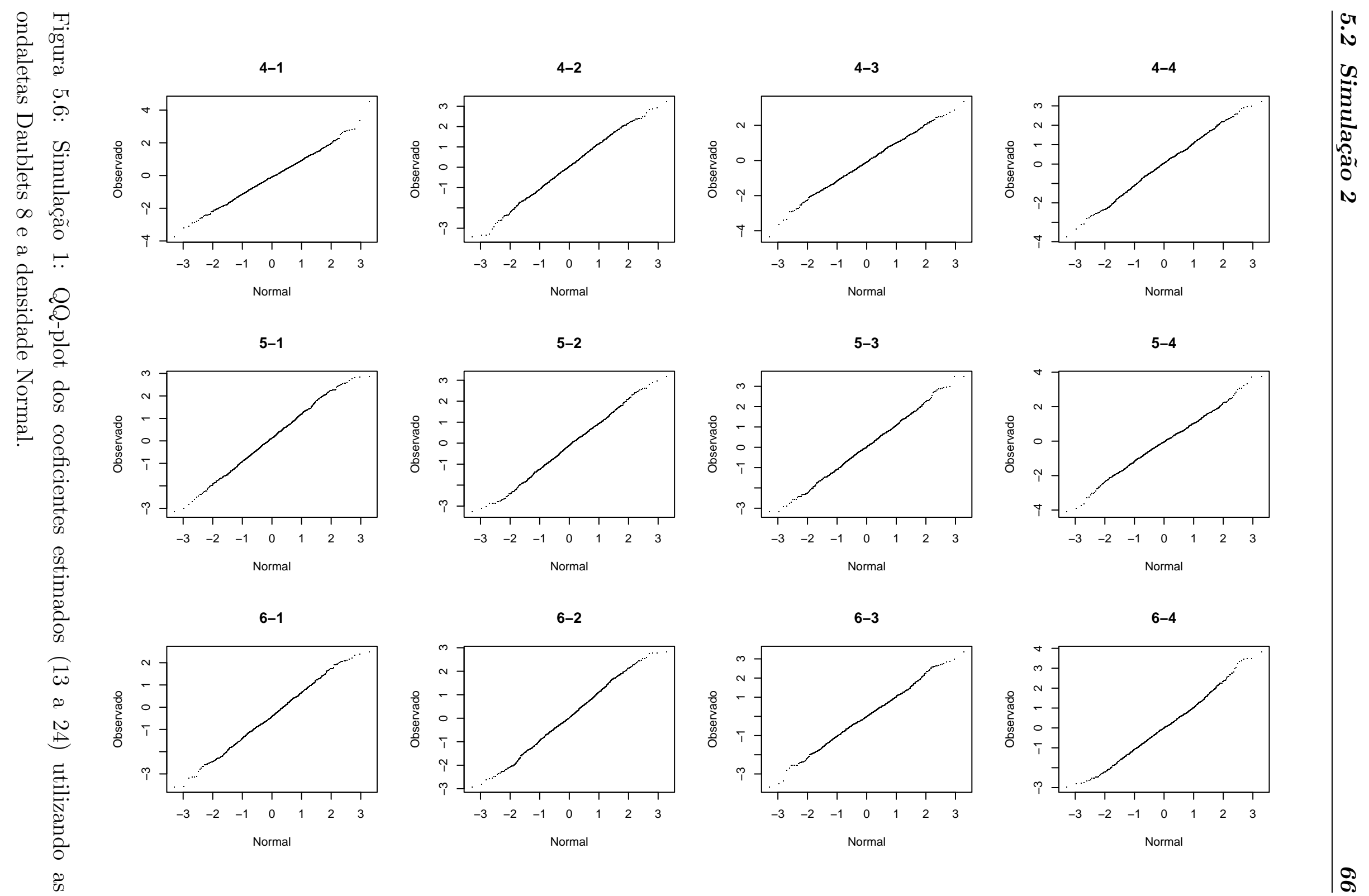

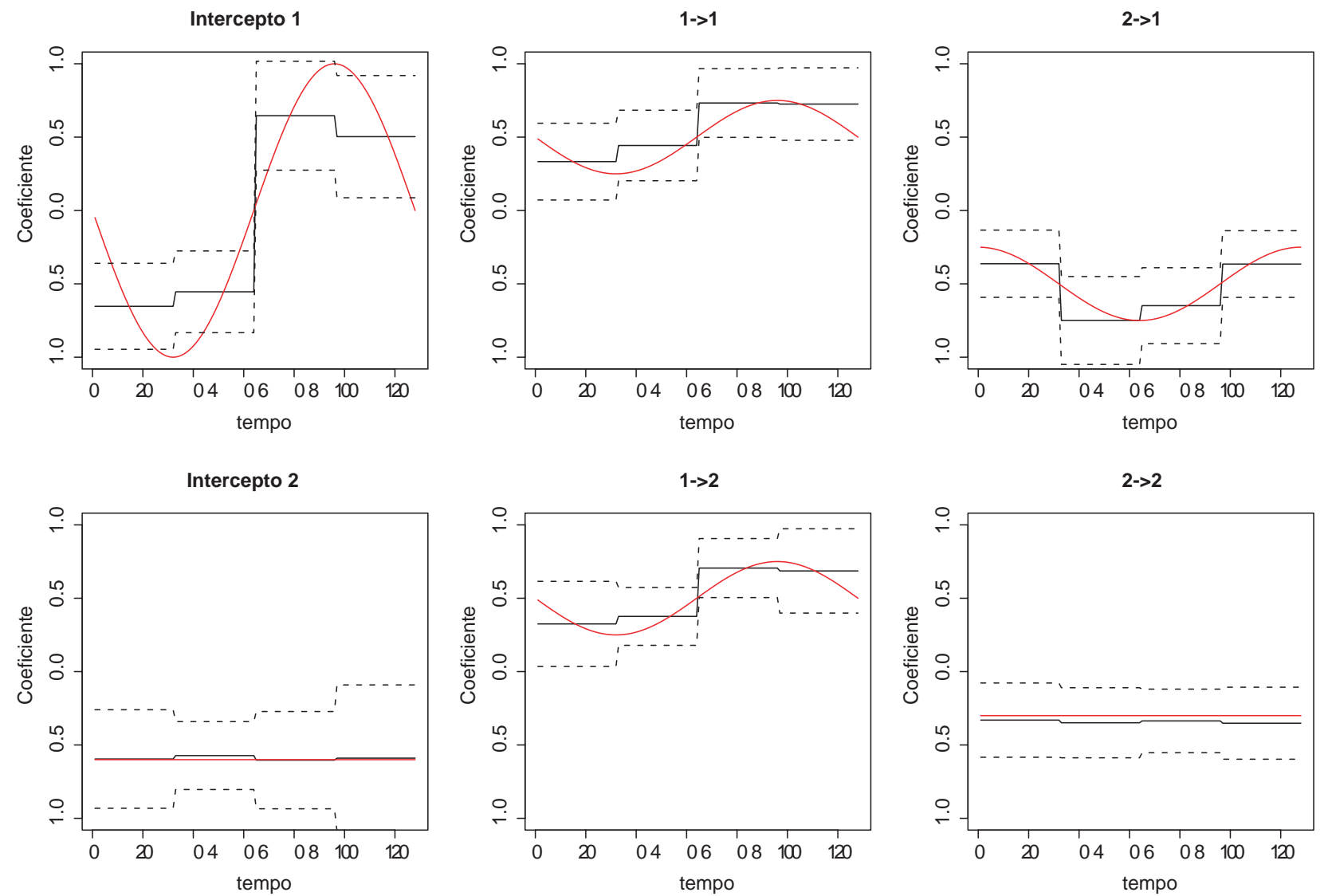

Figura 5.7: Simulação 1: Coeficientes do modelo DVAR utilizando as ondaletas de Haar. A linha vermelha e preta indicam os valores das curvas verdadeiras e esperanças estimadas, respectivamente. As linhas tracejadas indicam um intervalo de 1 erro padrão. 

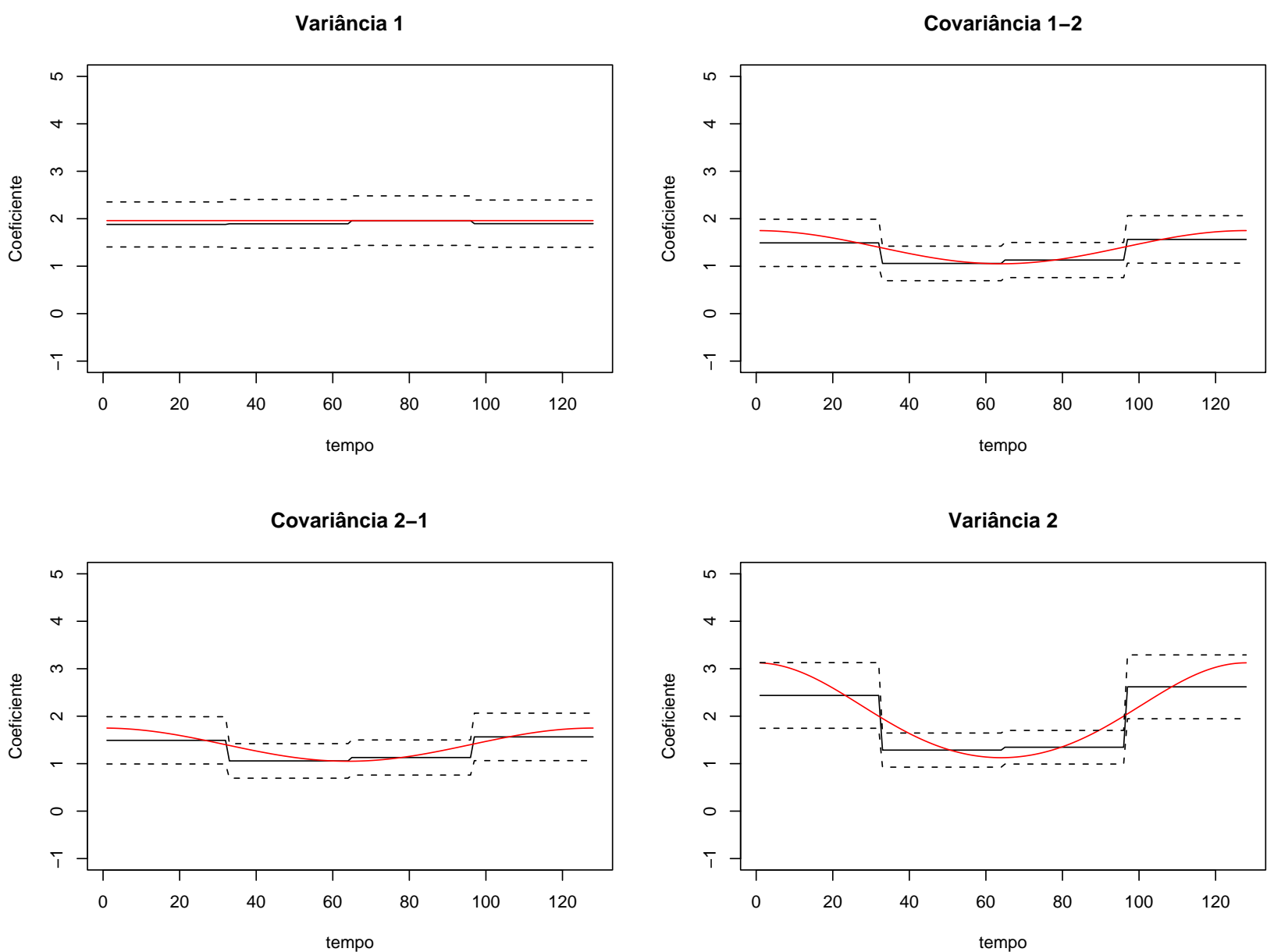

Figura 5.8: Simulação 1: Matriz de Covariância dos Erros do modelo DVAR utilizando as ondaletas de Haar. A linha vermelha e preta indicam os valores das curvas verdadeiras e esperanças estimadas, respectivamente. As linhas tracejadas indicam um intervalo de 1 erro padrão. 


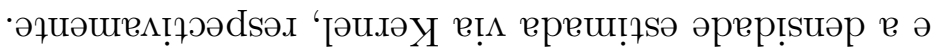

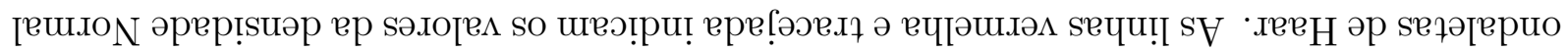

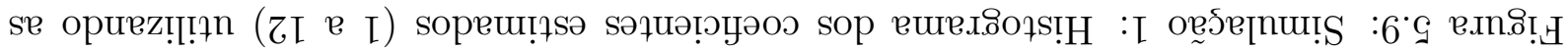
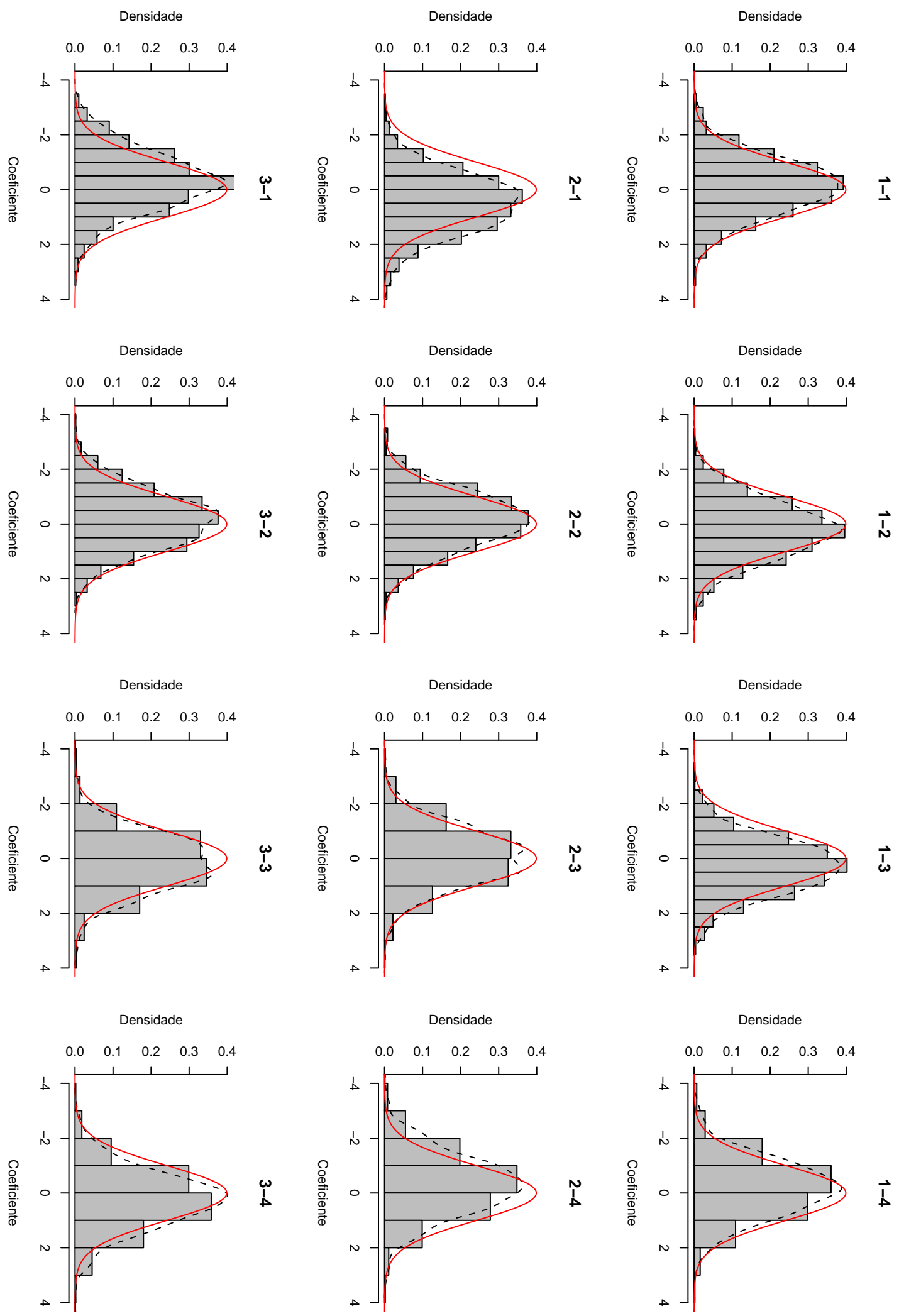


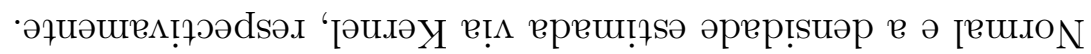

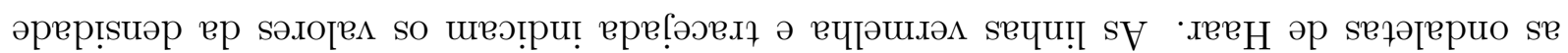

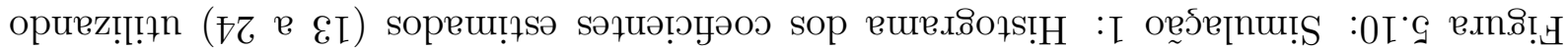
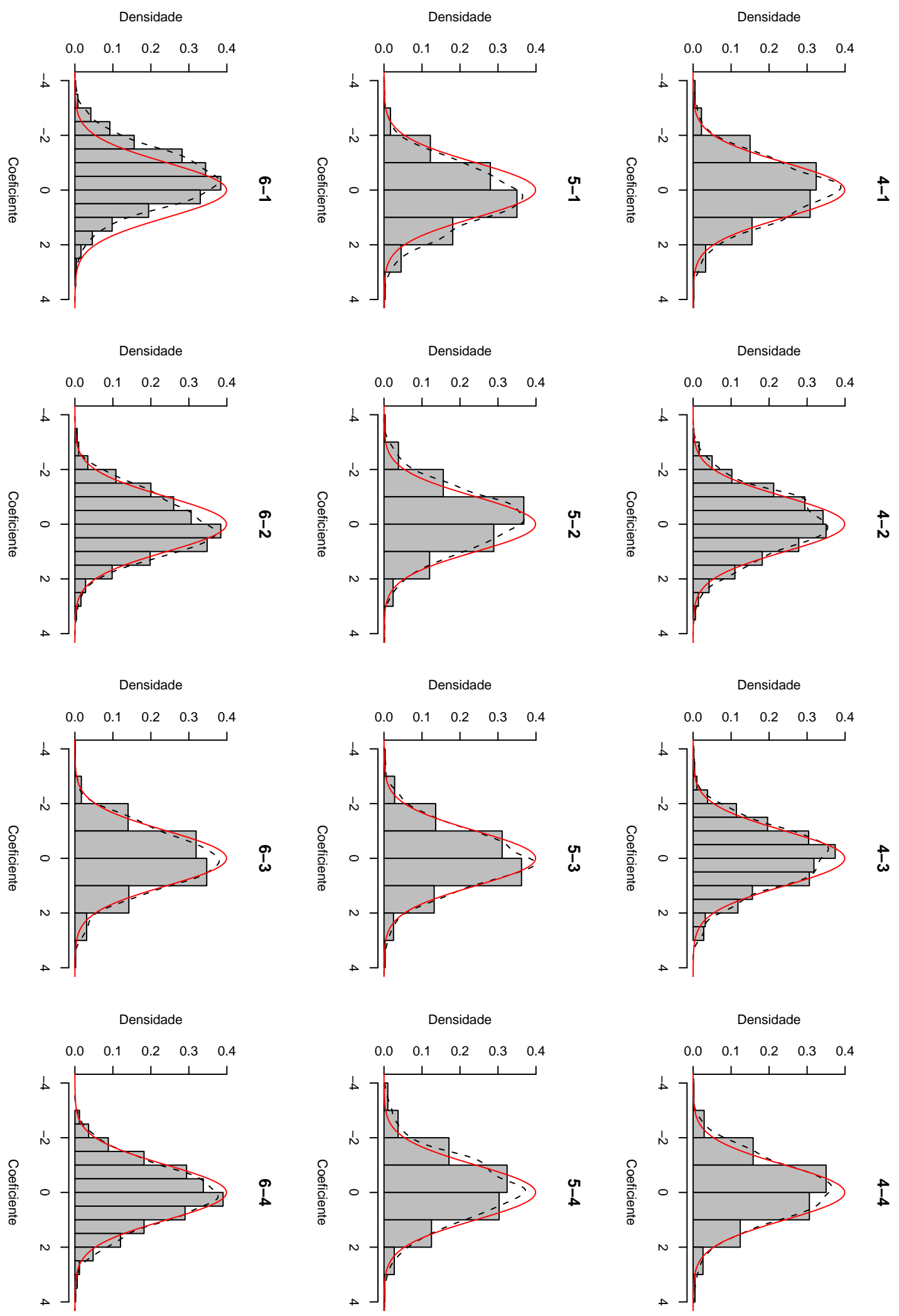

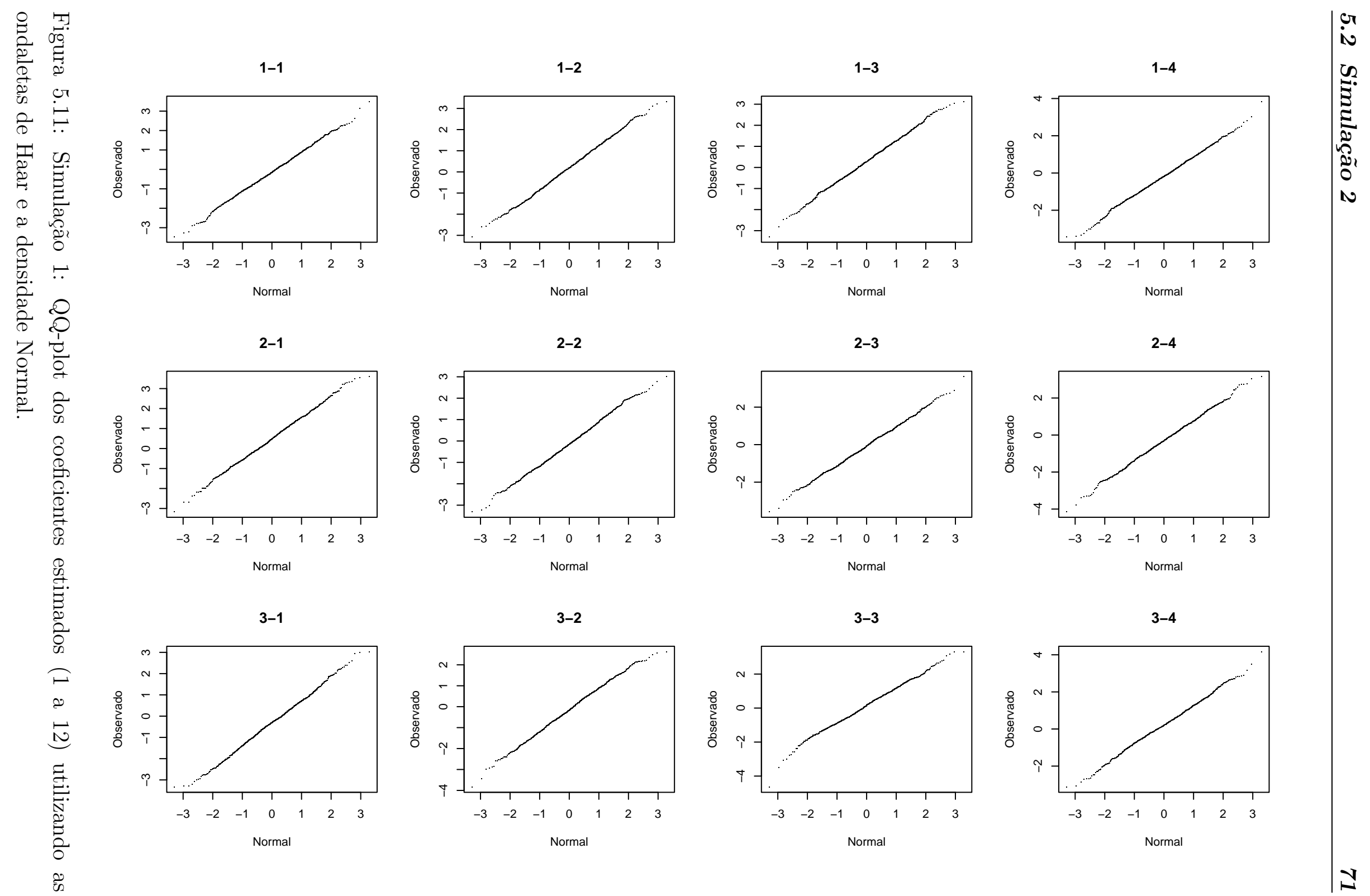

3-2

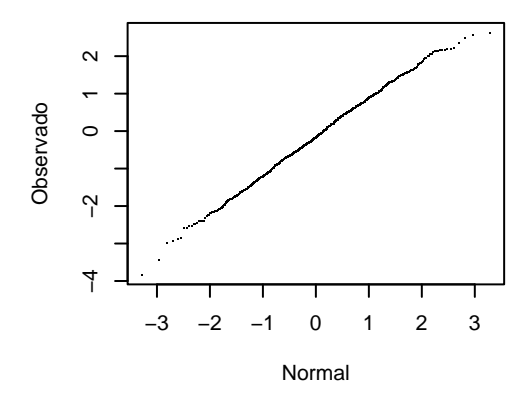

3-3
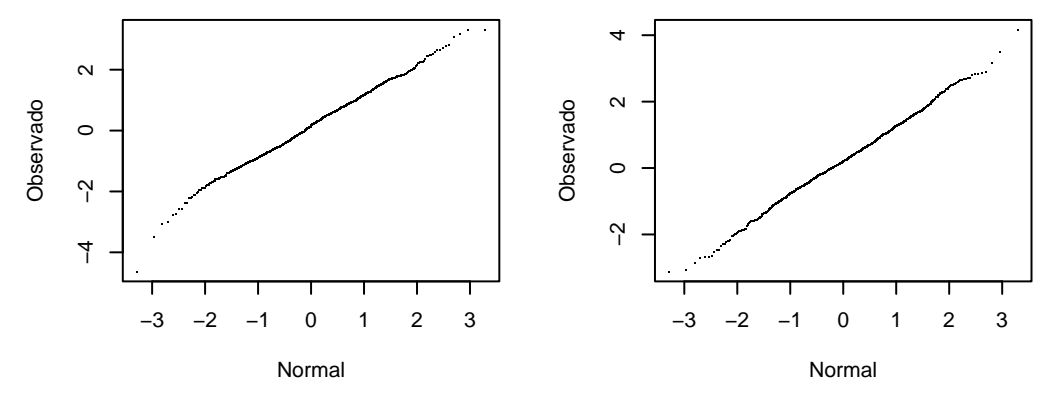

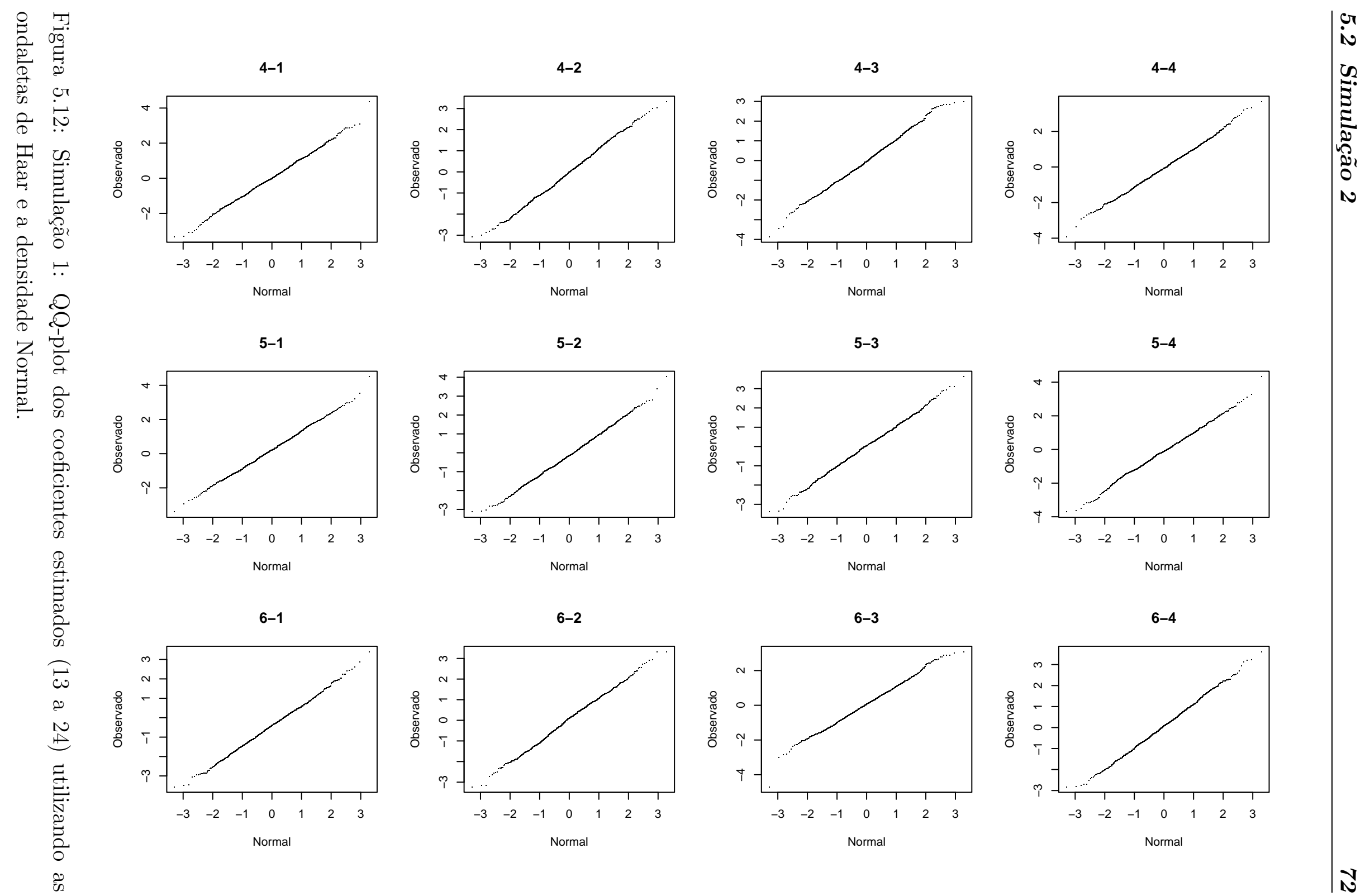

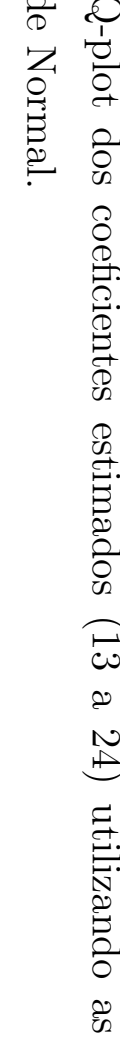
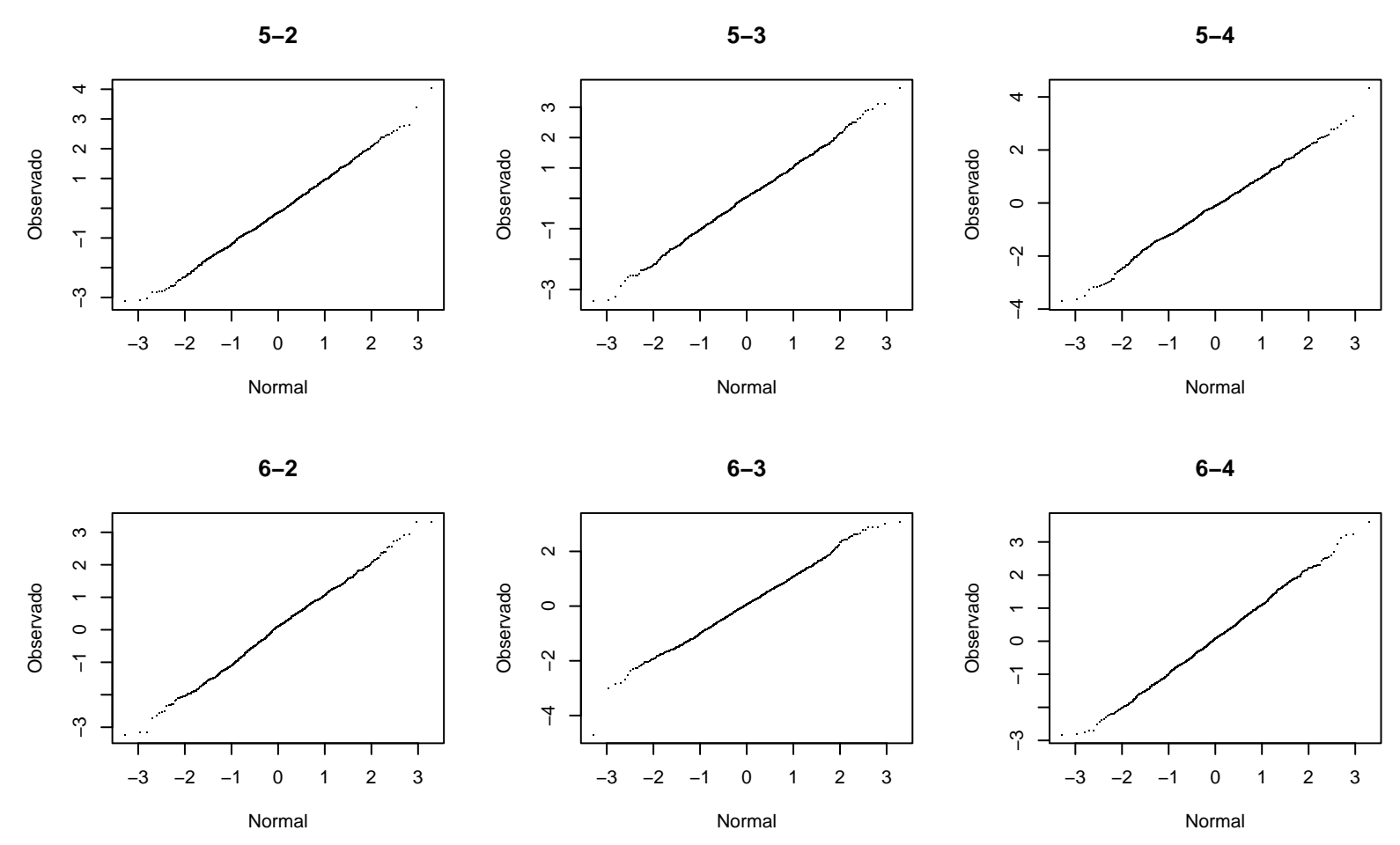
$H_{A}$ : O coeficiente de causalidade varia no tempo;

pode ser especificado na forma

$H_{0}: a_{j, k}=0$ para todo $j=1,2, \ldots$ e $k=1,2, \ldots, 2^{j-1}$

$H_{A}$ : Existe pelo menos um coeficiente não nulo.

Este teste pode ser realizado utilizando o teste de Wald para contrastes, descrito no capítulo 3. Assim sendo, considere um processo DVAR bivariado dado por

$$
x_{t}=u(t)+\mathbf{A}(t) x_{t-1}+\epsilon_{t},
$$

onde

$$
\mathbf{u}(t)=\left(\begin{array}{c}
\operatorname{sen}\left(\frac{2 \pi t}{128}+\pi\right) \\
-0,6
\end{array}\right), \mathbf{A}(t)=\left(\begin{array}{cc}
0,5-\frac{\operatorname{sen}\left(\frac{2 \pi t}{160}\right)}{4} & -0,5+\frac{\cos \left(\frac{2 \pi t}{128}\right)}{4} \\
\theta \frac{\operatorname{sen}\left(\frac{2 \pi t}{128}\right)}{4} & -0,3
\end{array}\right)
$$

e

$$
\boldsymbol{\Sigma}(t)=\left(\begin{array}{cc}
1,4 & 1,4\left(1+\frac{\cos \left(\frac{2 \pi t}{128}\right)}{4}\right) \\
1,4\left(1+\frac{\cos \left(\frac{2 \pi t}{128}\right)}{4}\right) & 2\left(1+\frac{\cos \left(\frac{2 \pi t}{128}\right)}{4}\right)^{2}
\end{array}\right)
$$

onde $u(t)$ é o vetor de interceptos, $\mathbf{A}(t)$ é a matriz de coeficientes autoregressivos e $\boldsymbol{\Sigma}(t)$ é a matriz de covariância dos erros aleatórios. Note que o coeficiente de causalidade da primeira série em relação à segunda, depende de um coeficiente $\theta$. Além disso, quanto maior o valor de $\theta$ maior será a variação temporal da causalidade. Assim sendo, simulamos este processo 500 vezes e verificamos a proporção de rejeição do teste utilizando as ondaletas de Haar e Daublets 8. Além disso, para estimar a função poder, consideramos os valores $(0 ; 0,2 ; 0,4 ; 0,6 ; 0,8$ e 1,0) para o coeficiente $\theta$ e $(T=64,128$ e 256) para extensão de série. O truncamento das funções de base foi feita na escala 2 , resultando em quatro ondaletas. Os resultados são apresentados nas Tabelas 5.1, 5.2 e nas Figuras 5.13 e 5.14.

\subsection{Simulação 3}

Neste conjunto de simulações, desejamos avaliar o desempenho dos estimadores dos parâmetros do modelo DVAR, nos casos nos quais a função intercepto e as funções de causalidade não são suaves no tempo, mas funções quadradas. Dessa forma, considerou-se duas $f(t)$ e $g(t)$ onde,

$$
f(t)= \begin{cases}1, & \text { se } \frac{T}{2}<t \leq T \\ 0, & \text { caso contrário }\end{cases}
$$




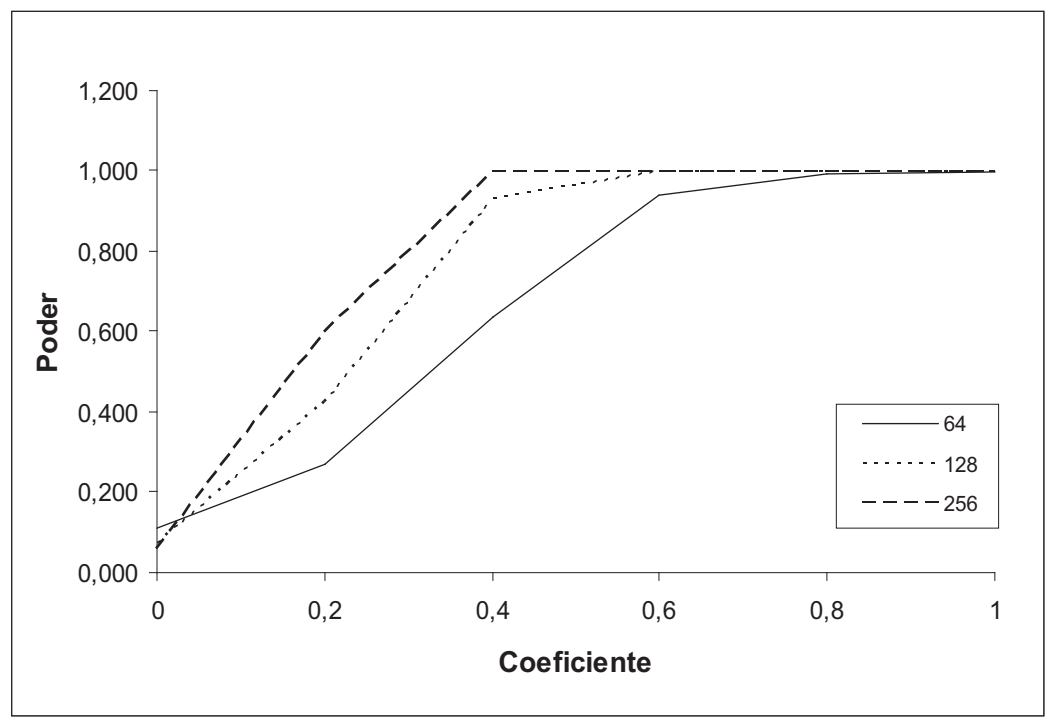

Figura 5.13: Simulação 2: Poder do teste de coeficiente variando no tempo utilizando a ondaleta D8.

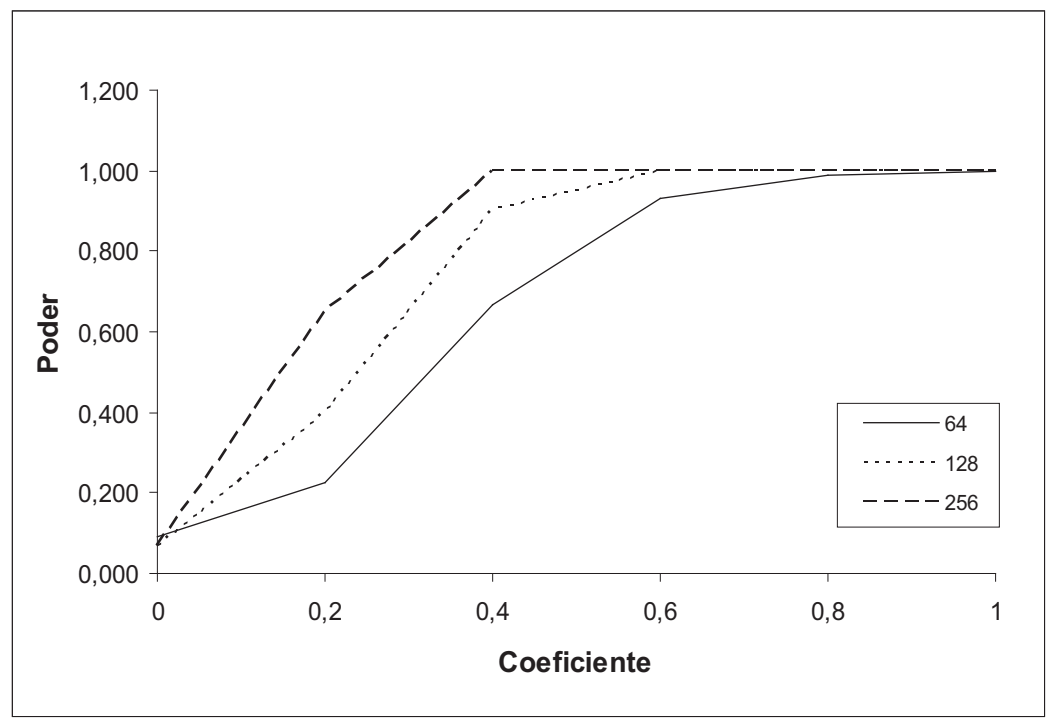

Figura 5.14: Simulação 2: Poder do teste de coeficiente variando no tempo utilizando a ondaleta de Haar. 


\begin{tabular}{|c|c|cccccc|}
\hline Ondaleta & $\mathrm{T}$ & \multicolumn{7}{|c|}{$\theta$} \\
\hline \hline \multirow{3}{*}{ D8 } & & 0 & 0,2 & 0,4 & 0,6 & 0,8 & 1 \\
& 64 & 0,112 & 0,270 & 0,634 & 0,942 & 0,992 & 1,000 \\
& 128 & 0,074 & 0,426 & 0,932 & 1,000 & 1,000 & 1,000 \\
& 256 & 0,056 & 0,596 & 1,000 & 1,000 & 1,000 & 1,000 \\
\hline \multirow{3}{*}{ Haar } & 64 & 0,090 & 0,226 & 0,668 & 0,930 & 0,988 & 0,998 \\
& 128 & 0,068 & 0,398 & 0,902 & 0,998 & 1,000 & 1,000 \\
& 256 & 0,066 & 0,650 & 0,998 & 1,000 & 1,000 & 1,000 \\
\hline
\end{tabular}

Tabela 5.1: Simulação 2: Proporção de Rejeição para o teste de funções de causalidade variando no tempo.

e

$$
g(t)= \begin{cases}1, & \text { se } \frac{T}{4}<t<\frac{3 T}{4} \\ 0, & \text { caso contrário }\end{cases}
$$

onde $T$ é a extensão da séries. Note que $f(t)$ é uma função do tipo threshold e $g(t)$ uma função retangular. Assim sendo, consideramos um modelo DVAR dado por

$$
x_{t}=u(t)+\mathbf{A}(t) x_{t-1}+\epsilon_{t},
$$

onde

$$
\mathbf{u}(t)=\left(\begin{array}{c}
0,25 f(t) \\
0,3
\end{array}\right), \mathbf{A}(t)=\left(\begin{array}{cc}
-0,3 g(t) & -0,4 g(t) \\
0,2 f(t) & -0,3
\end{array}\right)
$$

e

$$
\boldsymbol{\Sigma}(t)=\left(\begin{array}{cc}
0,5+0,5 f(t) & (0,5+0,5 f(t))(0,5+0,5 g(t)) \\
(0,5+0,5 f(t))(0,5+0,5 g(t)) & 2(0,5+0,5 g(t))
\end{array}\right) .
$$

onde $u(t)$ é o vetor de interceptos, $\mathbf{A}(t)$ é a matriz de coeficientes autoregressivos e $\boldsymbol{\Sigma}(t)$ é a matriz de covariância dos erros aleatórios. Realizamos 500 simulações deste processo DVAR, considerando $T=128$ e vetor de erros com distribuição gaussiana multivariada. Para cada simulação obteve-se estimativas dos parâmetros do modelo utilizando o método proposto, considerando-se como funções de base as ondaletas de Haar e D8. Os resultados relativos à viés e variância dos estimadores são apresentados nas Figuras 5.15, 5.16, 5.21 e 5.22, e análises descritivas relativas à distribuição dos estimadores são apresentadas nas Figuras 5.17, 5.18, 5.19, 5.20, 5.23, 5.24, 5.25 e 5.26. 

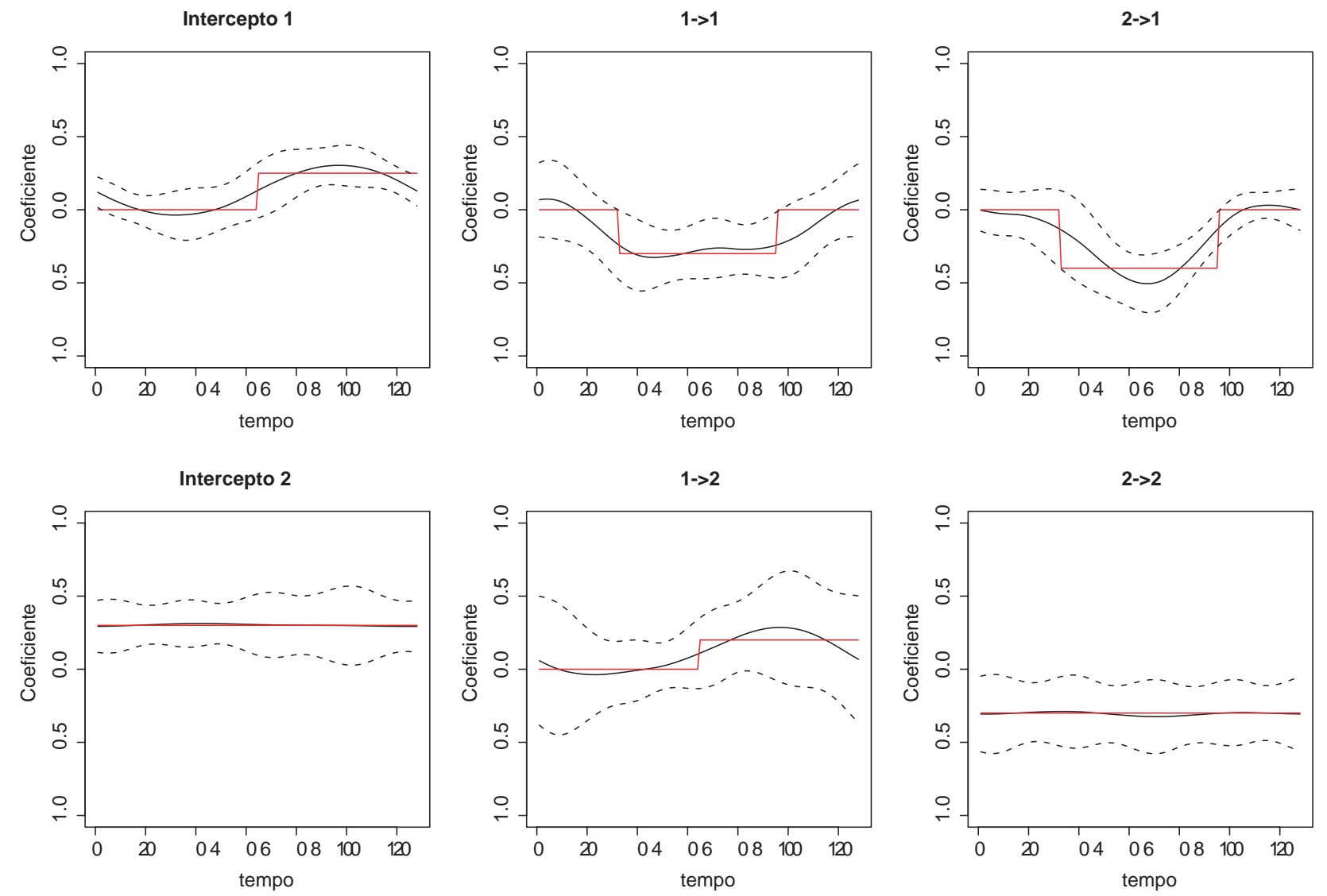

Figura 5.15: Simulação 3: Coeficientes do modelo DVAR utilizando as ondaletas Daublets 8. A linha vermelha e preta indicam os valores das curvas verdadeiras e esperanças estimada, respectivamente. As linhas tracejadas indicam um intervalo de 1 erro padrão. 

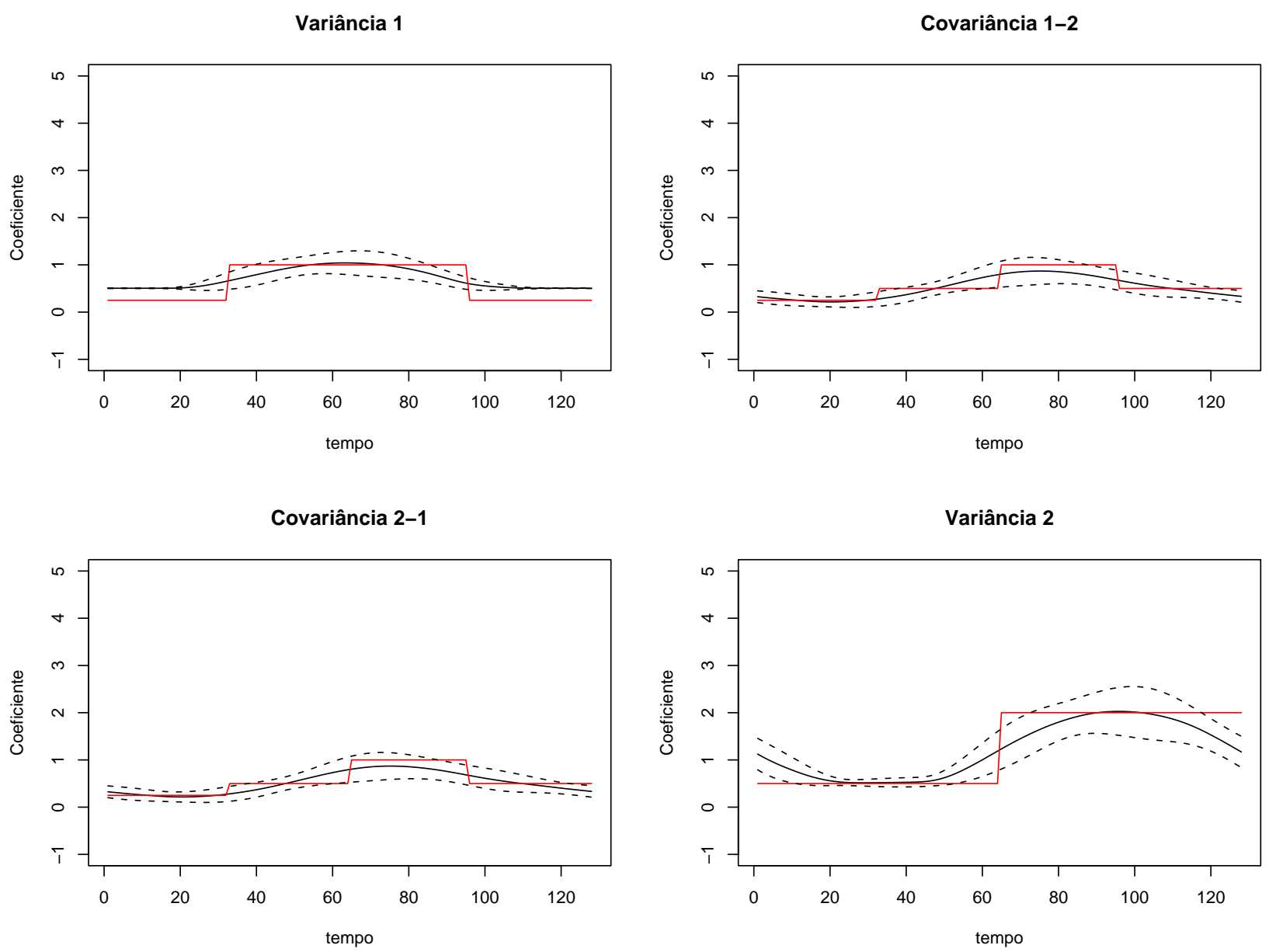

Figura 5.16: Simulação 3: Matriz de Covariância dos Erros do modelo DVAR utilizando as ondaletas Daublets 8. A linha vermelha e preta indicam os valores das curvas verdadeiras e esperanças estimada, respectivamente. As linhas tracejadas indicam um intervalo de 1 erro padrão. 


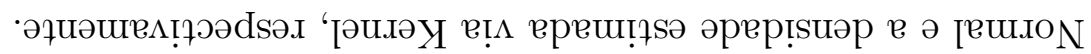

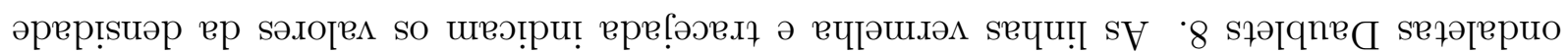

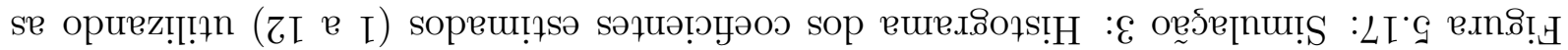
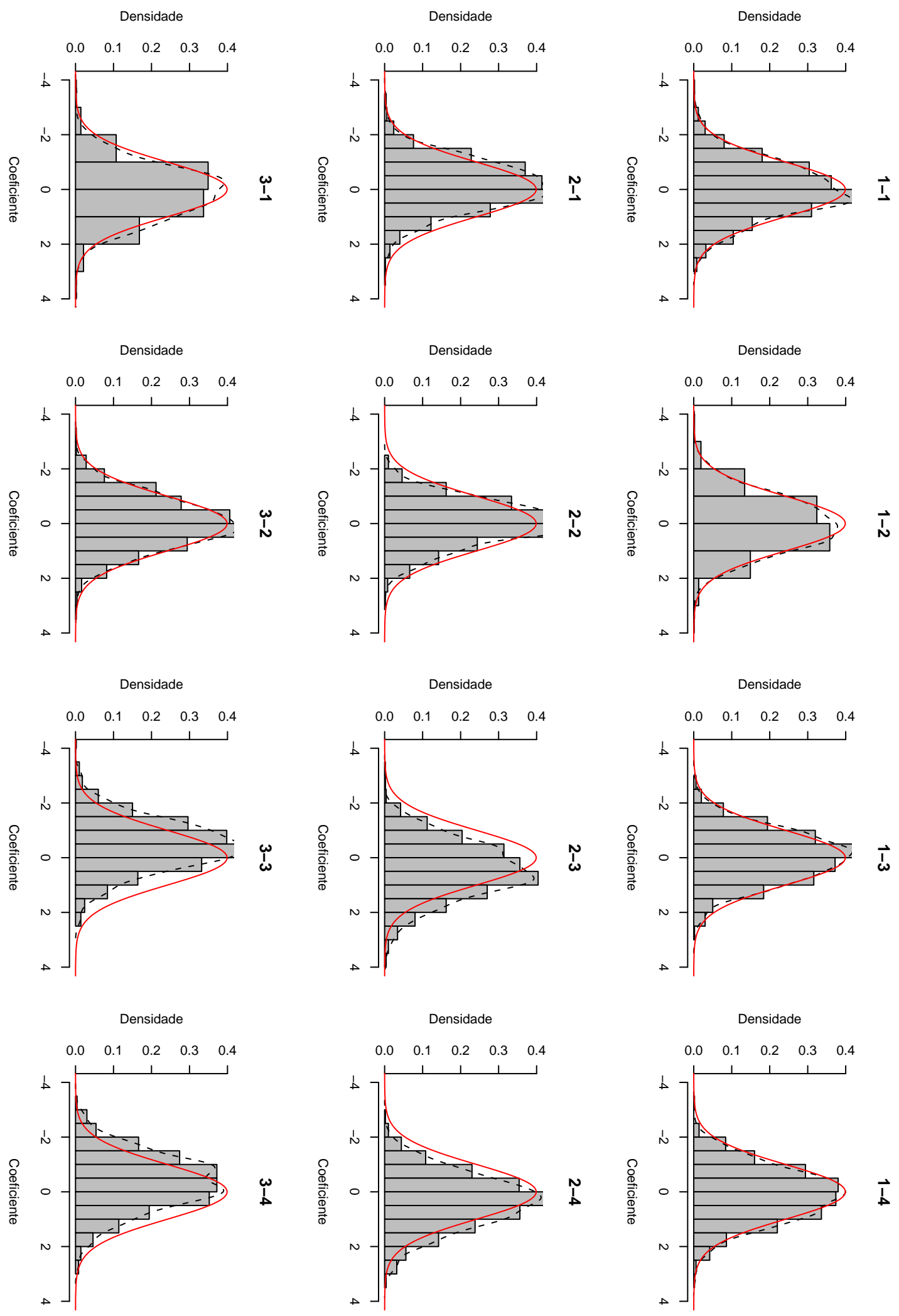


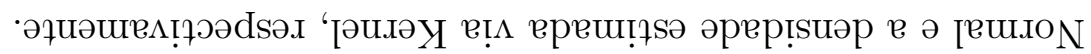

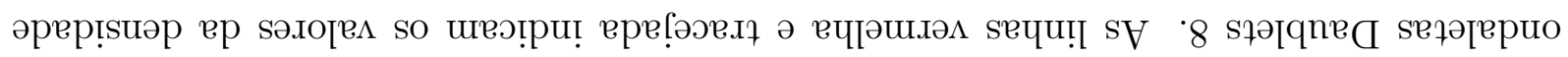

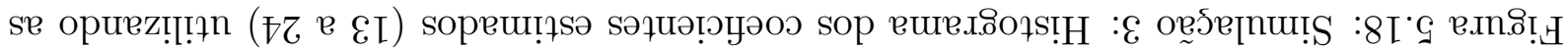
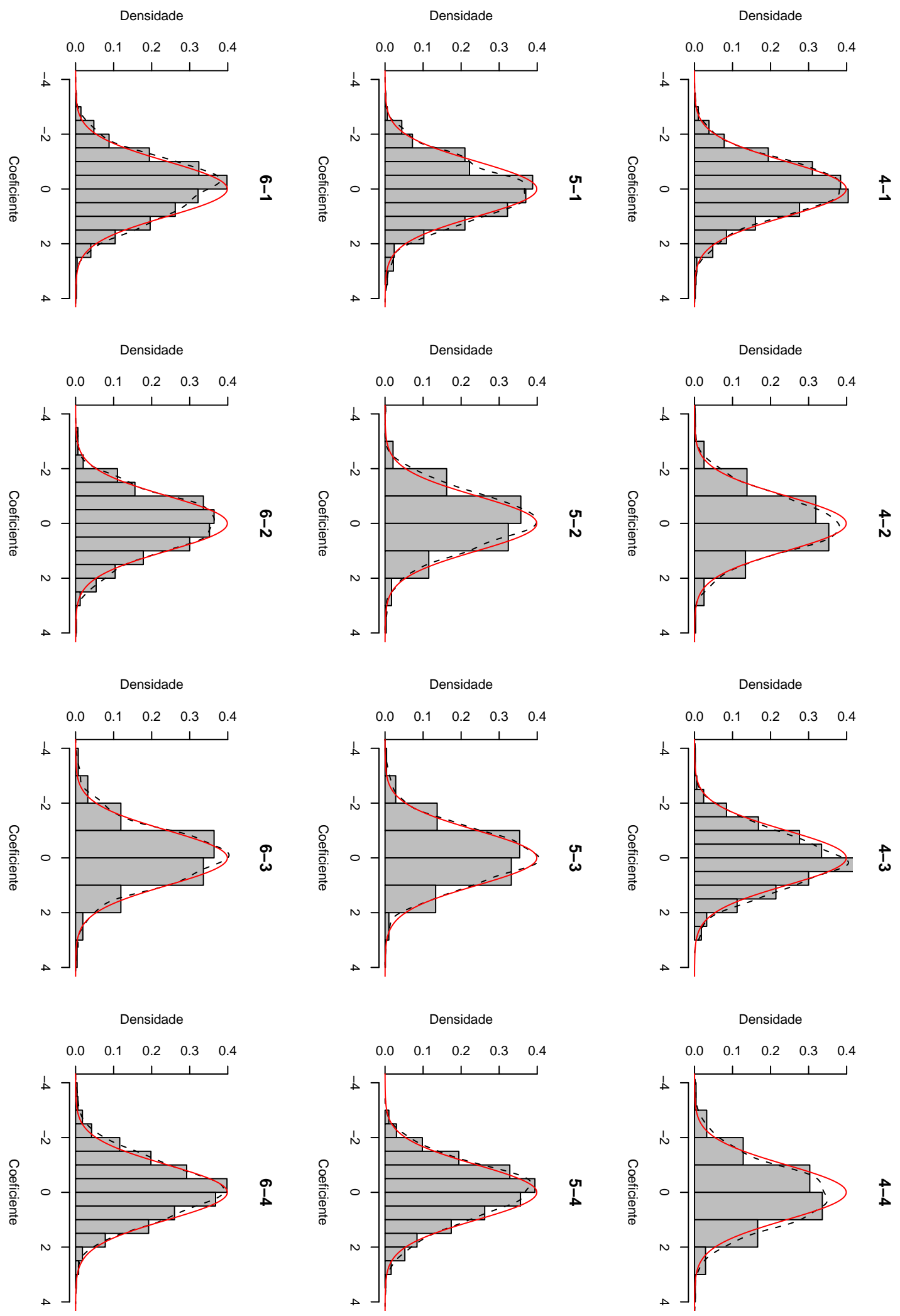

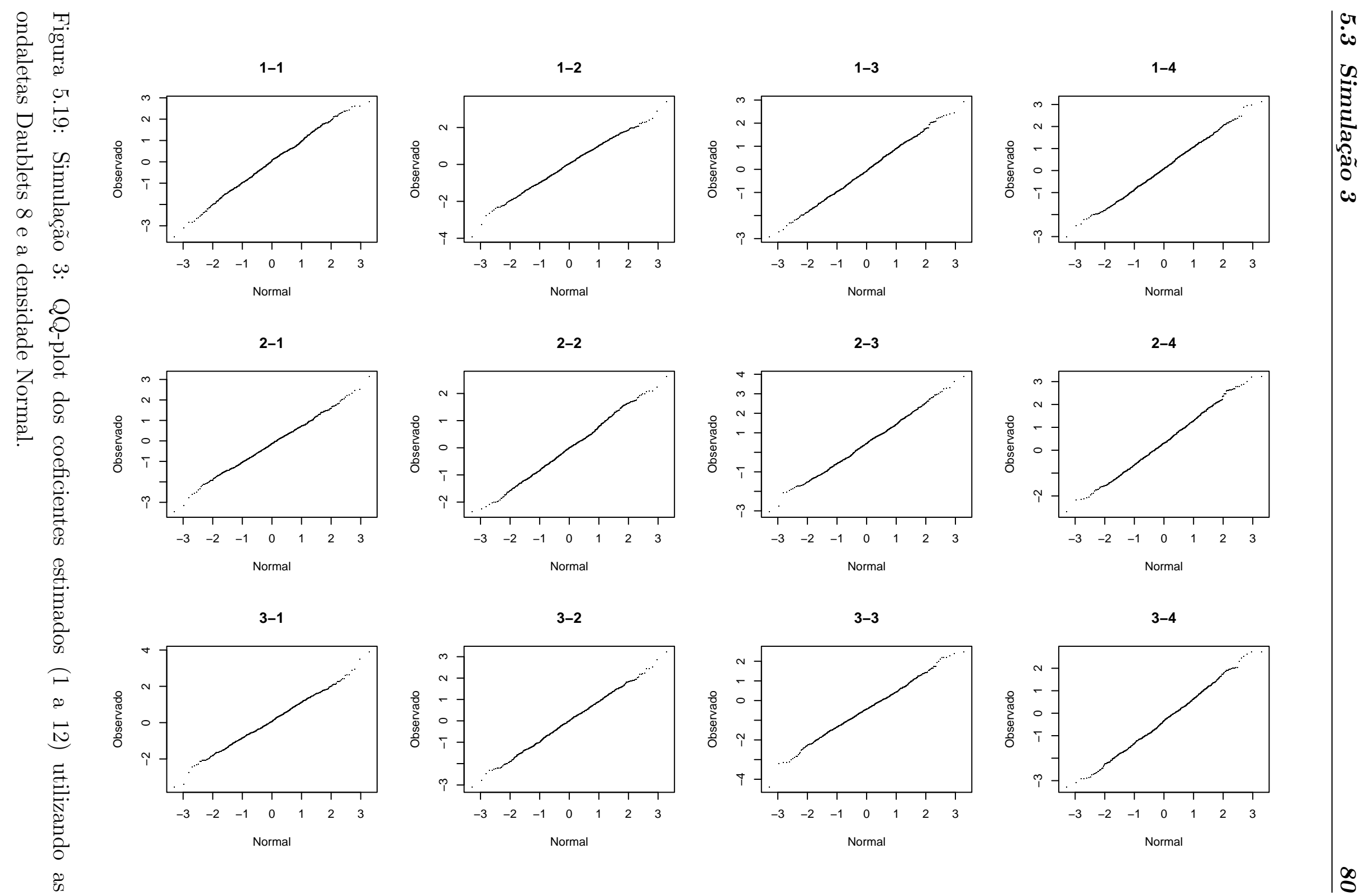

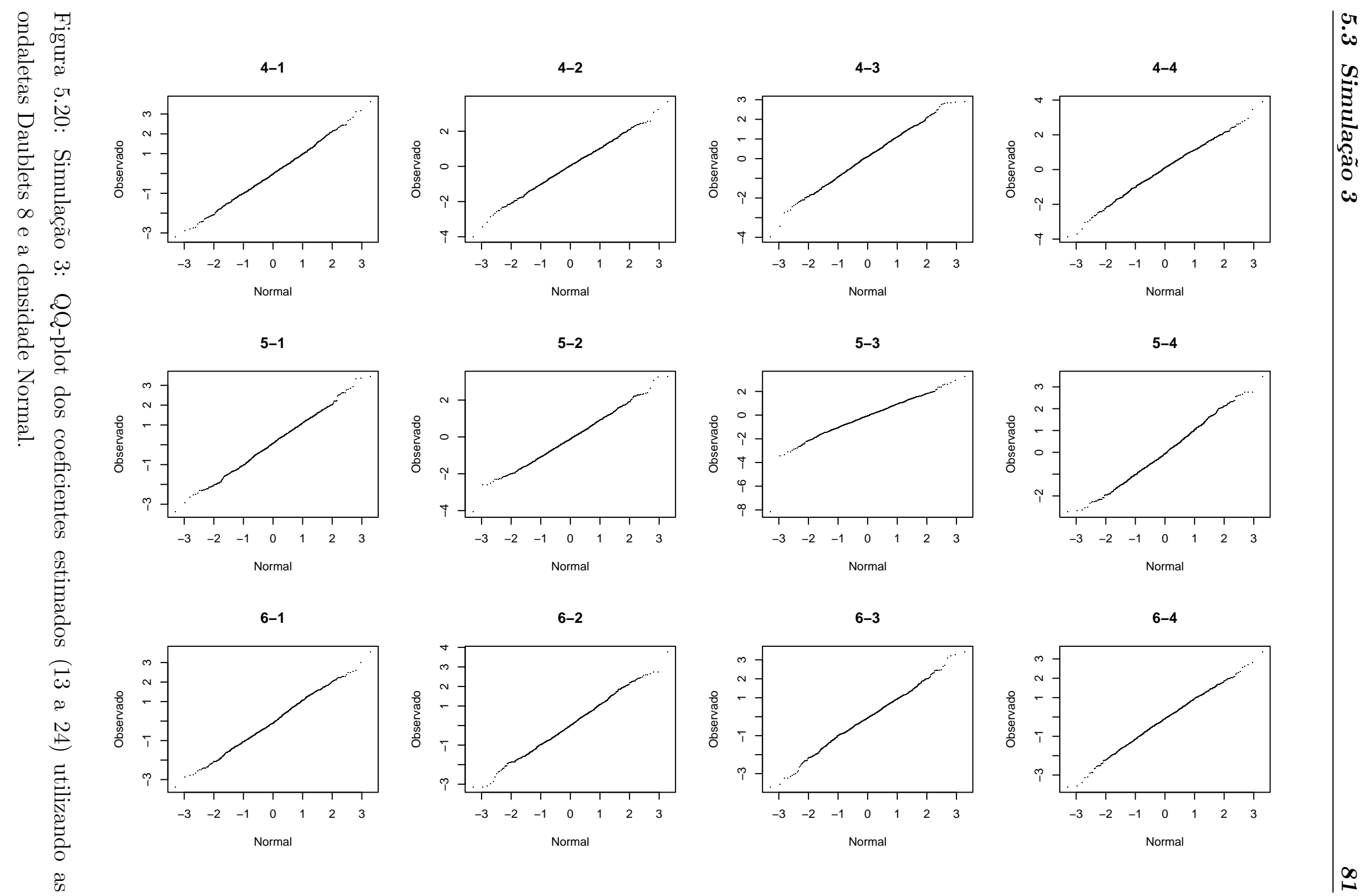

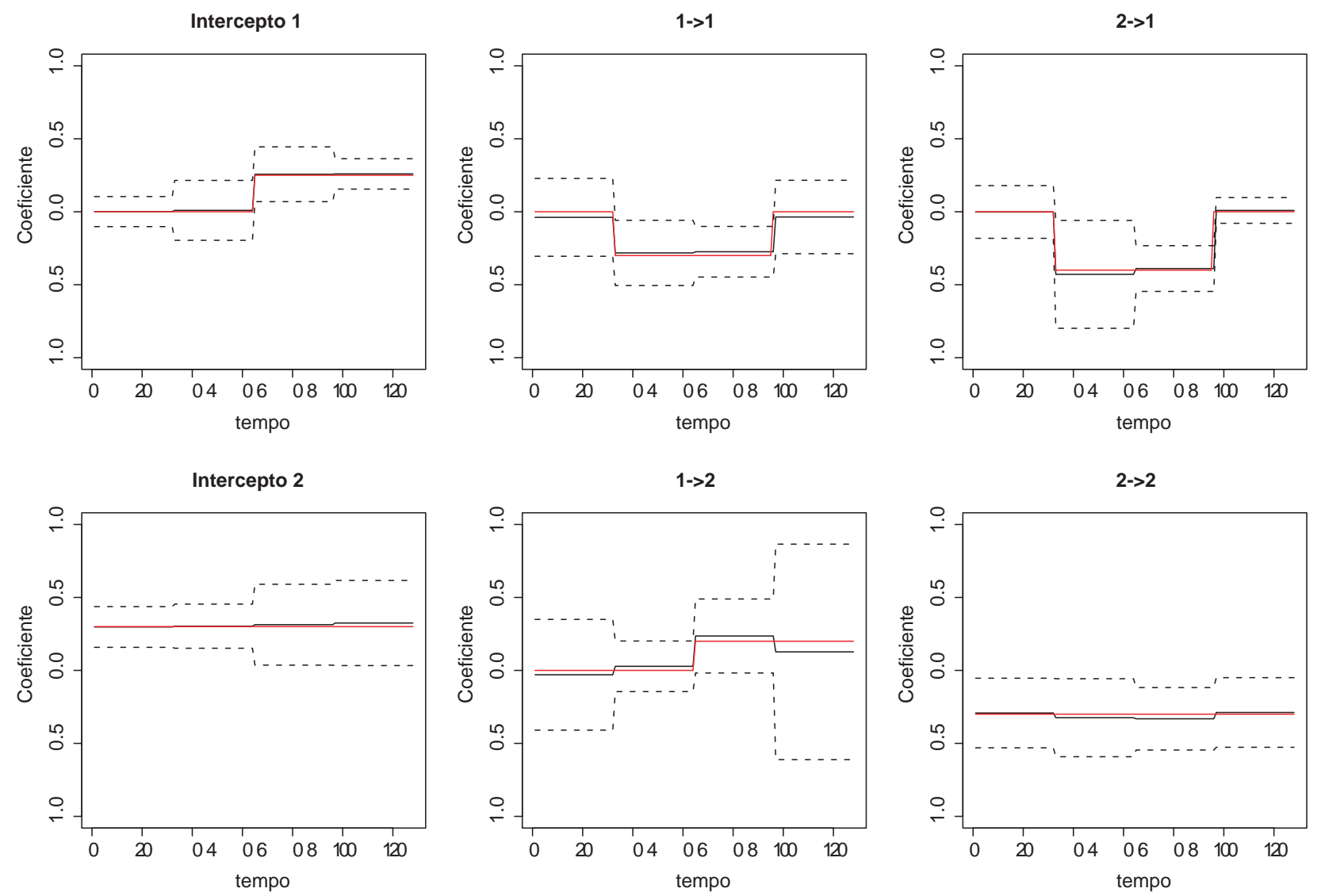

Figura 5.21: Simulação 3: Coeficientes do modelo DVAR utilizando as ondaletas de Haar. A linha vermelha e preta indicam os valores das curvas verdadeiras e esperanças estimada, respectivamente. As linhas tracejadas indicam um intervalo de 1 erro padrão. 

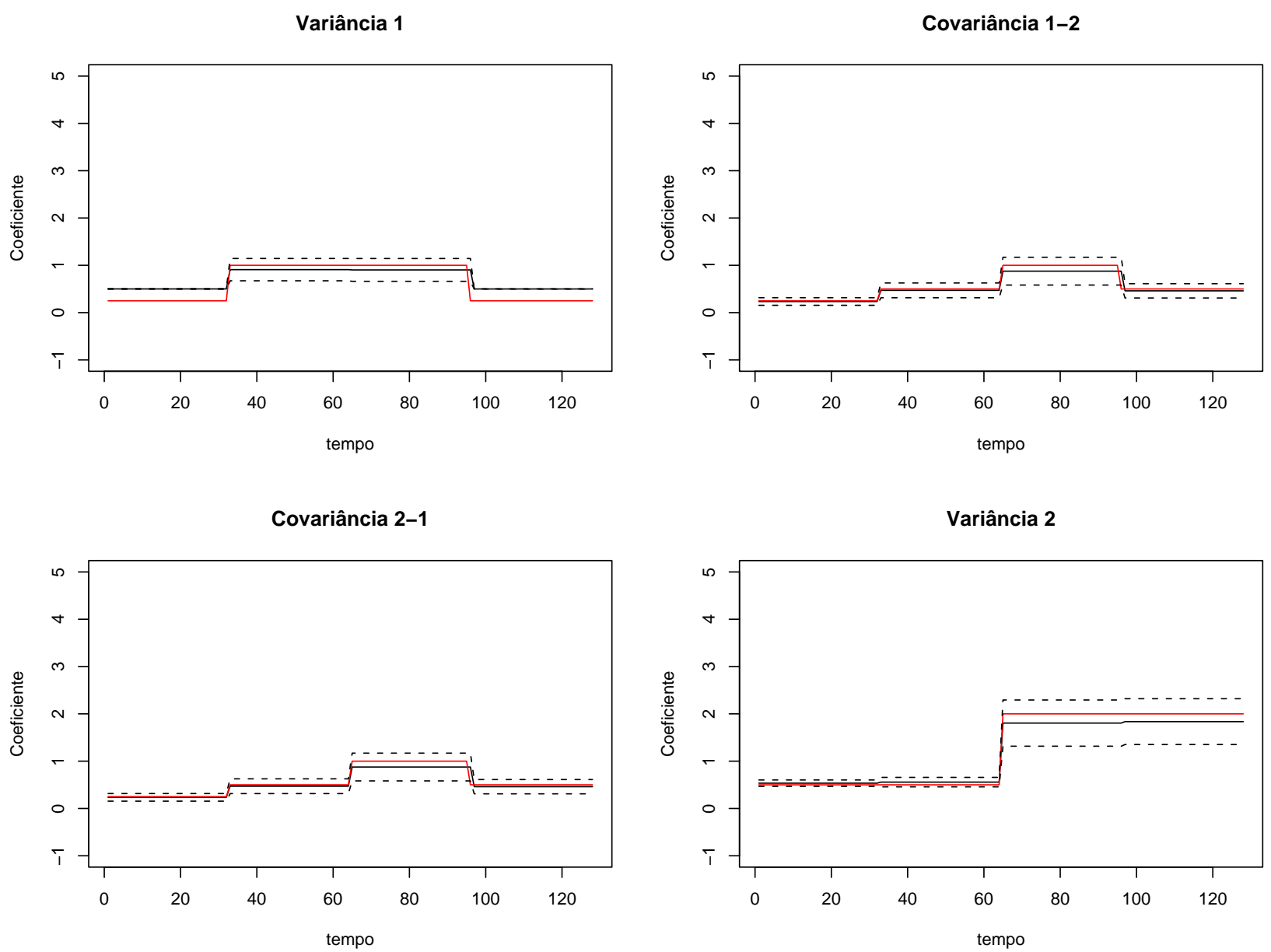

Figura 5.22: Simulação 3: Matriz de Covariância dos Erros do modelo DVAR utilizando as ondaletas de Haar. A linha vermelha e preta indicam os valores das curvas verdadeiras e esperanças estimada, respectivamente. As linhas tracejadas indicam um intervalo de 1 erro padrão. 


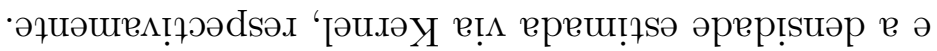

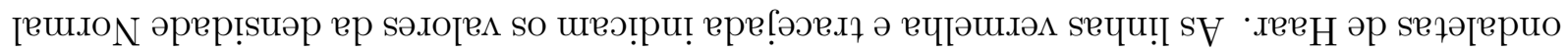

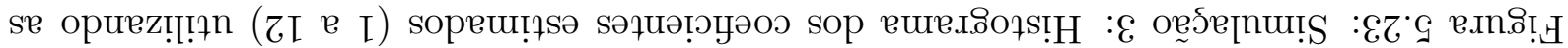
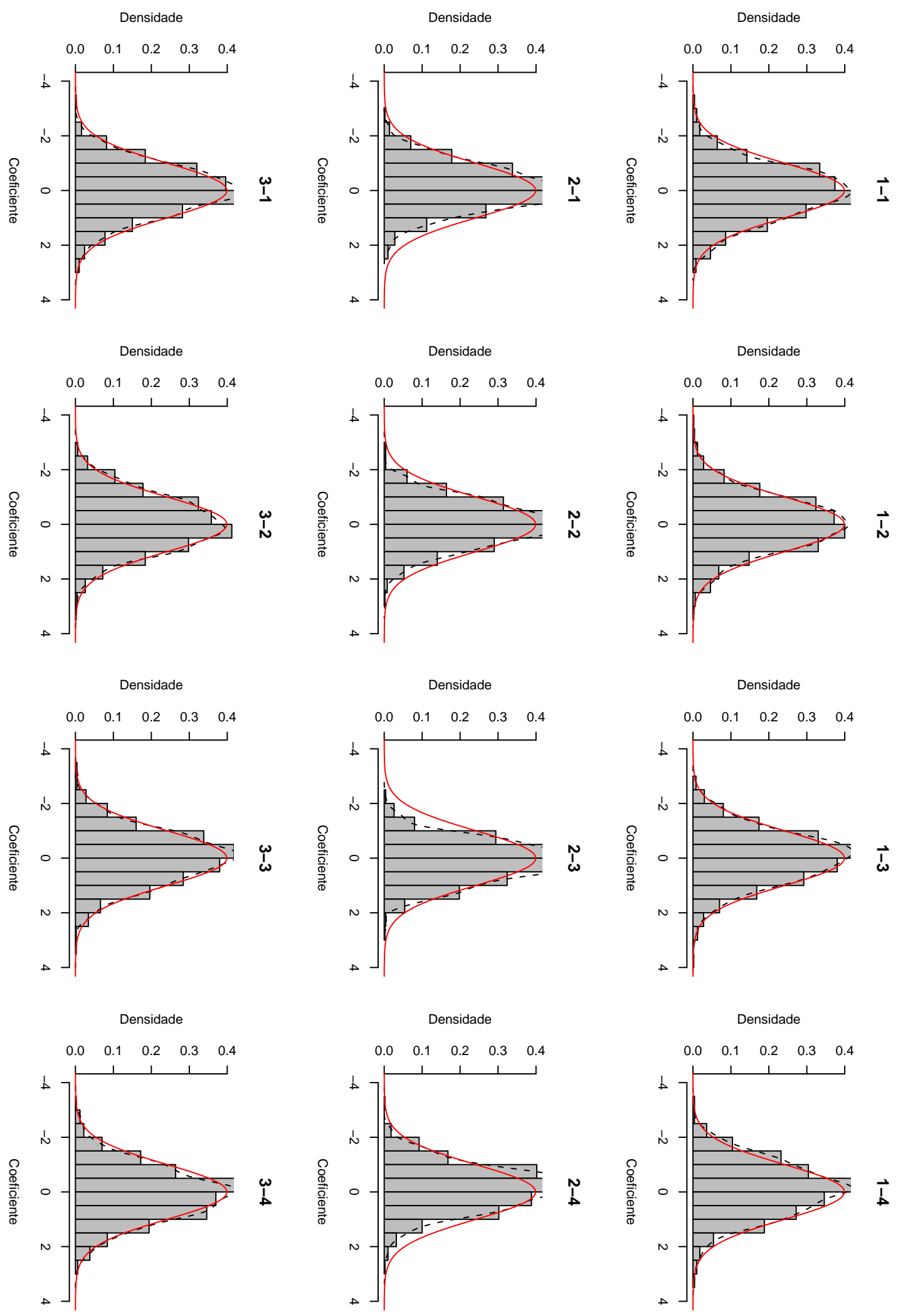


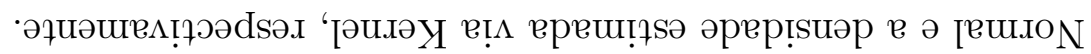

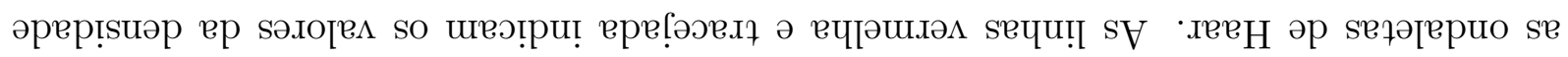

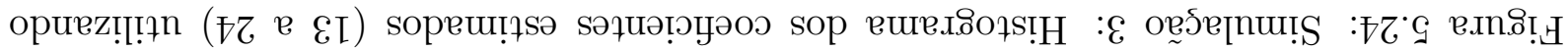
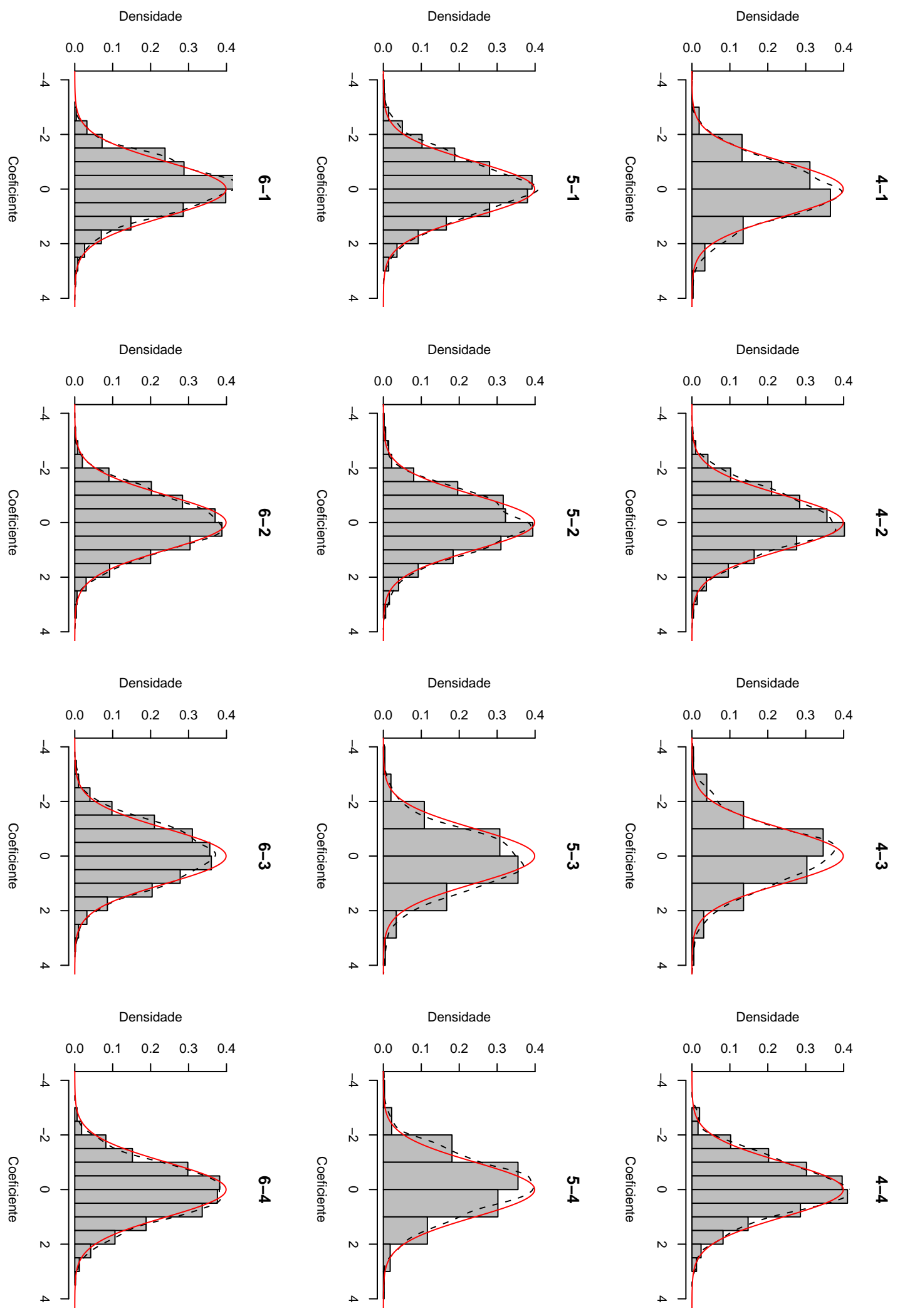

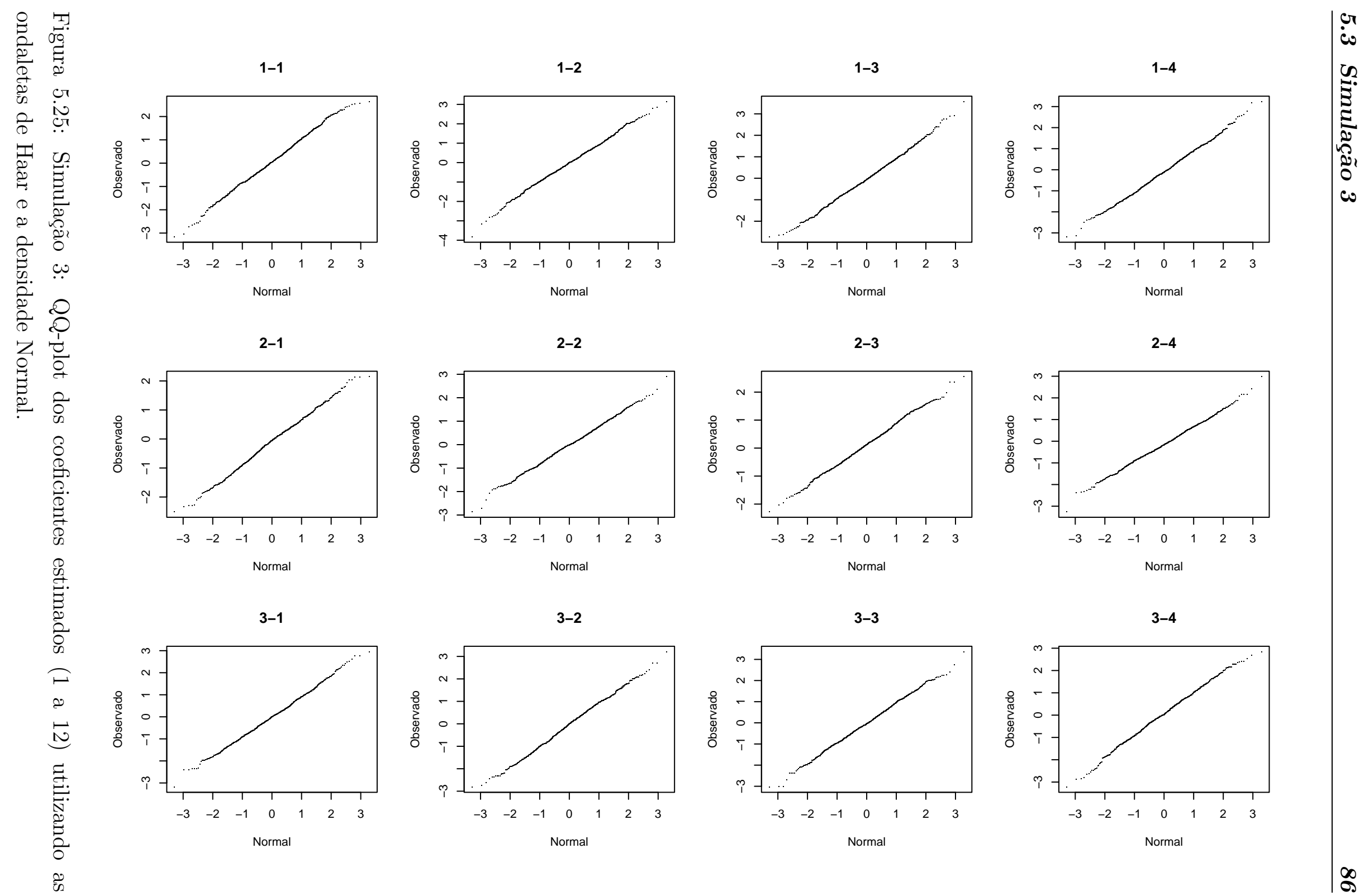

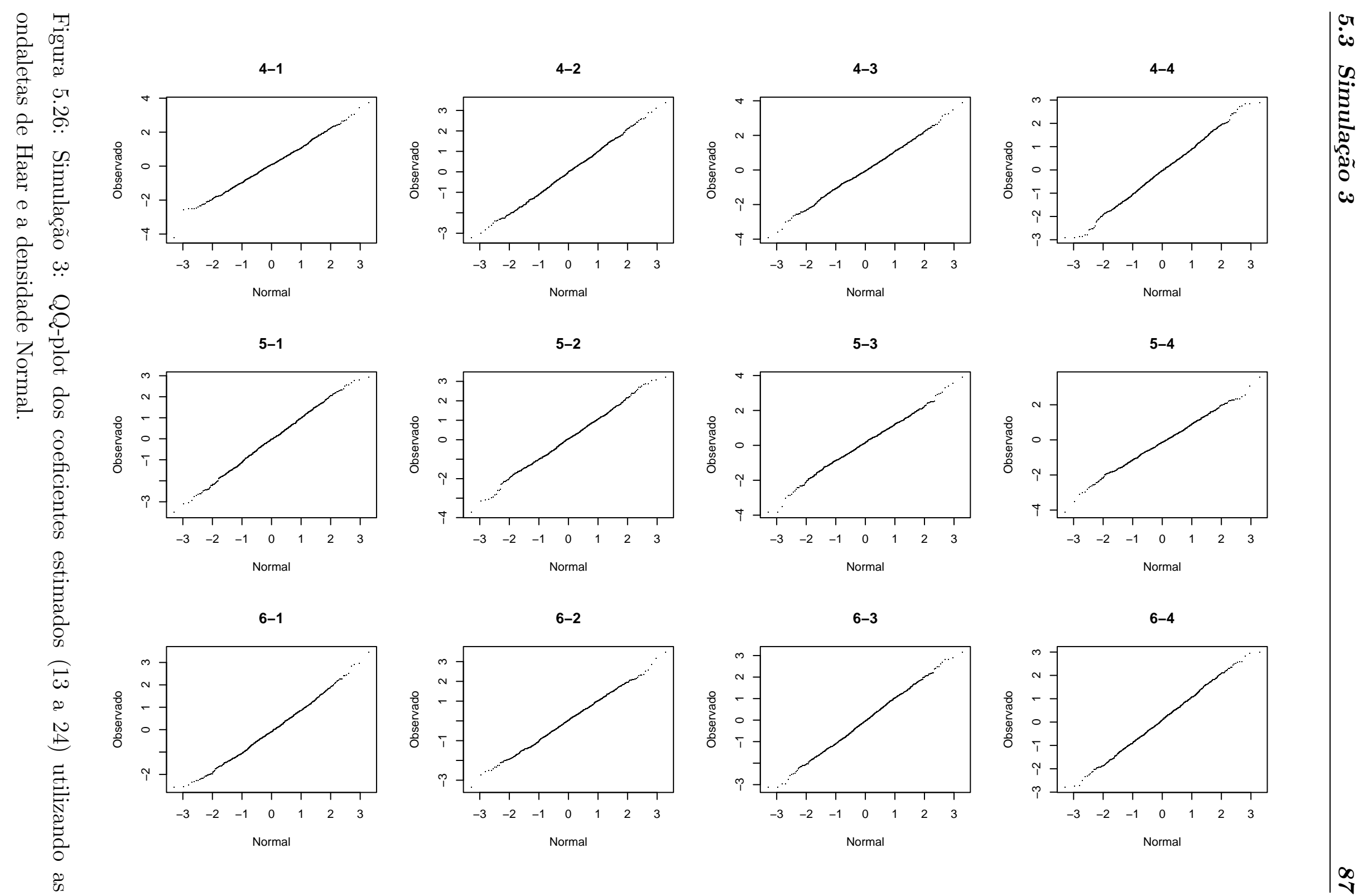

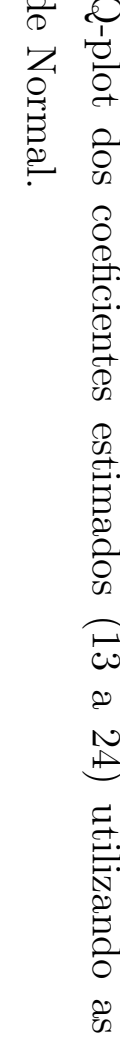
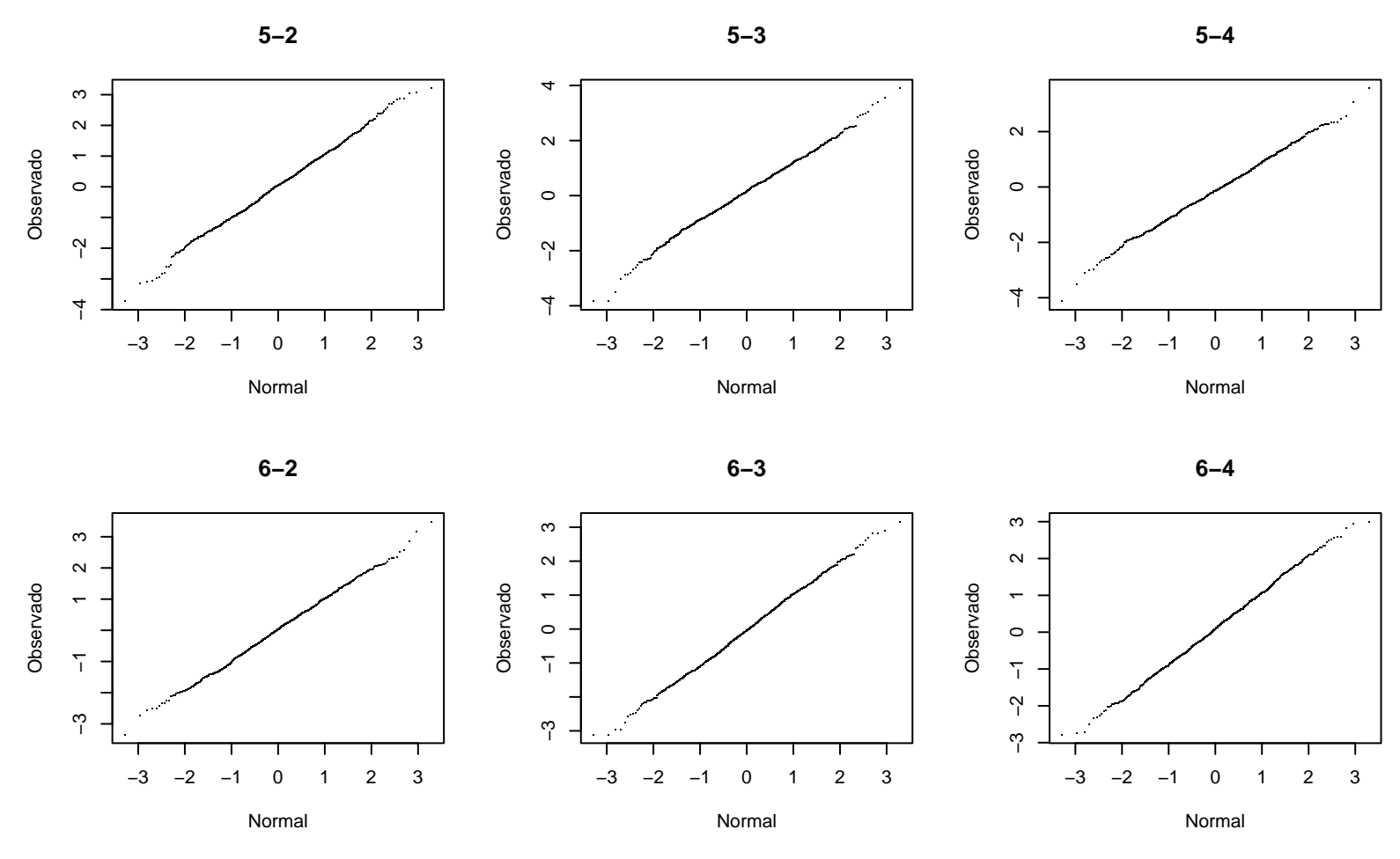


\subsection{Simulação 4}

Assim como no conjunto de simulações 2, desejamos avaliar o desempenho do teste de Wald para coeficientes variando no tempo, no entanto, nos casos em que a variação do coeficiente não é contínua. Em outras palavras, desejamos avaliar o poder do teste em situações em que a função de variação é uma função threshold. Dessa forma, consideramos um modelo semelhante ao modelo da sessão anterior, dado por

$$
x_{t}=u(t)+\mathbf{A}(t) x_{t-1}+\epsilon_{t},
$$

onde

$$
\mathbf{u}(t)=\left(\begin{array}{c}
0,25 f(t) \\
0,3
\end{array}\right), \mathbf{A}(t)=\left(\begin{array}{cc}
-0,3 g(t) & -0,4 g(t) \\
\theta f(t) & -0,3
\end{array}\right)
$$

$\mathrm{e}$

$$
\boldsymbol{\Sigma}(t)=\left(\begin{array}{cc}
0,5+0,5 f(t) & (0,5+0,5 f(t))(0,5+0,5 g(t)) \\
(0,5+0,5 f(t))(0,5+0,5 g(t)) & 2(0,5+0,5 g(t))
\end{array}\right) .
$$

Note que neste modelo, o coeficiente de causalidade das primeira série sobre a segunda, depende de um parâmetro $\theta$. O poder do teste de coeficientes variando no tempo é avaliado considerando-se os valores $0 ; 0,2 ; 0,4 ; 0,6 ; 0,8$ e 1,0 para $\theta$. Para verificarmos a influência do tamanho da amostra no teste, consideramos $T=64,128$ e 256. Foram utilizadas tanto as ondaletas de Haar quanto as ondaletas D8. Assim, realizaram-se 500 simulações para cada valor de $\theta$ e $T$, verificando-se a proporção de rejeição do teste. Os resultados deste conjunto de simulações são apresentados nas Tabelas 5.3 e 5.4, e nas Figuras 5.27 e 5.28.

\subsection{Simulação 5}

Em geral, as séries temporais de experimentos de ressonância magnética funcional (RMf) são de extensão pequena, tendo de 60 a 200 observações no tempo. No entanto, nosso maior interesse estão em experimentos com paradigmas periódicos, de forma que o indivíduo realiza um mesmo ciclo de tarefas diversas vezes durante uma única sessão. Assim, podemos utilizar a repetição dos ciclos como réplicas, aumentando a precisão das estimativas e aumentando o poder do teste. 


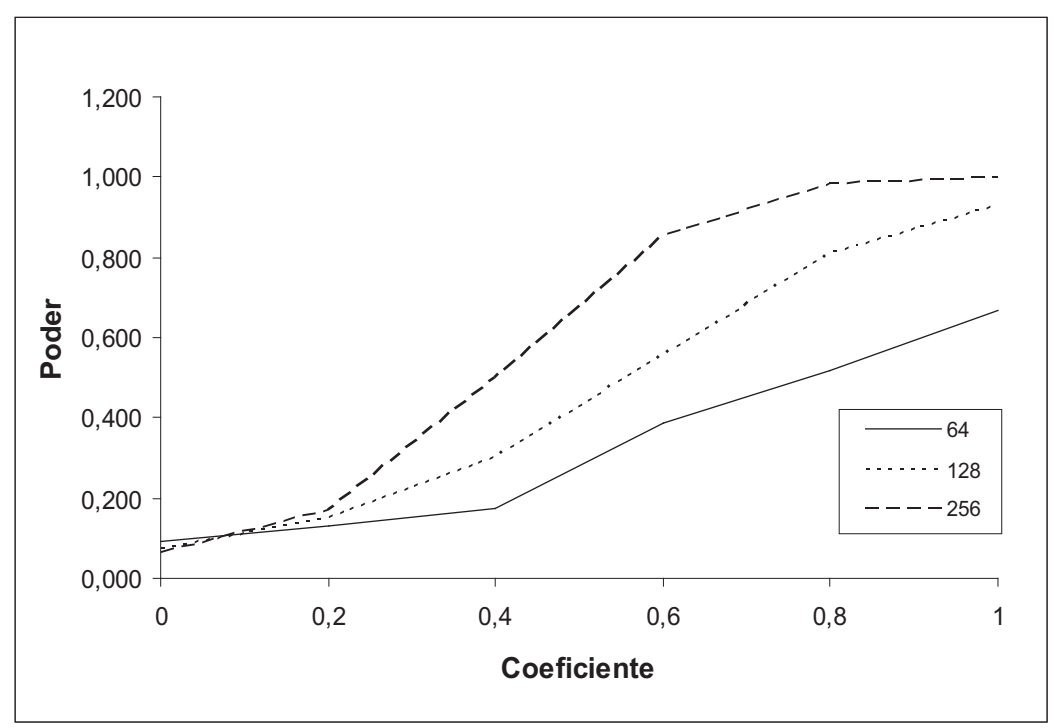

Figura 5.27: Poder do teste de coeficiente variando no tempo utilizando a ondaleta D8.

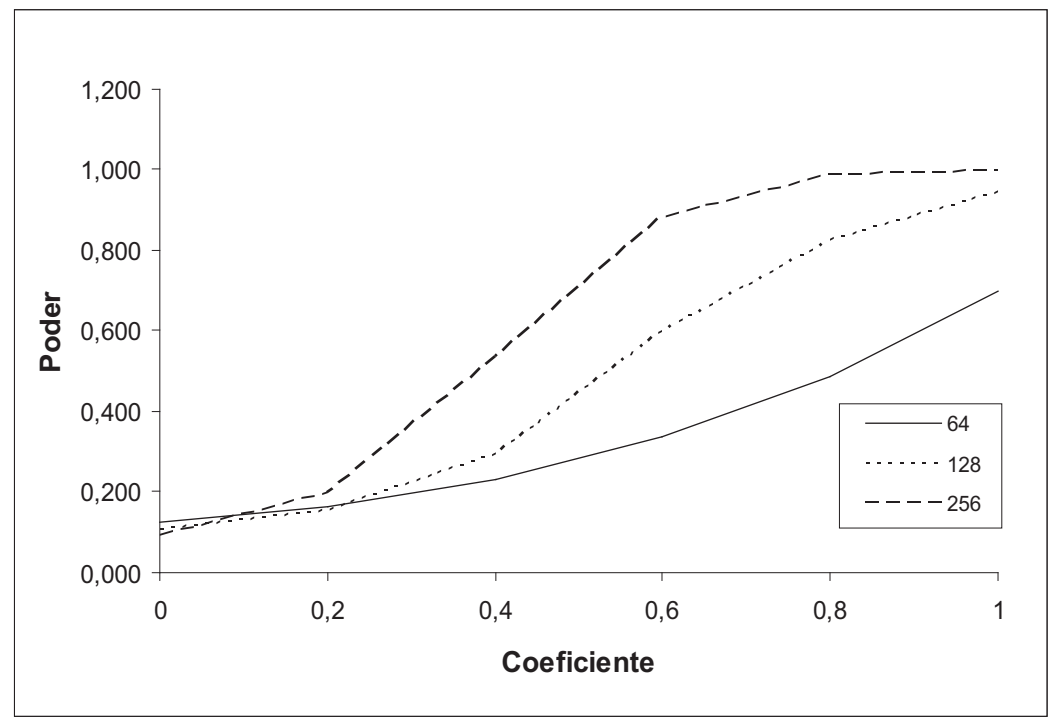

Figura 5.28: Poder do teste de coeficiente variando no tempo utilizando a ondaleta de Haar. 


\begin{tabular}{|c|c|cccccc|}
\hline Ondaleta & $\mathrm{T}$ & \multicolumn{7}{|c|}{$\theta$} \\
\hline \hline & & 0 & 0,2 & 0,4 & 0,6 & 0,8 & 1 \\
\hline \multirow{3}{*}{ D8 } & 64 & 0,094 & 0,130 & 0,176 & 0,386 & 0,518 & 0,670 \\
& 128 & 0,074 & 0,152 & 0,300 & 0,556 & 0,808 & 0,930 \\
& 256 & 0,062 & 0,168 & 0,500 & 0,854 & 0,982 & 0,998 \\
\hline \multirow{3}{*}{ Haar } & 64 & 0,126 & 0,162 & 0,232 & 0,336 & 0,486 & 0,698 \\
& 128 & 0,106 & 0,154 & 0,296 & 0,596 & 0,824 & 0,944 \\
& 256 & 0,090 & 0,196 & 0,536 & 0,880 & 0,990 & 0,996 \\
\hline
\end{tabular}

Tabela 5.2: Simulação 4: Proporção de Rejeição para o teste de funções de causalidade variando no tempo.

Considere uma série temporal 5-dimensional de um processo DVAR de ordem 1, com as especificações dos parâmetros $\mathbf{A}_{i}$, $\mathbf{u}$ e matriz de covariância $\mathbf{\Sigma}$ dados por:

$$
\begin{aligned}
& \mathbf{A}_{i}=\left(\begin{array}{ccccc}
0 & 0,2 & 0 & \cos \left(\frac{2 \pi t}{16}+\pi\right) / 2 & 0 \\
\operatorname{sen}\left(\frac{2 \pi t}{16}+\pi\right) / 4 & 0 & 0 & 0,3 & 0 \\
0 & 0 & 0,2 & 0 & \operatorname{sen}\left(\frac{2 \pi t}{16}+\pi\right) / 2 \\
0 & 0 & \cos \left(\frac{2 \pi t}{16}+\pi\right) / 4 & 0 & 0,3 \\
\operatorname{sen}\left(\frac{2 \pi t}{16}+\pi\right) / 4 & 0 & 0 & \cos \left(\frac{2 \pi t}{16}+\pi\right) / 4 & 0
\end{array}\right) \\
& \mathbf{u}=\left(\begin{array}{c}
\operatorname{sen}\left(\frac{2 \pi t}{16}+\pi\right) / 2 \\
\cos \left(\frac{2 \pi t}{16}+\pi\right) / 4 \\
0 \\
0
\end{array}\right) \\
& \boldsymbol{\Sigma}=\left(\begin{array}{ccccc}
0,49 & 0,147\left(1+\cos \left(\frac{2 \pi t}{16}\right) / 6\right) & 0 & 0 & 0 \\
0,147\left(1+\cos \left(\frac{2 \pi t}{16}\right) / 6\right) & 0,53\left(1+\cos \left(\frac{2 \pi t}{16}\right) / 6\right) & 0 & 0 & 0 \\
0 & 0 & 0,49 & 0 & 0 \\
0 & 0 & 0 & 0,49 & 0,147 \\
0 & 0 & 0 & 0,147 & 0,53
\end{array}\right) .
\end{aligned}
$$

Note que utilizando os parâmetros especificados anteriormente, temos uma estrutura periódica com ciclo de 16 observações. Dessa forma, considerando um total de $T=$ 96, temos seis réplicas deste ciclo. Assim, avaliou-se a performance do modelo DVAR utilizando-se 1000 simulações com a ondaleta D8, reescrevendo adequadamente a matriz 
modelo para réplicas. Os resultados são apresentados na Figura 5.29. As simulações mostram que a média das curvas estimadas são próximas das curvas teóricas. Além disso, não há muita variabilidade, indicando que o modelo DVAR apresenta uma boa performance considerando as réplicas, e enfatizando sua adequabilidade para dados de RMf.

\subsection{Conclusões}

Tendo em vista os resultados advindos de todos os conjuntos de simulações apresentados neste capítulo, concluímos:

- No geral, a metodologia proposta para a estimação de processos auto-regressivos multivariados com coeficientes variando no tempo apresenta uma performance satisfatória, com viés e variâncias pequenos;

- Os conjuntos de simulações 1 e 3 mostram que os estimadores possuem ligeiro viés, devido ao truncamento, mas as esperanças das funções estimadas são muito próximas às curvas teóricas;

- As curvas estimadas possuem baixa variabilidade, indicando uma boa precisão das estimativas;

- A utilização de bases não "adequadas", isto é, a utilização de ondaletas de Haar para a estimação de funções suaves, ou utilização da ondaleta D8 para estimação de funções escada, resulta em alto viés dos estimadores. No entanto, verifica-se que a longo prazo, as curvas estimadas acompanham bem as curvas teóricas;

- Independentemente do tipo de funções de base utilizada, os estimadores dos parâmetros do modelo parecem possuir distribuição assintótica gaussiana, como evidenciam os histogramas e qq-plots contruídos nos conjuntos de simulação 1 e 3;

- O estimador proposto para as funções variâncias e covariâncias dos erros do modelo apresenta uma performance satisfatória;

- O teste de Wald para coeficientes variando no tempo apresenta os resultados assintóticos esperados, mas a função poder apresenta um crescimento lento;

- Como esperado, quanto maior a extensão das séries temporais, melhor é a aproximação da distribuição da estatística e maior o poder do teste; 


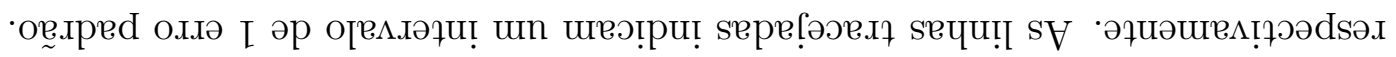

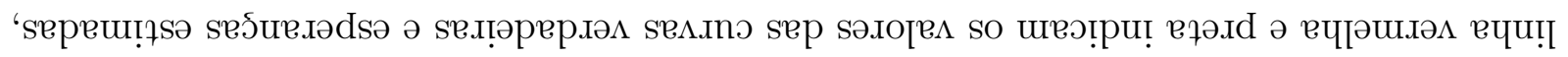

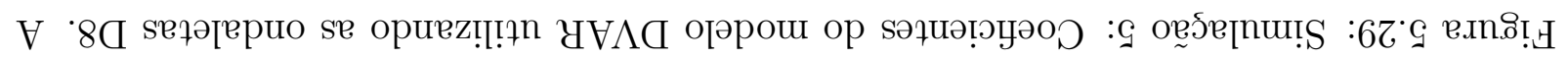
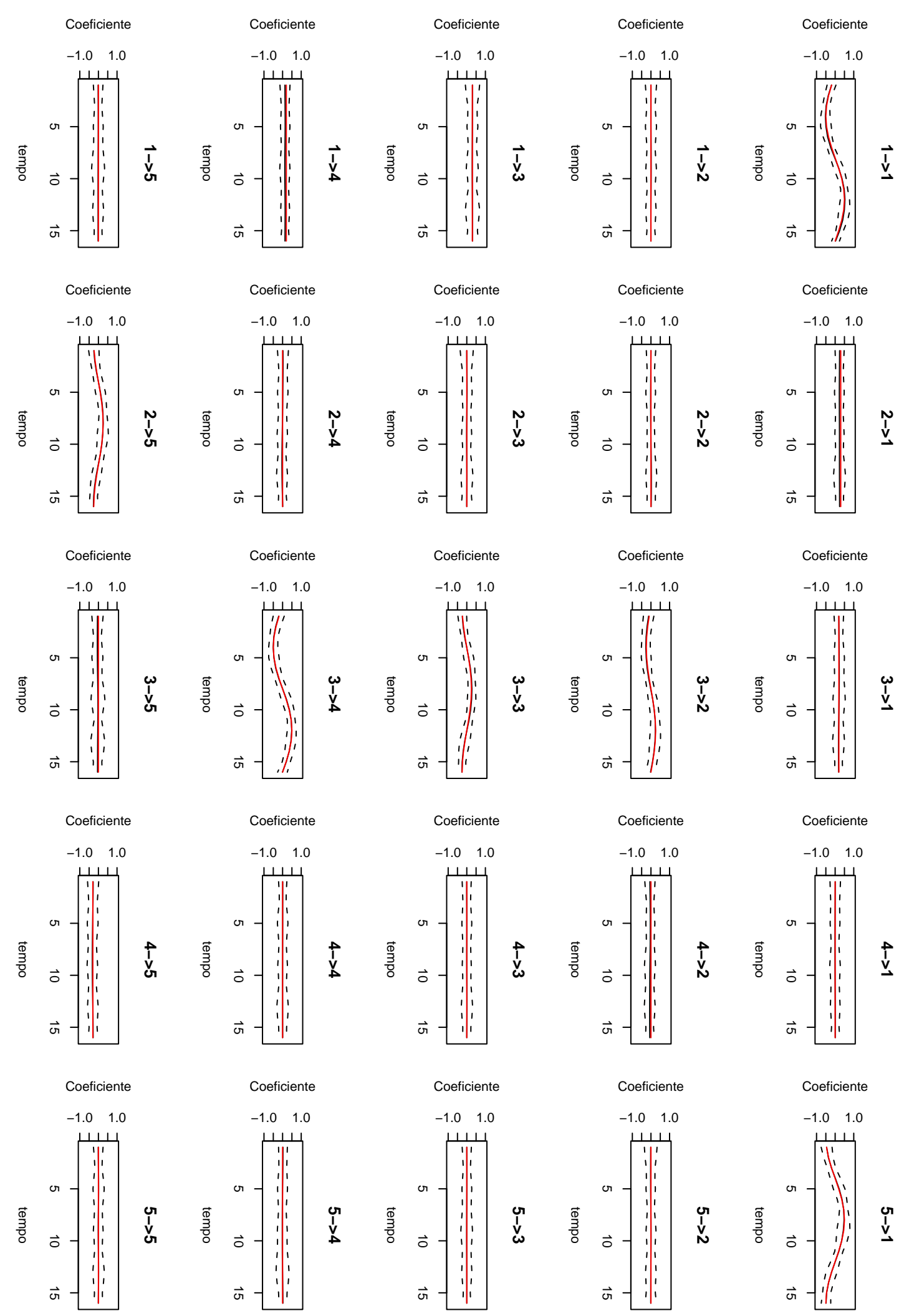
- A utilização de réplicas melhora significativamente a performance do estimador proposto, como evidenciam as Simulações 5. 


\section{Aplicações a Dados de RMf}

Neste capítulo apresentaremos algumas aplicações dos modelos multivariados dinâmicos sobre dados de ressonância magnética funcional. As análises de ativação cerebral e pré-processamento das imagens foram realizadas com a utilização do software XBAM (Brammer, 1997) e SPM (www.fil.ion.ucl.ac.uk/spm). O processo de estimação do modelo de conectividade foi implementado no software livre $\mathrm{R}$, com a utilização da biblioteca wavethresh e AnalyzeFMRI. A manipulação gráfica das imagens de ressonância magnética foi realizada no software MRICRO (www.mricro.org, desenvolvido por Chris Rorden).

\subsection{Experimento Motor}

Os dados deste experimento foram coletados no Instituto de Radiologia do Hospital das Clínicas de São Paulo - USP. Três indivíduos se voluntariaram para a participação deste estudo. O paradigma deste experimento é um desenho AB periódico simples, isto é, com a realização repetitiva e cíclica de duas atividades. Neste experimento, os indivíduos inicialmente permaneciam em repouso, apenas aguardando um sinal para entrarem na realização de atividade motora. Na atividade de movimento, os indivíduos foram instruídos a realizar uma atividade motora simples de fingertaping (Figura 6.1). Em seguida, os indivíduos retornavam ao estado de repouso inicial, aguardando novamente o sinal para recomeçarem a atividade. Este ciclo foi repetido 3 vezes, sendo que a duração de cada 
ciclo era de 60 segundos (20 observações temporais).

As imagens foram coletadas em um sistema GE 1,5 Tesla Signa equipado com um gra-

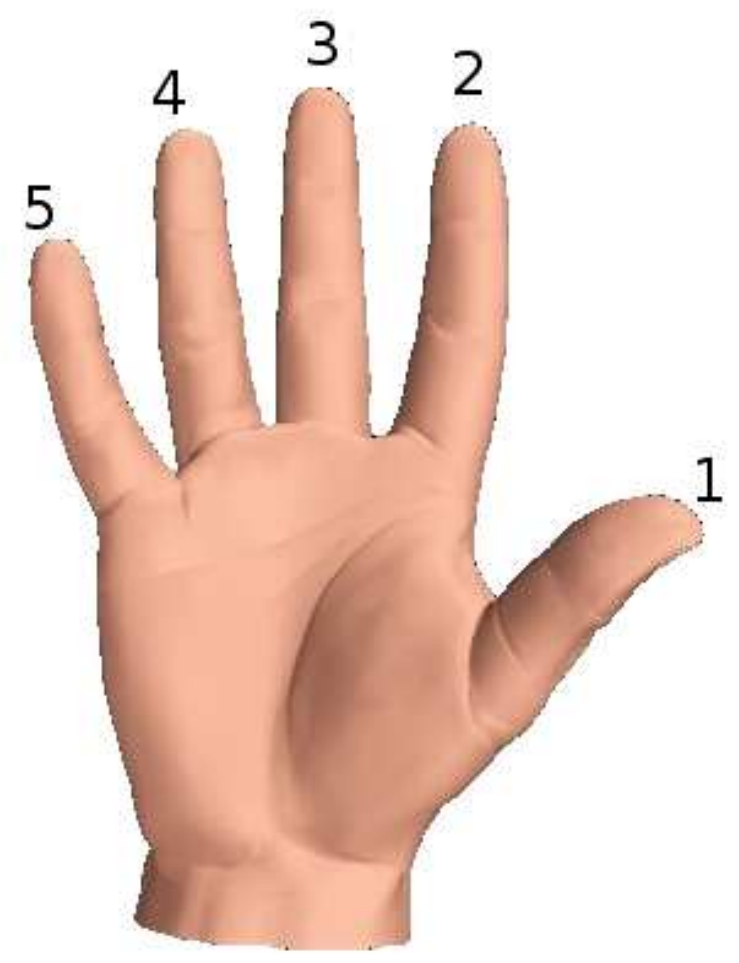

Figura 6.1: Fingertaping: os indivíduos deveriam realizar movimentos com o dedo, tocado ciclicamente os dedos 2,3,4 e 5 no dedo 1 .

diente $23 \mathrm{mT} / \mathrm{m}\left(\mathrm{TE}=40 \mathrm{~ms}, \mathrm{TR}=3000 \mathrm{~ms}, \mathrm{FA}=75^{\circ}, \mathrm{FOV}=240 \mathrm{~mm}\right.$, resolução de $64 \times 64$ voxels, 8 slices, espessura de $7,0 \mathrm{~mm}$, gap $=0.7 \mathrm{~mm}$ ) orientado no plano AC-PC. Foram coletados 60 volumes, e portanto, a extensão da série temporal para cada voxel é de tamanho 60. As imagens foram corrigidas por movimento do indivíduo, onde todas as imagens foram realinhadas em relação ao primeiro volume. As imagens também foram corrigidas por histórico de intensidade de spins e por tempo de aquisição dos slices. Além disso, também foi aplicado um filtro espacial gaussiano de $5 \mathrm{~mm}$. Mapas de ativação para os três indivíduos foram construídos (utilizando o software XBAM) e são apresentados na Figura 6.2. 
Verifica-se que para todos os indivíduos, houve forte ativação na área motora primária.

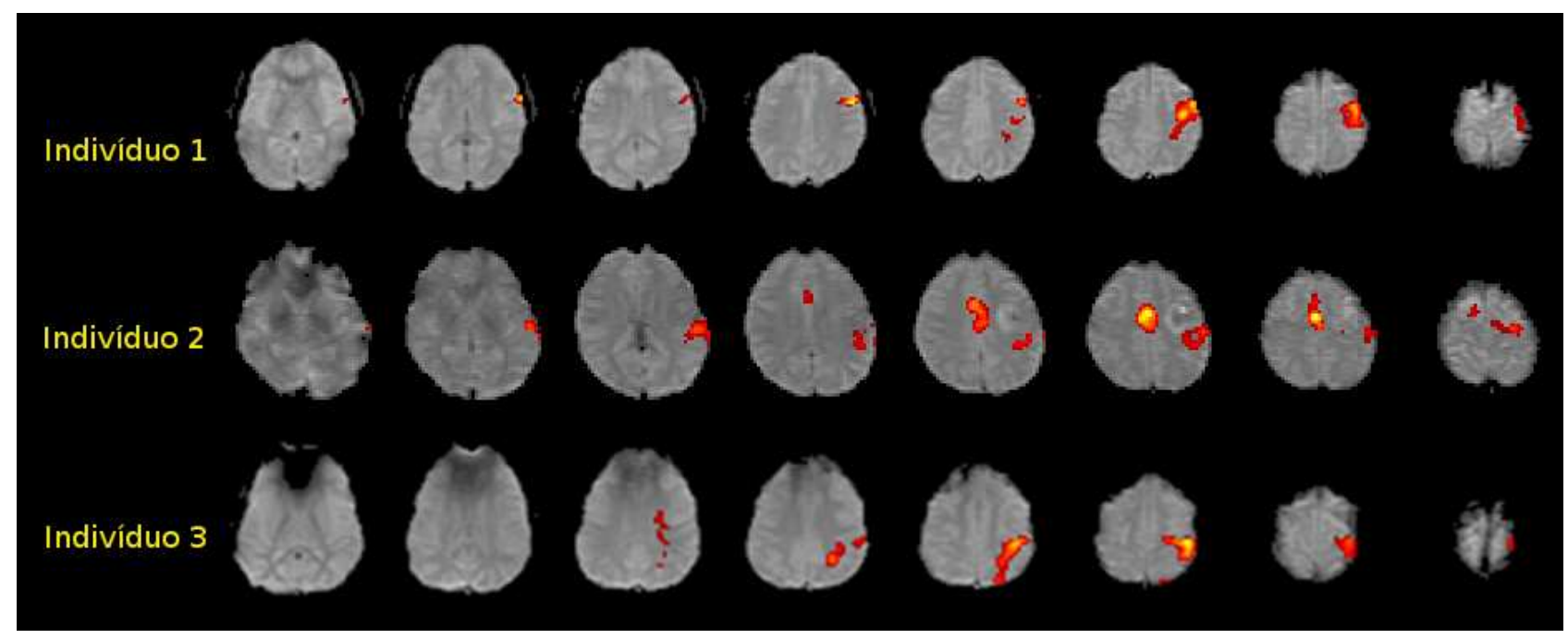

Figura 6.2: Mapa de ativação dos três indivíduos para a atividade de fingertaping.

Para cada indivíduo, a série temporal do voxel com maior ativação (local maxima = voxel com menor p-valor) é mostrada na Figura 6.3.

Considere agora, o interesse em avaliar quais áreas do cérebro enviam informações para a área motora primária, tanto no estado de descanso quanto na atividade de movimentação dos dedos. Além disso, deseja-se avaliar como a alteração dessas áreas se dá ao longo no tempo. Dessa forma, utilizou-se a modelagem de um DVAR proposto neste trabalho, assumindo-se que para cada ciclo do experimento, a estrutura da alteração de conectividade se dá da mesma forma, ou ao menos de forma semelhante. Em outras palavras, consideramos cada ciclo, como réplicas de uma atividade de repouso seguida de atividade motora. Assim sendo, representando a série temporal da área motora primária, utilizou-se a média dos $5 \times 5$ voxels, centrados no local maxima da área motora. Assim, para todo o resto do cérebro, aplicou-se a modelagem DVAR bivariadamente, utilizando a série representativa da área motora e a série de cada outro voxel do volume. No entanto, para uma melhor utilização das funções de ondaletas (a extensão da série deve ser uma potência de 2), cada ciclo composto de 20 volumes foi reamostrado para 16 volumes, utilizando interpolações via splines cúbicas. Utilizou-se as ondaletas D8 periódicas. Para cada instante de tempo, as áreas que enviam informações para a área motora primária são apresentadas nas Figuras 6.4, 6.5 e 6.6.

Considerando os mapas de conectividade das áreas que enviam informações para 

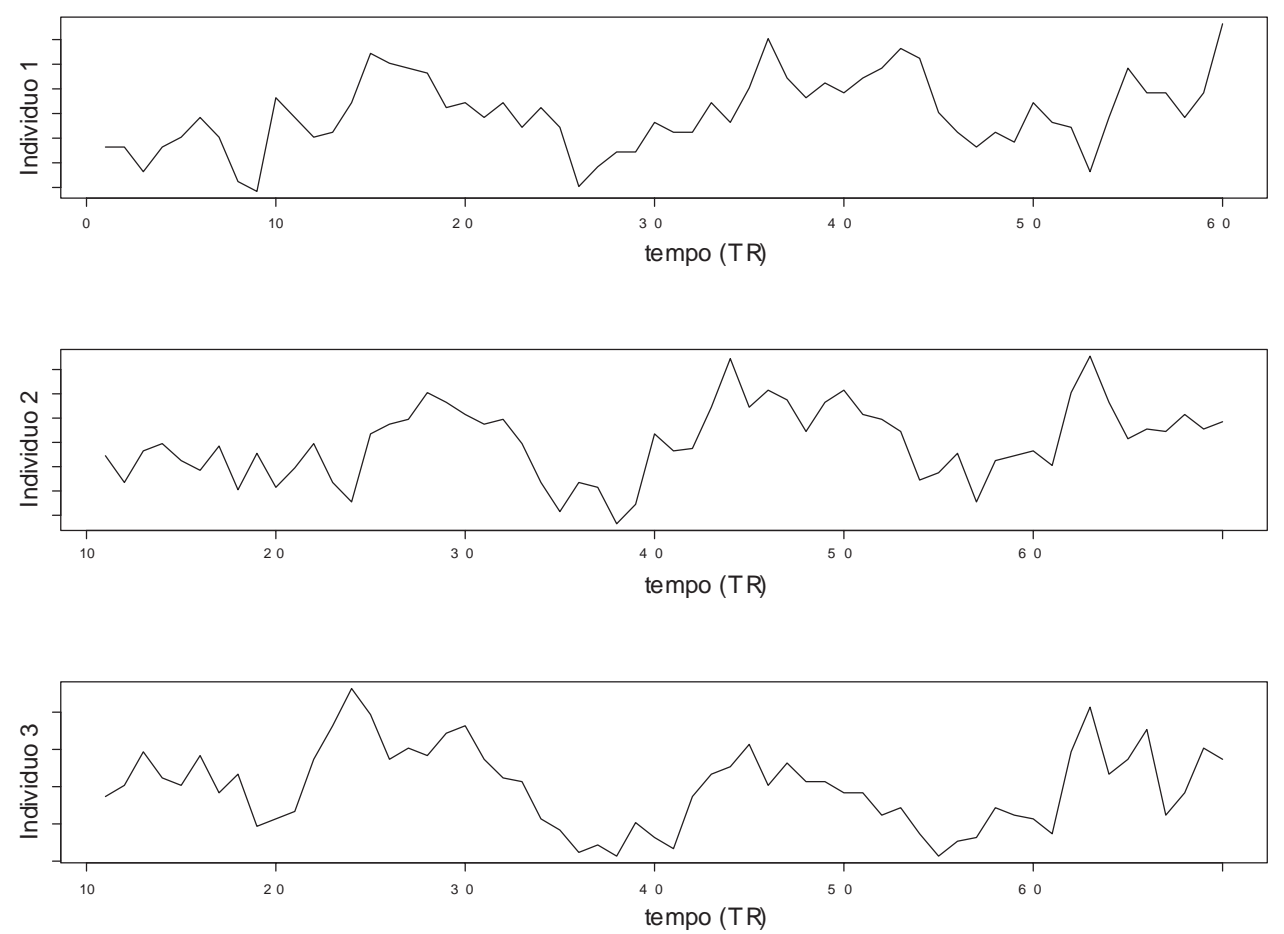

Figura 6.3: Séries Temporais do Local Máxima da área motora de cada indivíduo.

a área motora dos três indivíduos, observa-se um padrão da alteração de conectividade ao longo do tempo. Inicialmente, verifica-se que as área que enviam informações para a área motora primária situam-se na região pré-frontal, envolvendo também áreas parietais e áreas pré-motoras. Em seguida, após o início do estado de descanso, verifica-se que há uma migração das áreas que enviam informação para regiões posteriores. A partir do momento em que a atividade motora é iniciada, as áreas mais influentes voltam a ser as regiões anteriores. Estudos prévios (Lee, Chang et al. (1999), Ohara, Mima et al. (2001), Cunnington, Windischberger et al. (2002)) evidenciam que a região pré-frontal desempenha um papel importante na preparação de atividades motoras. Além disso, a detecção de áreas suplementares e pré motoras como fontes de informações para a área motora primária é razoável, pois outros estudos (Cui, Li et al. (2000), D’Esposito, Ballard et al. (2000), Toni, Thoenissen et al. (2001)) também mostraram que estas áreas estão relacionadas com a preparação do movimento. Sabe-se que estas áreas estão constantemente enviando informações para área motora primária, entretanto verifica-se através da modelagem DVAR que esta conectividade é pronunciada durante a realização da atividade motora. Um outro fato importante, é que o envolvimento de regiões parietais está possivelmente relacionado com a monitoração do movimento (Coull, Frith et al.,2000; Hall, 


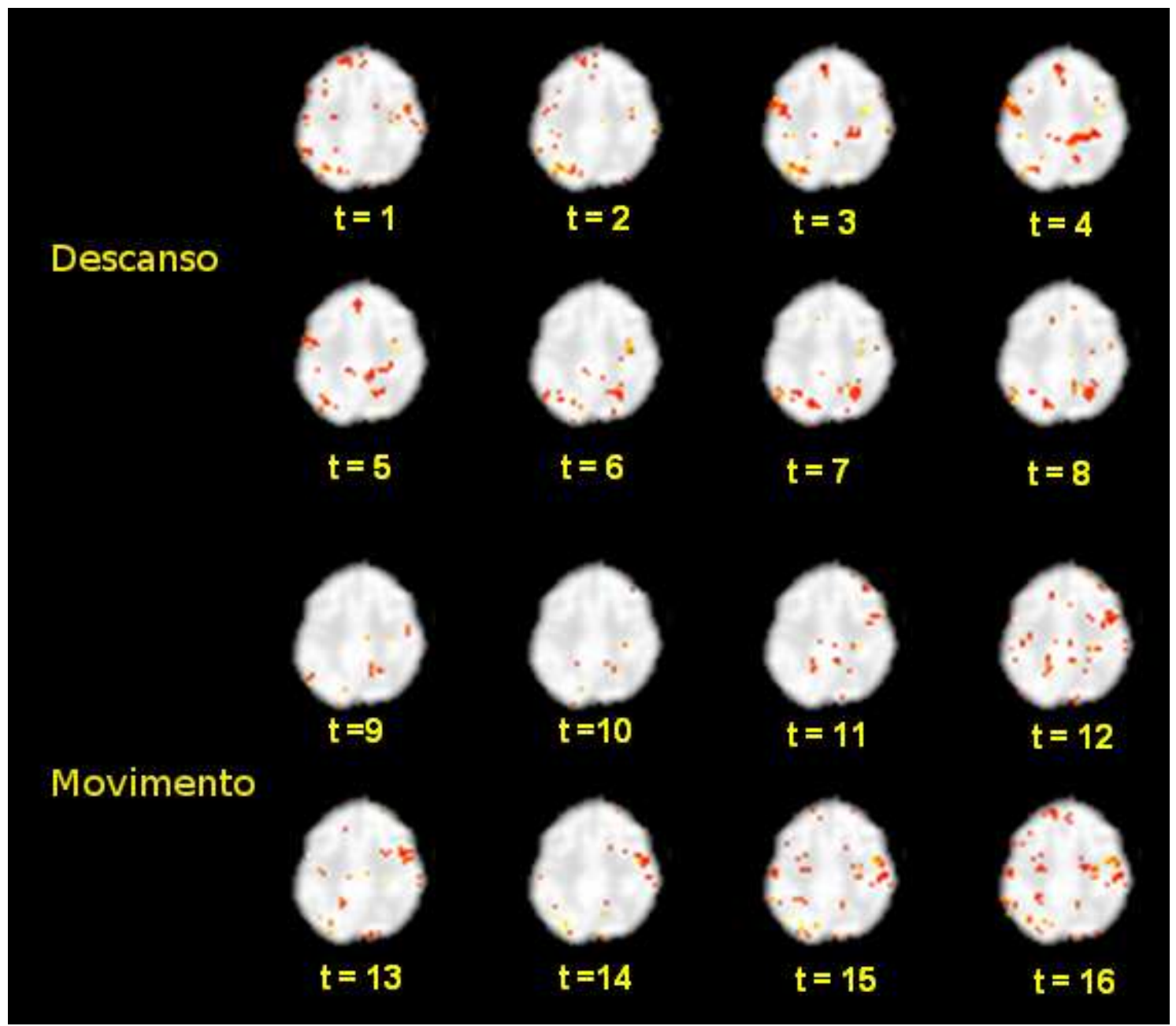

Figura 6.4: Indivíduo 1: Áreas que enviam informações para a área motora primária. 


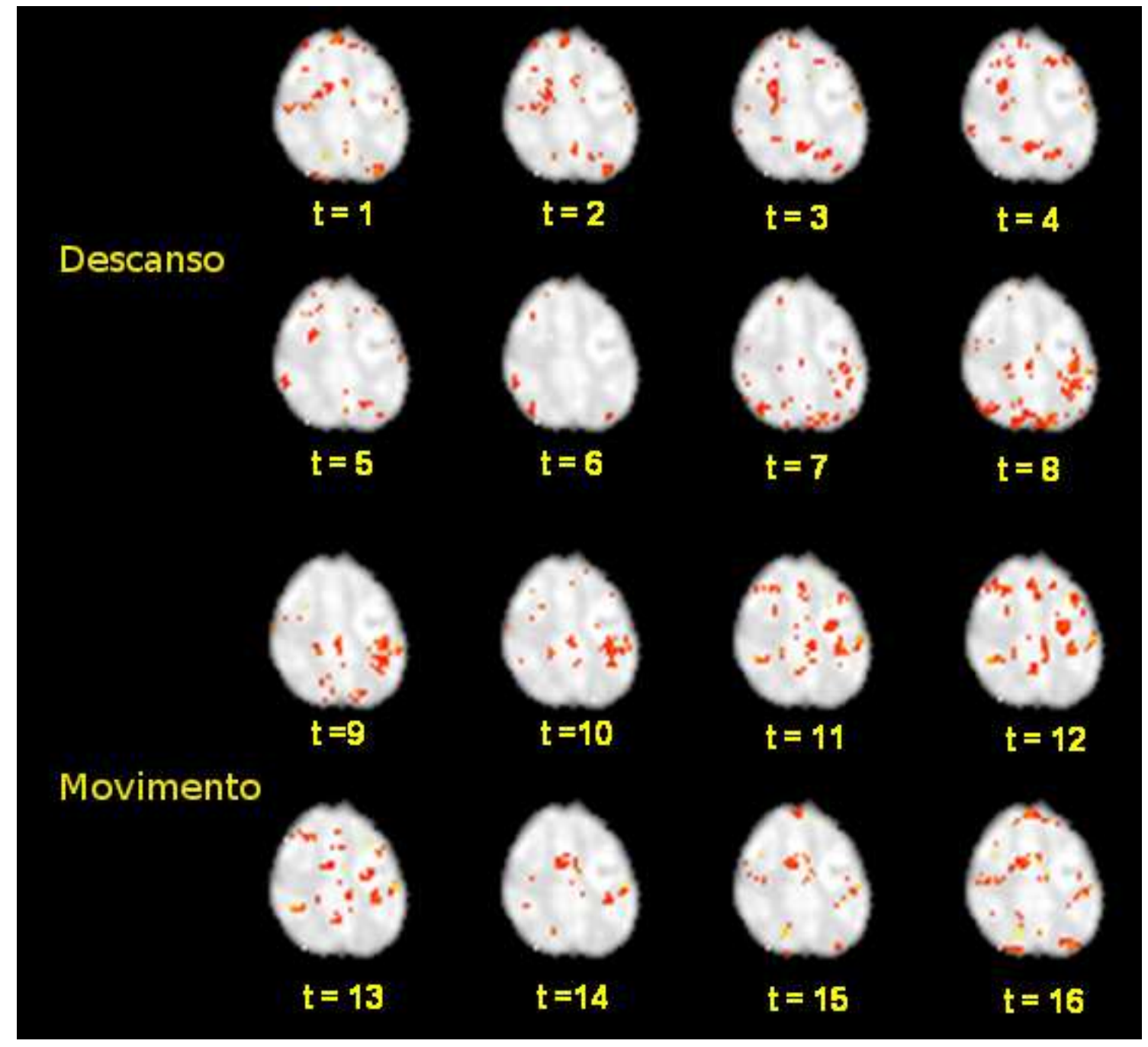

Figura 6.5: Indivíduo 2: Áreas que enviam informações para a área motora primária. 


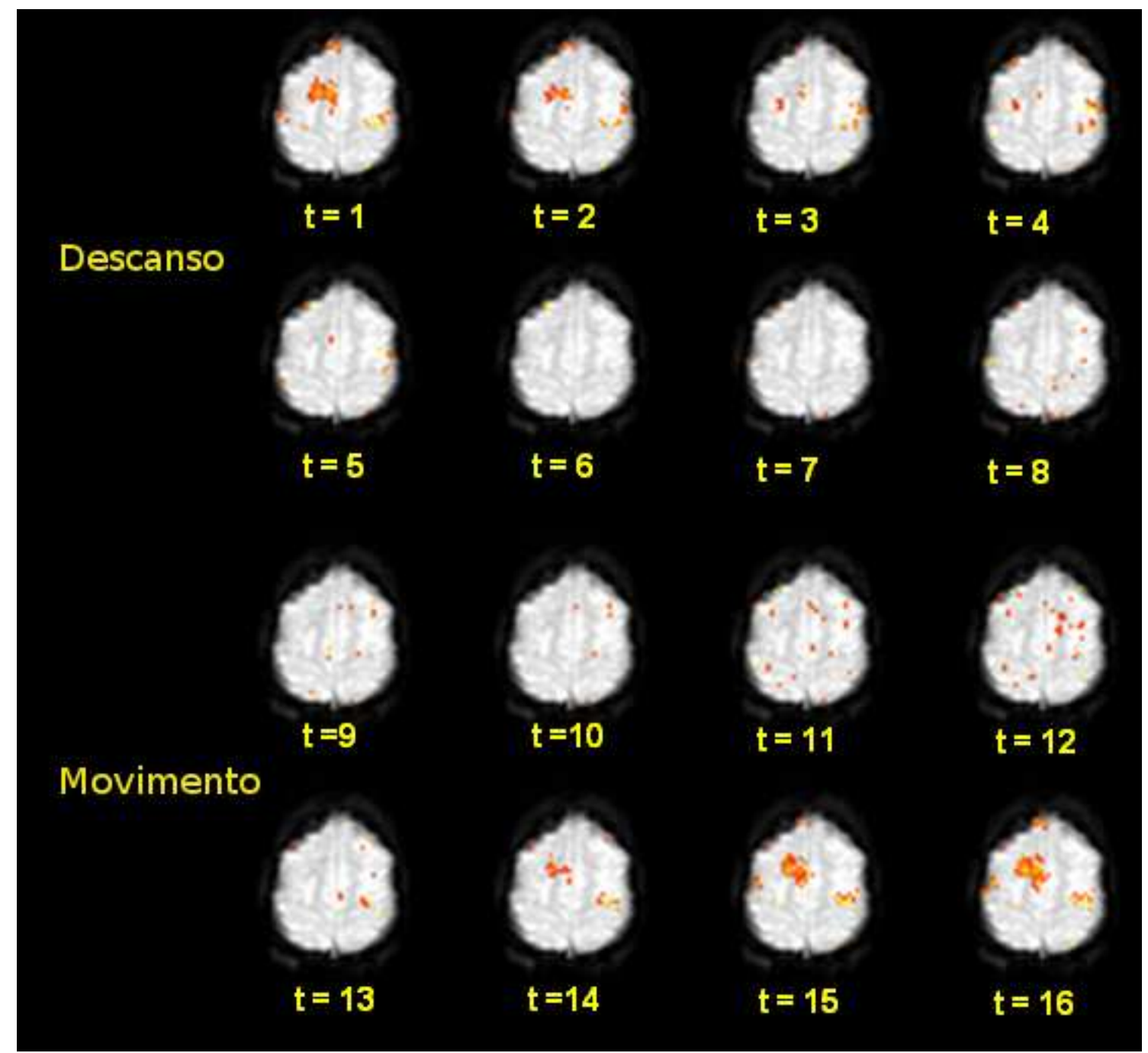

Figura 6.6: Indivíduo 3: Áreas que enviam informações para a área motora primária. 
Haggard et al.,2000; Lutz, Specht et al.,2000).

O modelo DVAR também pode ser aplicado à séries temporais multivariadas (i.e., com dimensão maior que dois), de forma a obter um melhor entendimento a respeito do relacionamento das áreas cerebrais envolvidas. Dessa forma, considerando os mapas de conectividade variando no tempo previamente calculados, selecionamos cinco regiões de interesse:

1) Local máxima da área motora primária esquerda (LM1) no giro pré-central;

2) Giro do cingulo anterior ( $\mathrm{ACg})$

3) Área pré-motora (SMA);

4) Área pré-motora direita (RpM1), localizado na região direita do giro pré-central;

5) Precuneus anterior $(\mathrm{ApC})$.

As áreas selecionadas já foram previamente descritas e relacionadas ao controle de movimento (Kermadi, Liu et al.,(2000); Wenderoth, Debaere et al.,(2005a)) e também apresentam participação importante no aprendizado motor (Jancke, Himmelbach et al.,(2000); Kurata, Tsuji et al.,(2000)). O modelo DVAR para os sinais BOLD destas áreas foi estimado e utilizando o teste de Wald para contrastes, verificamos quais conexões são estatisticamente significativas. A hipótese nula para este teste é que todos os coeficientes das expansões de ondaletas para uma determinada função de conectividade são iguais à zero. Em outras palavras, verificou-se estatisticamente se as funções de conectividade entre duas áreas cerebrais era uma função nula e constante ao longo do tempo. As séries temporais do BOLD das áreas selecionadas são apresentadas na Figura 6.7. A localização cerebral das áreas selecionadas e também o diagrama de conectividades estatisticamente significativas (poder do teste $\alpha=0.05$ ) são apresentados na Figura 6.8.

Analisando a evolução das funções de conectividade ao longo do tempo, verifica-se que há fluxo de informação com origem nas áreas ApC e SMA para a área LM1. No entanto, durante a atividade motora, a conectividade "positiva" de ApC para SMA torna-se "negativa", e o fluxo de SMA para LM1 tem intensidade reduzida. Além disso, verifica-se que o fluxo de informação da área LM1 para RpM1 é drasticamente elevada durante o movimento. A área $\mathrm{ACg}$ apresenta um efeito positivo durante o descanso, mas esta situação reverte-se durante o movimento, de forma que este efeito torna-se negativo.

Além disso, o padrão de conectividade ilustrado pela análise é interessante, pois a evolução temporal descrita no diagrama de conectividade é coerente com estudos prévios realizado em humanos e animais (Stephan, Binkofski et al.,(1999); Kermadi, Liu et 

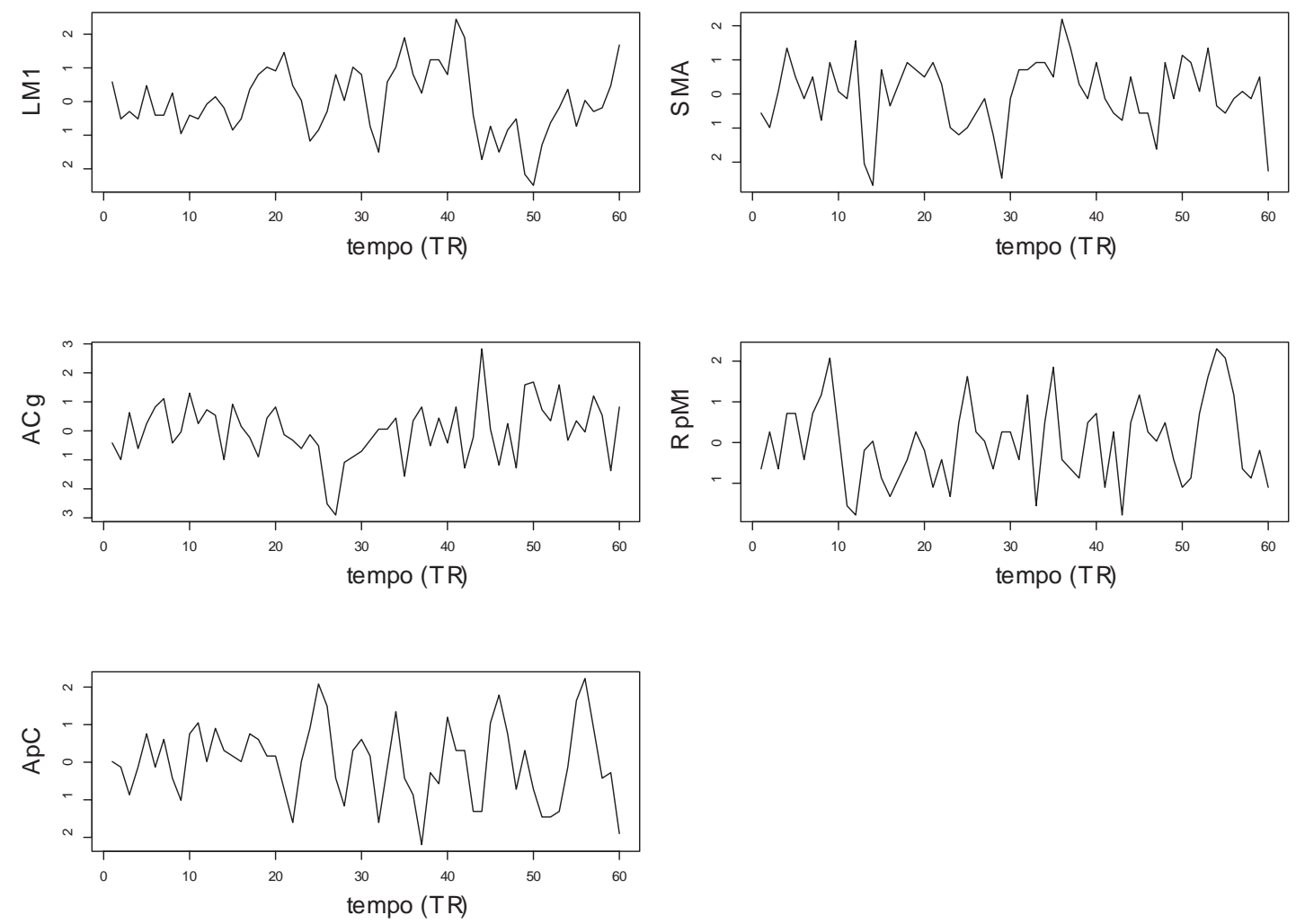

Figura 6.7: Séries temporais das cinco regiões de interesse. 

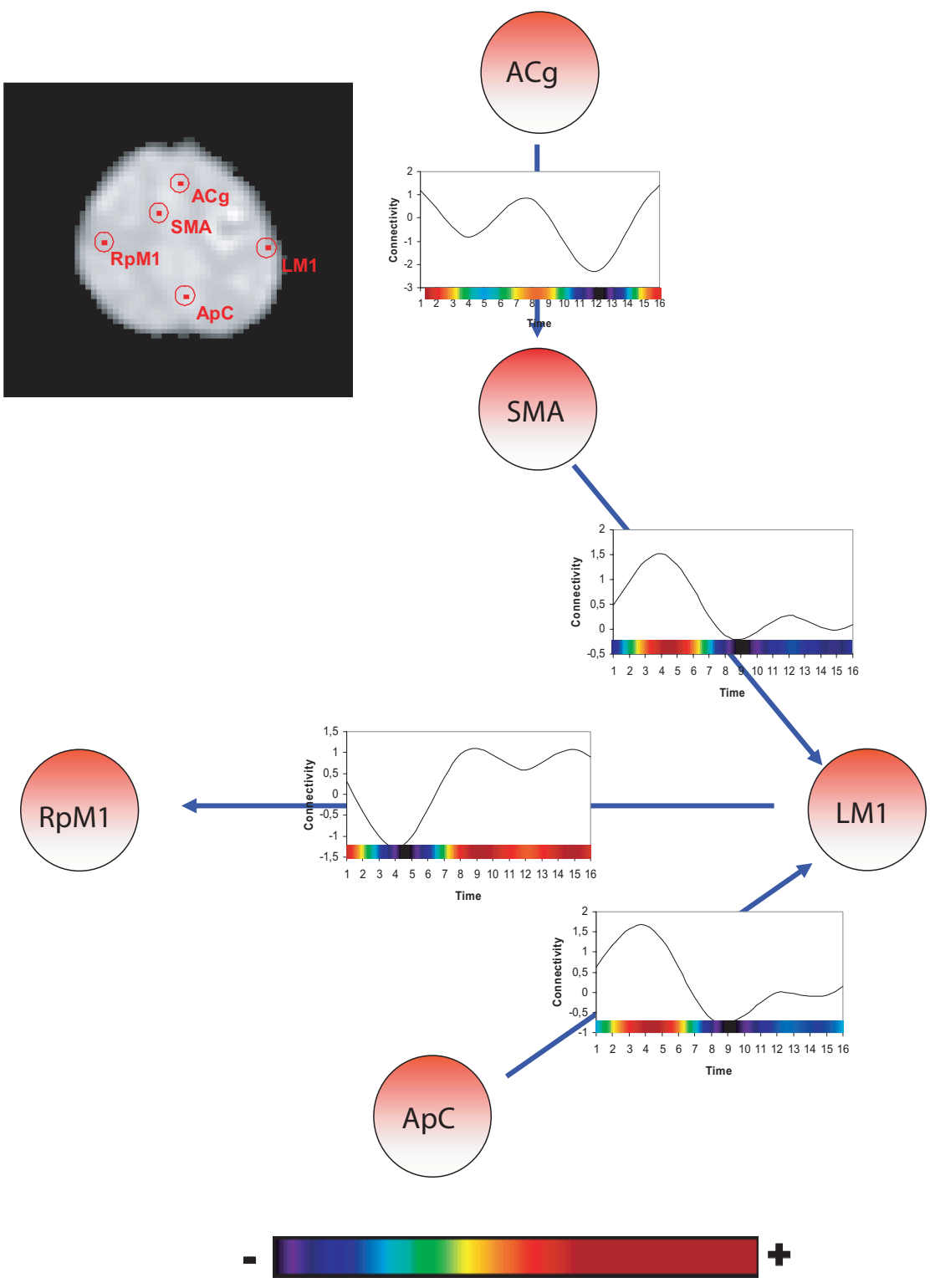

Figura 6.8: Diagrama de conectividade para o experimento motor. 
al.,(2000)). A região ACg é descrita como mediadora de processos que envolvem integração e controle bimanual. Da mesma forma, a região suplementar motora está presente na execução de tarefas que envolvem complexidade e freqüência rítmica no movimento das mãos (Wenderoth, Debaere et al.,(2004); Wenderoth, Debaere et al.,(2004); Wenderoth, Debaere et al.,(2005a)). Além disso, acredita-se que o precuneus dorso lateral anterior esteja relacionado aos aspectos atencionais de atividades motoras (Wenderoth, Debaere et al.,2005b). Na análise descrita, a função de conectividade estimada entre esta área e a área motora primária sugere que esta influência é maior durante a atividade de descanso, possivelmente indicando uma expectativa.

O fluxo de informações da área ACg para SMA também é interessante, pois a participação destas áreas durante o planejamento do movimento é descrito por Viallet (Viallet, Massion et al., 1992). A área SMA pode ser dividida em outras pequenas áreas, de forma que a literatura indica que área pré-SMA é mais ativa durante o periodo de preparação do movimento, do que durante a execução do movimento em si (Matteli, Luppino et al.,1991); Luppino, Matelli et al.(1993); Rizzolatti, Luppino et al.(1996)). Na modelagem do DVAR, verifica-se que a função de conectividade entre essas áreas apresenta um ligeiro pico durante a transição das duas condições, sendo coerente com a idéia da participação do ACg e SMA na seleção de ações (Rushworth, Walton et al.,2004)

\subsection{Doença de Parkinson}

A Doença de Parkinson é uma doença degenerativa com prevalência de aproximadamente um caso para cada mil pessoas com idade superior à 50 anos (Jack Jr. et al., 2002). A doença é freqüentemente caracterizada por distúrbios nos sistemas motores e cognitivos, e até o presente momento, ainda não foram descobertos procedimentos para a cura definitiva. Os principais sintomas deste mal são tremores em repouso, movimentos em roda denteada, bradicinesia, resistência à movimentação passiva, depressão, perda de memória, ansiedade, lentidão de pensamento, dentre outros.

Os mecanismos da doença são ainda pouco conhecidos. No entanto, existem algumas conjecturas de que a doença é decorrente de uma redução de ativação em áreas importantes do sistema motor, como a área motora primária (M1), a área pré-motora e 
área suplementar motora devido a alterações na estrutura de conectividade do tálamo e núcleos subtalâmicos (Nasser et al., 2002). Através de estudos utilizando primatas, DeLong (1990) também obteve evidências de alterações na conectividade do circuito motor, principalmente na intensidade do fluxo de informações baso-tálamo-corticais.

Assim sendo, verifica-se que o estudo da conectividade do sistema motor é de fundamental importância para uma melhor caracterização da doença. Portanto, há uma forte motivação da aplicação do modelo DVAR, para experimentos de ressonância magnética funcional envolvendo indivíduos com o mal de Parkinson.

Os experimentos em RMf foram aprovados pela Comissão de Ética para Análise de Projetos de Pesquisa - CAPPesq/HC - FMUSP e da Comissão de Ensino e Pesquisa do Departamento de Radiologia (831/00). Todos os voluntários assinaram um termo de consentimento de participação no estudo, não havendo nenhuma gratificação financeira. Quatorze indivíduos, sendo sete normais e sete pacientes, foi o total de voluntários neste estudo. O experimento de ressonância magnética funcional envolveu um paradigma motor (fingertap com a mão direita) com desenho relacionado evento (event-related design). Cada voluntário permanecia em repouso no scanner, e a cada vinte segundos recebia um estímulo auditivo, indicando que uma e apenas uma seqüência de fingertap deveria ser executada. A duração do experimento foi de 200 segundos, totalizando a realização de 10 ciclos. A realização da atividade motora tinha início no primeira observação de cada ciclo.

O conjunto de dados foi coletado no Departamento de Radiologia dos Hospital das Clínicas - Universidade de São Paulo em um scanner Signa LX 1.5T (GE, Milwaukee, USA) equipado com um gradientes $23 \mathrm{mT} / \mathrm{m}$ e capabilidade ecoplanar. As imagens coletadas são baseadas em gradientes 2D eco EPI com um $T R=2000 \mathrm{~ms}$, $T E=40 \mathrm{~ms}$, $F A=90, b w=64 k H z, F O V=20 \mathrm{~cm}, 64 \times 64$ pixels, slices com $7 \mathrm{~mm}$ de espessura, com gap de $0.7 \mathrm{~mm}$ entre os 15 slices adquiridos, orientados paralelamente ao plano bicomissural. Para cada indivíduo, foram adquiridos 100 volumes, resultando em séries temporais multivariadas com 100 observações, em um exprimento com um ciclo de 10 observações.

A imagens foram pré-processadas considerando correções de movimento, suavização espacial, realinhamento temporal e normalização para o espaço estereotático de Talairach e Tournoux (1988). O pré-processamento e análise de mapas de ativação foram realizados utilizando o software SPM2 (http : //fil.ion.ucl.ac.uk/spm). Os mapas de ativação dos grupos normais e Parkinson são apresentados nas iguras 6.9 e 6.10 respectivamente. 
Para a aplicação do modelo DVAR, considerou-se os mapas de ativação do grupo normal e selecionou-se 3 regiões de interesse (ROIs) representadas pelo sinal BOLD das áreas com menor p-valor de ativação (local maxima). As áreas de interesse são cerebelo direito (CER), área suplementar motora (SMA) e área motora primária esquerda (M1) (vide Figura 6.11). Dessa forma, considerando-se as 3 ROIs, o modelo DVAR foi ajustado para cada indivíduo separadamente, assumindo cada ciclo do experimento como uma réplica. Assim, a função de conectividade de um ciclo foi estimada, e tem a extensão de 10 medidas no tempo.

De forma a comparar as funções de conectividade entre as ROIs para os dois grupos, realizou-se um teste não-paramétrico de Mann Whitney para cada instante de tempo (nível de significância de 0.05). As curvas de conectividade média dos dois grupos e o resultado do teste para diferenças das curvas são apresentados na Figura 6.12.

Através da modelagem da estrutura de conectividade utilizando o modelo com coeficientes variando no tempo, verifica-se que durante e alguns segundos após a execução da tarefa motora, há diferenças estatisticamente significantes na intensidade do fluxo de informações de M1 para SMA. Além disso, não há evidências para afirmar que há diferença na conectividade de CER para SMA e de M1 para CER.

O cerebelo está envolvido tanto na movimentação ativa e passiva integrando o córtex pré-frontal dorsolateral (intenção) e a medula (execução). Além disso, o cerebelo também participa no armazenamento da programação/planejamento do movimento, em uma rede neuronal que envolve a área suplementar motora e áreas pré-motoras (Ito et al., 1999). Algumas teorias sugerem que os pacientes do mal de Parkinson possuem alterações na programação motora, resultante de uma perturbação na conectividade das redes tálamocorticais (DeLong et al., 1990). Assim sendo, é razoável esperar alterações no fluxo de informações entre áreas motoras primárias e áreas pré-motoras, dado que ambos participam na programação e execução do movimento.

Uma conjectura sugerida pela análise de conectividade é a de que o nível basal do fluxo de informações de SMA para CER e de M1 para CER é alterado nos pacientes que sofrem do mal Parkinson, e tal fato pode estar possivelmente relacionado aos tremores involuntários durante o repouso (movimentação passiva). No entanto, verifica-se que essa estrutura de conectividade aproxima-se da estrutura do grupo saudável após a execução de uma tarefa motora envolvendo ativação cortical. Além disso, alterações no fluxo de informações de M1 para SMA logo após a execução da tarefa é esperada, dado que ambas 


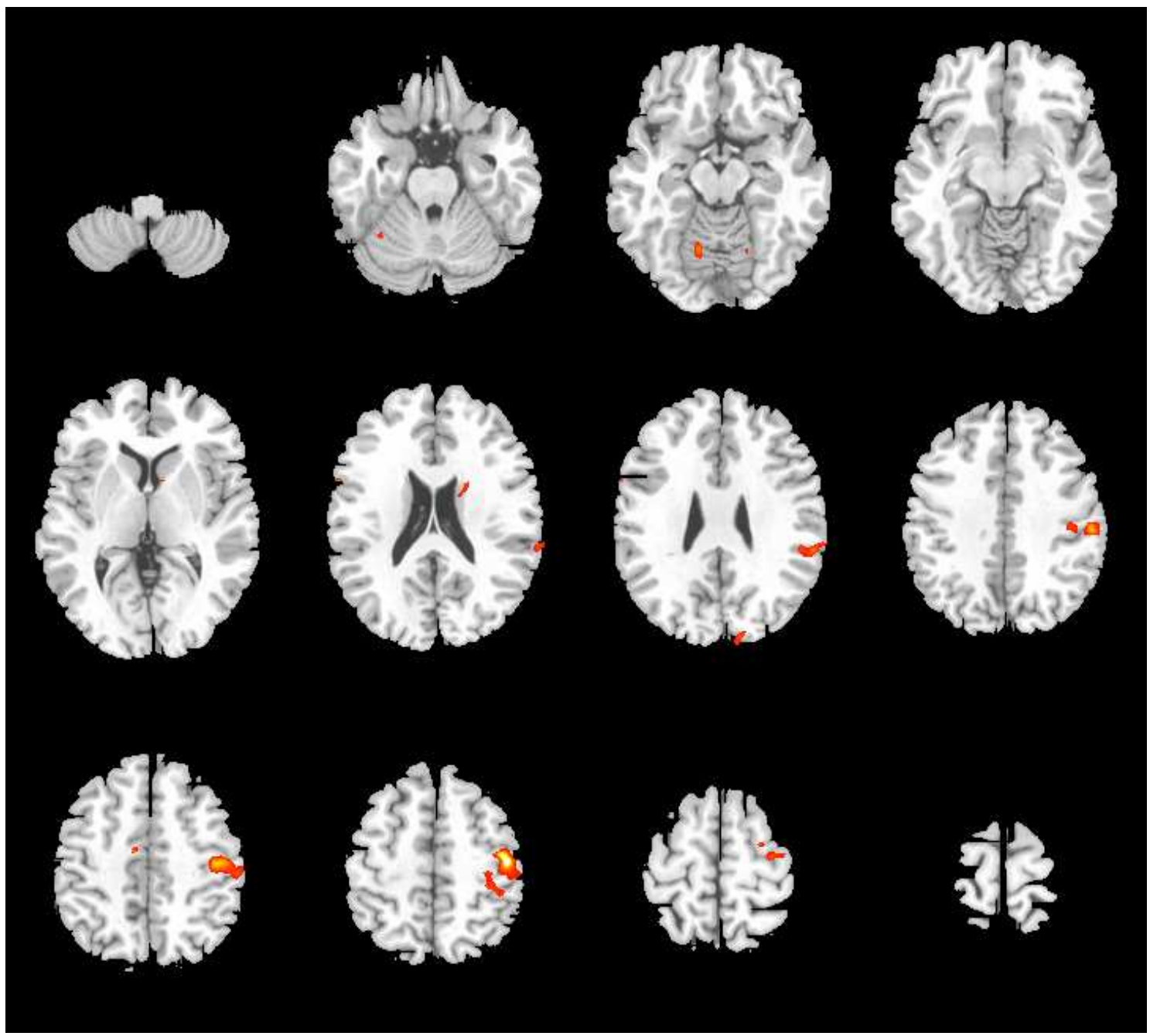

Figura 6.9: Mapa de ativação do grupo normal $(p-$ valor $<0.001)$. 


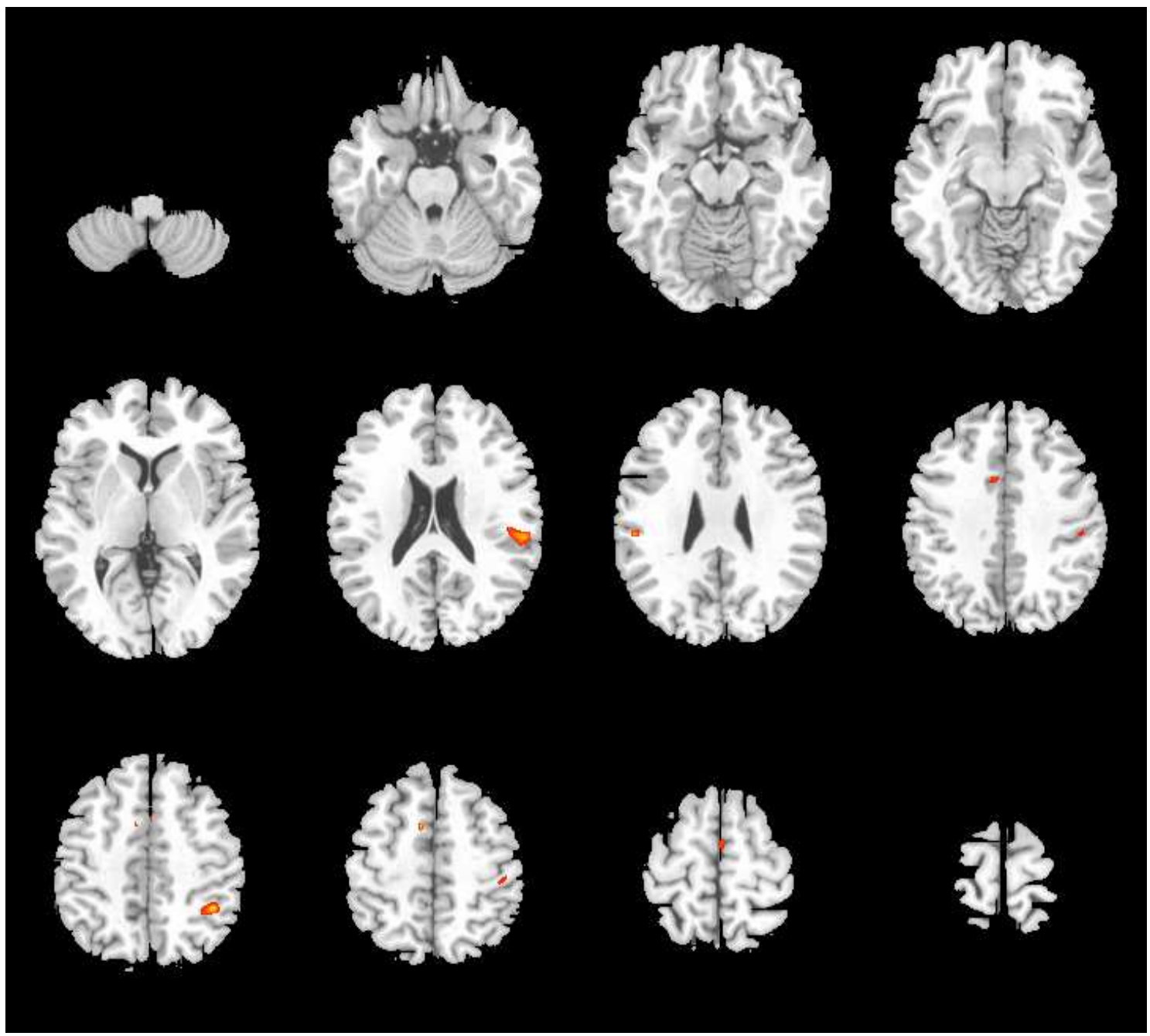

Figura 6.10: Mapa de ativação do grupo de doentes de Parkinson $(p-$ valor $<0.001)$. 


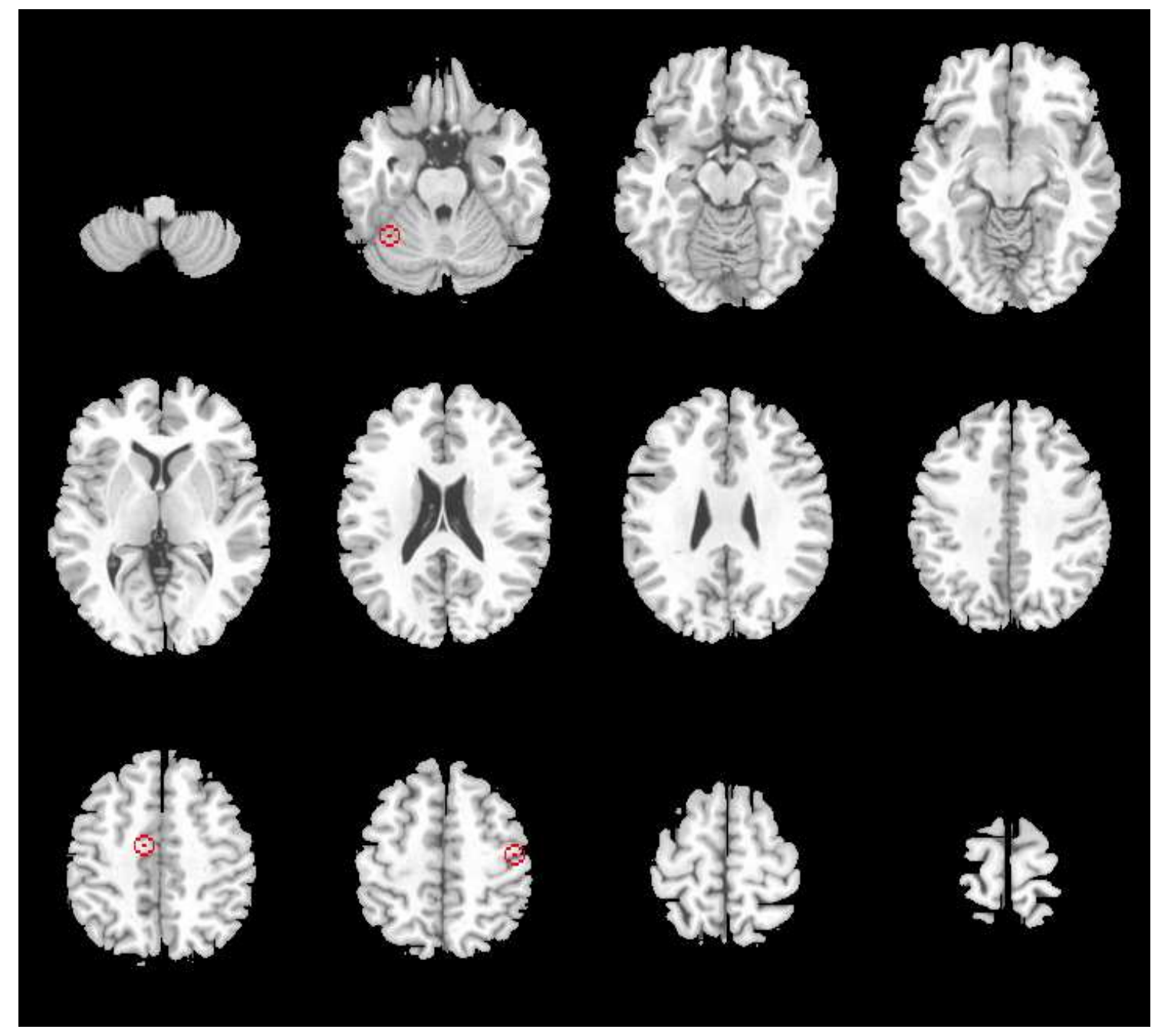

Figura 6.11: Regiões de Interesse: cerebelo direito, área suplementar motora, área motora primária esquerda. 


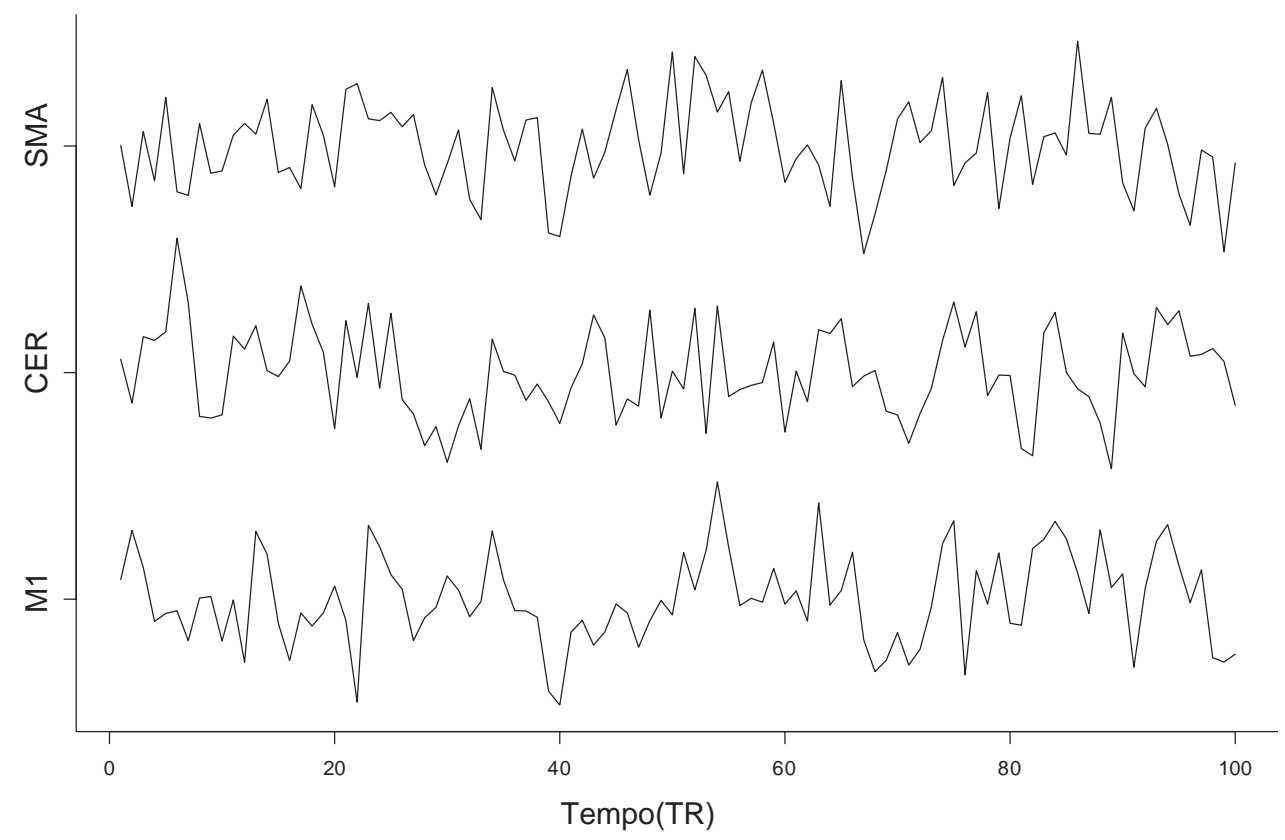

Figura 6.12: Sinal BOLD das áreas de interesse de um indivíduo normal.

regiões são participantes no circuito de programação e execução do movimento. Assim sendo, verifica-se que os resultados obtidos através da análise são neurofisiologicamente plausíveis e trazem novas evidências para uma melhor caracterização da doença.

Enfim, concluímos que os resultados obtidos através do modelo autoregressivo vetorial baseado em ondaletas são razoáveis, e enfatizam alterações de conectividade da rede de programação motora em pacientes com o mal de Parkinson, em acordo com as teorias descritas na literatura.

\subsection{Experimento de Rotação Mental}

As imagens deste estudo foram adquiridas no Institute of Psychiatry - King's College London (Inglaterra) pela psicóloga Christine Ecker, sob orientação do Prof. Michael John Brammer. Utilizou-se um scanner 1.5 Tesla GE Signa Neuro-optimized System (General Eletric, Milwaukee, WI, USA), coletando-se o total de 760 volumes ponderados em $\mathrm{T} 2 *(25$ fatias, espessura $=5 \mathrm{~mm}$, orientação AC-PC, $\mathrm{TE}=40 \mathrm{~ms}, \mathrm{TR}=2000 \mathrm{~ms}$, theta $=80$ 

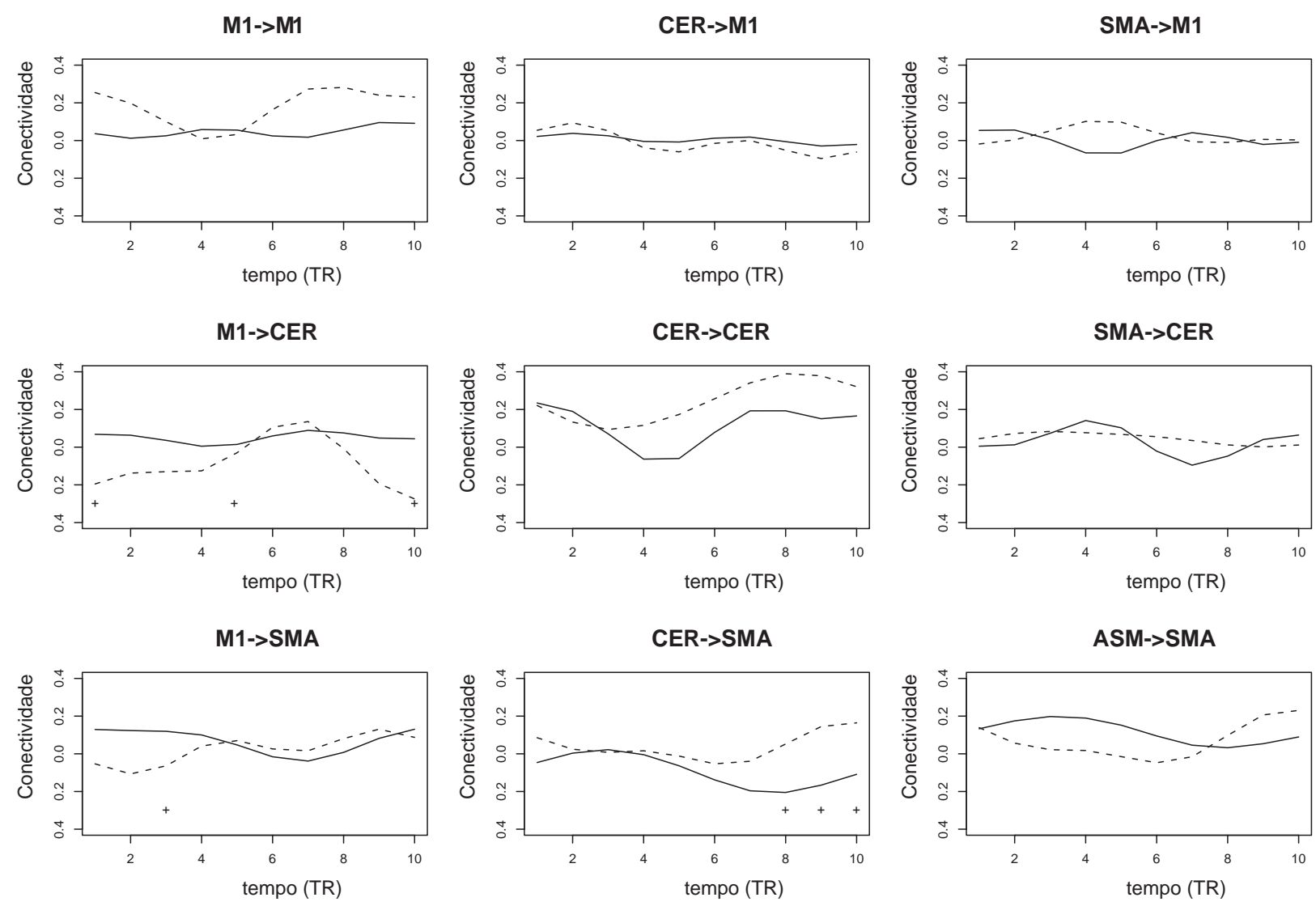

Figura 6.13: Funções de conectividade. As curvas sólidas e tracejadas representam a função de conectividade do grupo normal e doentes de Parkinson, respectivamente. Os marcadores $(+)$ indicam instantes de tempo em que as duas curvas são estatisticamente distintas $(p-$ valor $<0.05)$. 
graus, gap $=0.5 \mathrm{~mm}$, matriz XY de $64 \mathrm{x} 64$ ). Os primeiros e os ultimos 60 volumes foram coletados sob a condição de descanso (resting state), de forma que somente 640 volumes contém informações relacionadas à tarefa de rotação mental.

Onze indivíduos do sexo feminino, destras, com histórico livre de problema neurológicos e psiquiátricos foram voluntárias neste estudo, realizado em acordo com o comitê de ética de Londres e NHS Trust. O experimento consistiu na realização de tarefas de rotação mental, com diferentes níveis de dificuldade (0, 20, 60 e 100 graus de rotação), onde o indivíduo tinha de decidir se dois objetos são idênticos, exceto por uma rotação. Os objetos apresentados eram tridimensionais e similares aos objetos de Shepard-Metzler (1971, vide Figura 6.14A). Assim que os objetos eram apresentados, 8 volumes eram coletados para avaliar a evolução temporal do sinal BOLD. Os níveis de dificuldade foram distribuidos aleatóriamente, com 20 réplicas para cada tipo, resultando na apresentação de 80 estímulos.

Os resultados de áreas ativação deste estudo (Figura 6.14B) foram previamente apresentados em Ecker et al.(2006). No entanto, alguns estudos de neuroimagem (Jordan et al., 2002) apresentaram evidências que tarefas de rotação mental não são processadas num único modulo neural, mas em uma rede distribuída envolvendo áreas visuais associativas e áreas motoras. No entanto, pouco se sabe sobre essa estrutura de conectividade. Assim sendo, o modelo VAR com coeficientes variando no tempo foi aplicado para a modelagem da conectividade, de forma a identificar alteções no fluxo de informação durante a tarefa. Primeiramente, selecionou-se 3 áreas ativadas como regiões de interesse: cortex parietal esquerdo (visual associativo), área pré-suplementar motora medial (tarefas complexas, programação do movimento) e cortex pré-motor esquerdo (programação do movimento).

A rede de conectividade entre as 3 áreas selecionadas de cada indivíduo foi modelada separadamente. O principal interesse deste estudo é a avaliação de diferenças na estrutura de conectividade entre a condição onde não há rotação mental e rotação de 100 graus. Assim sendo, para cada curva de conectividade e para cada ponto no tempo, utilizou-se o teste de Mann-Whitney pareado, comparando-se as curvas de 0 graus com as de 100 graus de rotação. Assim, verifica-se se a diferenças na conectividade entre as duas tarefas é consistente entre os indivíduos. As curvas médias do sinal BOLD para cada área e para cada condição são apresentadas na Figura 6.14C. Os resultados da análise de conectividade são apresentados na Figura 6.15.

O envolvimento de áreas motoras em tarefas de rotação mental é um ponto inte- 

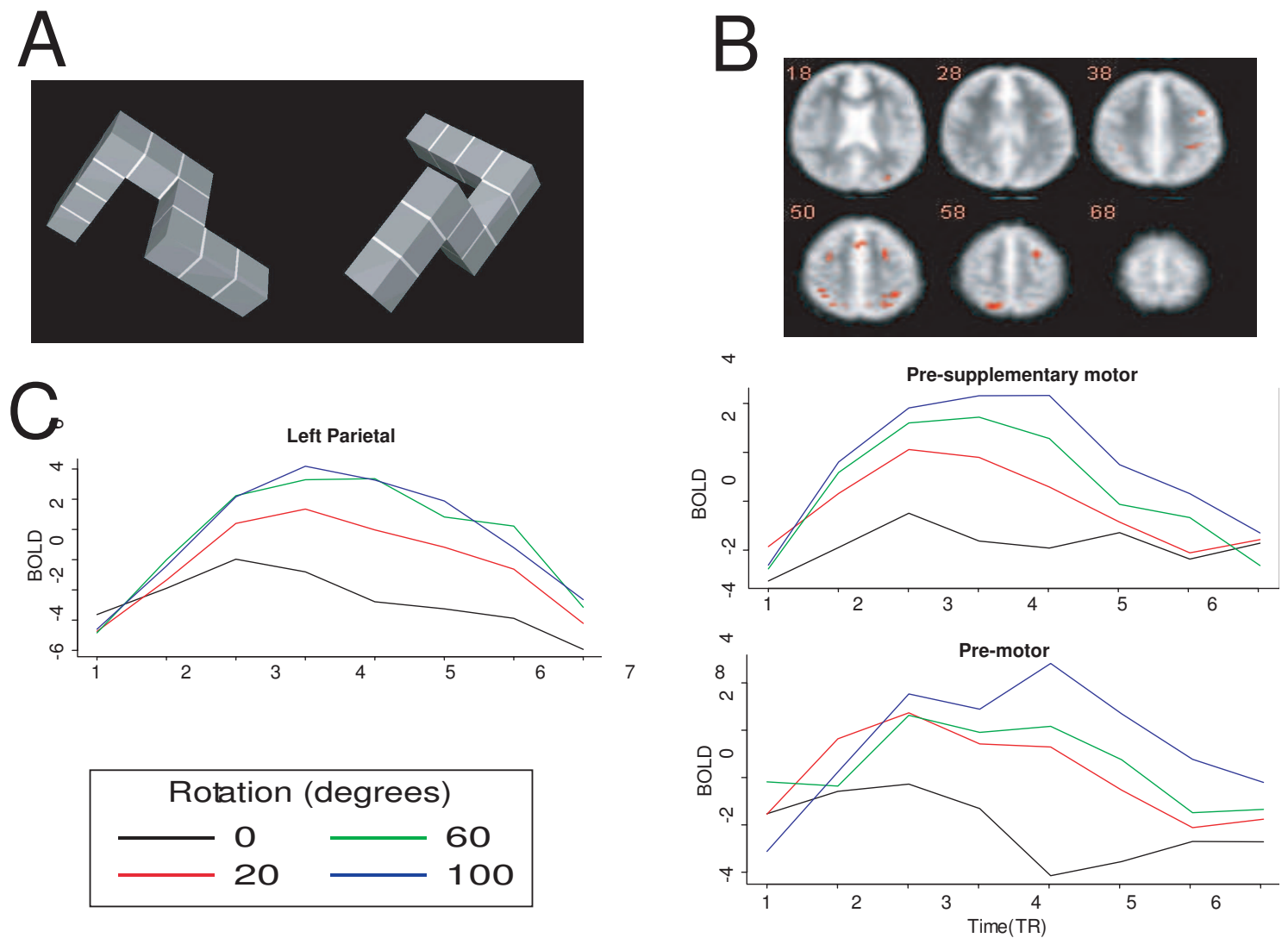

Figura 6.14: A) Objetos tridimensionais utilizados no estudo. B) Mapas de diferenças de ativação entre as condições de 0 e 100 graus de rotação dos objetos $(p-$ valor $<0.001$, corrigido por FDR). C) Sinal BOLD médio das áreas de interesse em diferentes condições (início do estímulo no tempo zero). 

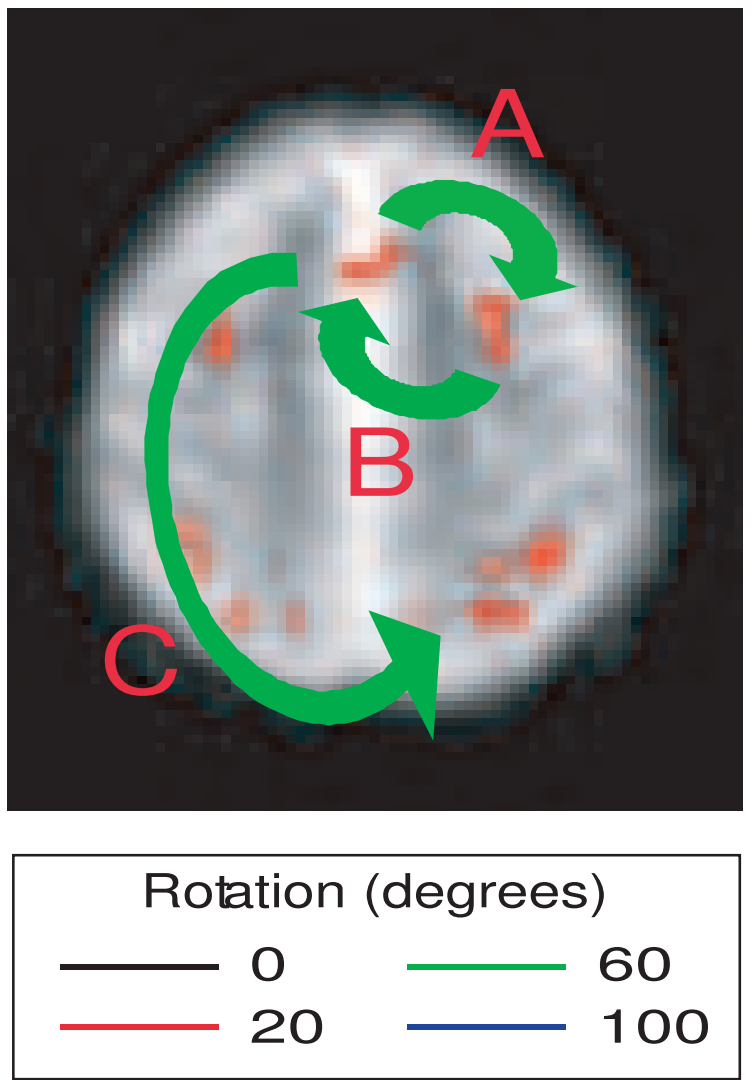

A
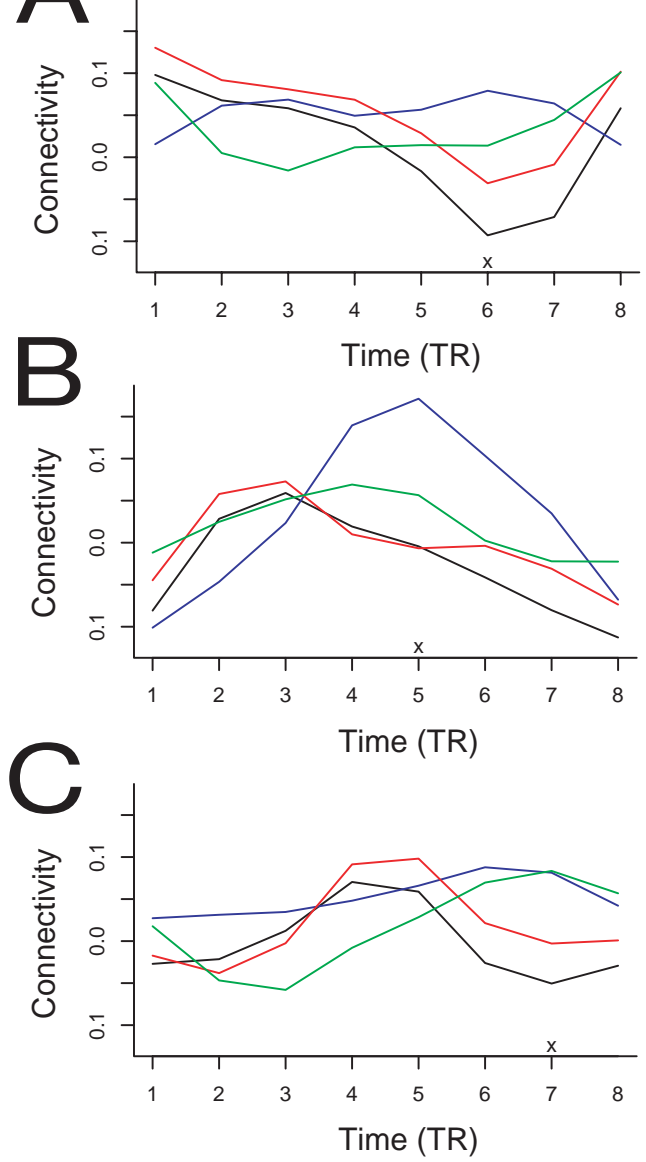

Figura 6.15: Resultado da análise de conectividade. As setas indicam as conectividades que apresentaram diferença na intensidade entre as condições 0 e 100 graus de rotação, para pelo menos um ponto no tempo (p-valor $<0.01)$. O estímulo iniciou-se no tempo zero. 
ressante a ser estudado. Ecker et al.(2006) e Jordan et al. (2002) sugerem que isso se deve ao fato de que para alguns objetos, como os de Shepard-Metzler, indivíduos tendem realizar a tarefa imaginando as próprias mão rotacionando tais objetos. Isso explicaria a existência de alta ativação em áreas pré-motoras e pré-suplementar motoras e não nas áreas motoras primárias (Kosslyn et al, 2001). As áreas pré-motoras são freqüentemente relacionadas à programação e planejamento de movimento, sendo ativadas até mesmo quando indivíduos imaginam o movimento. Tal fato corrobora a hipótese de que a rotação mental e visualização de movimentos estão relacionados. A área pré-suplementar motora (cortex pré-frontal) está, na realidade, relacionada com a realização de diversas tarefas complexas, tais como associações visuo-motoras (Sakai et al., 1999) e atualizações do planejamento motor (Shima et al., 1996) . A análise de conectividade usando o modelo proposto sugere que hipótese de interação entre a área visual associativa e áreas de planejamento motor em tarefas de rotação mental é válida. É interessante verificar que nos pontos no tempo em que a curva de conectividade de 0 graus é diferente de 100 graus, existe uma variação gradual para os outros ângulos, enfatizando a consistência do resultado.

Assim, como há diferenças na conectividade entre as áreas selecionadas durante o processo de rotação mental, conclui-se que tais conexões estão relacionadas à tarefa. 


\section{CAPÍtulo 7}

\section{Conclusão}

A utilização de experimentos com a aquisição de imagens via ressonância magnética funcional vêm crescendo rapidamente. A grande maioria dos estudos encontrados na literatura focam apenas a identificação de áreas ativadas durante a realização de certos experimentos. No entanto, uma das características mais fundamentais da neurofisiologia cerebral é o processamento paralelo e interação entre módulos neuronais. Assim, a utilização de modelo multivariados para a identificação de redes neuronais e estruturas de conectividade é essencial para um melhor entendimento das dinâmicas e mecanismos cerebrais.

Como o cérebro apresenta um caráter dinâmico, isto é, nível de atividade e fluxo de informação variante no tempo, a utilização de métodos capazes de identificar e modelar processos dessa natureza se faz desejável. Neste trabalho, introduzimos os modelos autoregressivos multivariados com coeficientes variando no tempo, como uma possível metodologia de análise. As propriedades estatísticas e assintóticas foram exploradas e resultados empíricos através de simulações computacionais evidenciam a aplicabilidade do modelo.

Além disso, algumas aplicações do modelo e metodologias propostas sobre dados reais de ressonância magnética funcional são apresentadas, ilustrando possíveis casos onde método pode contribuir com novas informações e descobertas.

Projetos futuros envolvem o estabelecimento de medidas de diagnóstico de ajuste e critérios de seleção de modelos. A qualidade de ajuste pode ser verificada explorando-se propriedades dos resíduos. A utilização de critérios como AIC, BIC, MDL ou procedimentos de validação cruzada (cross - validation) são fortes candidatos para a escolha da 
ordem do modelo autoregressivo, bem como a escala de truncamento da expansão wavelet. Por fim, novas aplicações do modelo proposto nas áreas de bioinformática e séries temporais financeiras estão começando a ser exploradas. 


\section{Referências Bibliográficas}

Abler B., Roebroeck A., Goebel R., Hose A., Schonfeldt-Lecuona C., Hole G., Walter H. (2006). Investigating directed influences between activated brain areas in a motorresponse task using fMRI. Magn Reson Imaging, 24(2):181-5.

Amaro Jr. E. and Yamashita H. (2001). Aspectos básicos de tomografia computadorizada e ressonância magnética. Rev Bras Psiquiatr, 23 (Supl.I):2-3

Amaro Jr. Ressonância magnética funcional relacionada a eventos. (2000). Rev Psiq Clín, 27-33

Andrews D.W. (1988). Laws of large number for dependent non-identically distributed random variables. Econometric Theory, 4:458-67.

Baccalá L.A. and Sameshima K. (2001). Partial directed coherence: a new concept in neural structure determination. Biological Cybernetics. 8:463-74.

Bandettini P.A., Jesmanowicz A., Wong E.C. and Hyde J.S.(1993). Processing strategies for time-course data sets in functional MRI of the brain. Magn Reson Med, 30:161-173.

Biswal B., Yetking F.Z., Haughton V.M., Hyde J.S. (1995). Functional connectivity in the motor cortex of resting human brain using echo-planar MRI. Magn Reson Med 34:537-41. 
Brammer, M. J., E. T. Bullmore, Simmons A., Williams S.C., Grasby P.M., Howard R.J., Woodruff P.W., Rabe-Hesketh S. (1997). Generic brain activation mapping in functional magnetic resonance imaging: a nonparametric approach. Magn Reson Imaging 15(7):763-70.

Büchel C. and Friston K. (1997). Modulation of connectivity in visual pathways by attention: Cortical interactions evaluated with structural equation modeling and fMRI. Cerebral Cortex. 7:768-78.

Bullmore E.T., Brammer M.J. and Williams S.C.R., Rabe-Hesketh S., Janot N., David A., Mellers J., Howard R., Sham P. (1996). Statistical methods of estimation and inference for functional MR image analysis. Magn Reson in Med, 35, 261-77.

Bullmore E.T., Long C., Suckling J., Fadili J., Calvert G., Zelaya F., Carpenter T.A., Brammer M.J. (2001). Colored noise and computational inference in neurophysiological (fMRI) time series analysis: resampling methods in time and wavelet domains. Hum Brain Mapp, 12:61-78.

Chiann C. and Morettin P.A. (1998). A wavelet analysis for time series. J Non-Par Stat, 10:1-46.

Coull J. T., Frith C. D. , Buchel C., Nobre A.C. (2000). Orienting attention in time: behavioural and neuroanatomical distinction between exogenous and endogenous shifts. Neuropsychologia, 38(6):808-19.

Cui S. Z., Li E. Z., Zang Y.F., Weng X.C., Ivry R., Wang J.J. (2000). Both sides of human cerebellum involved in preparation and execution of sequential movements. Neuroreport, 11(17):3849-53.

Cunnington R., Windischberger C., Deecke L., Moser E. (2002). The preparation and execution of self-initiated and externally-triggered movement: a study of event-related fMRI. Neuroimage, 15(2):373-85. 
D'Esposito M., Ballard D., Zarahn E., Aguirre G.K. (2000). The role of prefrontal cortex in sensory memory and motor preparation: an event-related fMRI study. Neuroimage, $11: 400-8$

Dahlhaus R. (2000). A likelihood approximation for locally stationary processes. The Annals of Statistics, 28:1762-94.

Dahlhaus R., Eichler M., Sandkuhler J. (1999). Identification of synaptic connections in neural ensembles by graphical models. J Neurosci Methods, 77(1):93-107.

Dahlhaus R., Neumann M.H. and von Sachs R. (1999). Nonlinear wavelet estimation of time-varying autoregressive processes. Bernoulli, 5:873-906.

Daubechies I. (1992). Ten Lectures on Wavelets. Philadelphia. SIAM.

DeLong M.R. (1990). Primate models of movement disorders of basal ganglia origin. Trends Neurosci, 13(7):281-5.

Donoho D.L. and Johnstone I. M. (1994). Ideal spatial adaptation by wavelet shrinkage. Biometrika, 81:425-55.

Donoho D.L. and Johnstone I. M. (1995a). Adapting to unknown smoothing via wavelet shrinkage. JASA, 90:1200-24.

Donoho D.L. and Johnstone I. M. (1998). Minimax estimation via wavelet shrinkage. Annals of Statistics, 26 3:879-921.

Donoho D.L., Johnstone, I.M., Kerkyacharian, G. and Picard, D. (1995b). Wavelet shrinkage: asymptopia? (with discussion). JRSS B, 57:301-69.

Durbin J. (1959). Estimation of parameters in time series regression models. JRSS B, 22:139-53. 
Ecker C., Brammer M.J., David A.S., Williams S.C. (2006). Time-resolved fMRI of mental rotation revisited-dissociating visual perception from mental rotation in female subjects. Neuroimage, 32(1):432-44.

Efron B. and Tibshirani R.J. (1993). An introduction to the bootstrap. New York, Chapman and Hall.

Eichler M. (2005). A graphical approach for evaluating effective connectivity in neural systems. Philos Trans $R$ Soc Lond B Biol Sci, 360(1457):953-67.

Frackowiak R.S.J.,Frith C., Dolan R., Price C.J., Zeki S., Ashburner J. and Penny W.D. (2003). Human Brain Function. 2nd ed. Academic Press.

Friston K.J., Harrison L., Penny W. (2003). Dynamic causal modelling. Neuroimage, 19(4):1273-302.

Friston K.J., Worsley K.J., Frakowiak R.S.J., Mazziotta J.C. and Evans A.C. (1994). Assessing the significance of focal activations using their spatial extent. Human Brain Mapping, 1:210-20.

Friston K.J., Ashburner J.T., Kiebel S.J., Nichols T.E., Penny W.D. (2007). Statistical Parametric Mapping: the analysis of functional brain images. Elsevier, London.

Goebel R., Roebroeck A., Kim D.S., Formisano E. (2003). Investigating directed cortical interactions in time-resolved fMRI data using vector autoregressive modeling and Granger causality mapping. Magn Resson Imag, 21:1251-61.

Granger C.W.J. (1969). Investigating causal relations by econometric models and crossspectral methods. Econometrica, 37:424-38.

Graybill F. (1985). Theory and Application of the Linear Model. Wadsworth Publishing Company, Inc., Belmont, California. 
Grossmann A. and Morlet J. (1984). Decomposition of Hardy functions into square integrable wavelets of constant shape. SIAM J Math, 15:723-36.

Grossmann A. and Morlet J. (1985). Decomposition of Functions into wavelets of constant shape, and related transforms. In: STREIT, L. (ed.). Mathematics + Physics, Lectures on Recent Results. Singapore, World Scientific, 135-65.

Hall D. A., Haggard M.P., Akeroyd M.A., Summerfield A.Q., Palmer A.R., Elliott M.R., Bowtell R.W. (2000). Modulation and task effects in auditory processing measured using fMRI. Hum Brain Mapp, 10(3): 107-19.

Hamilton J.D. (1994). Time Series Analysis. Princeton U. Press, Princeton.

Hampson M., Peterson B.S., Skudlarski P., Gatenby J.C., Gore J.C. (2002). Detection of functional connectivity using temporal correlations in MR images. Hum Brain Mapp, $15: 247-62$.

Harrison L., Penny W.D., Friston K. (2003). Multivariate autoregressive modeling of fMRI time series. Neuroimage, 19(4):1477-91.

Ito K., Morrish P.K., Rakshi J.S., Uema T., Ashburner J., Bailey D.L., Friston K.J., Brooks D.J. (1999). Statistical Parametric Mapping with 18F-dopa PET shows bilaterally reduced striatal and nigral dopaminergic function in early Parkinson's disease. Journal Neurol Neurosurg Psychiatry, 66(6):754-8.

Jack Jr. C.R., Lexa F.J., Traojanowski J.Q., Braffman B.H., Atlas S.W. (2002). Normal aging, dementia. and degenerative disease. Magn Reson of the Brain and Spine, 1177:240.

Jancke L., Himmelbach M., Shah N.J, Zilles K. (2000). "The effect of switching between sequential and repetitive movements on cortical activation." Neuroimage, 12(5):528-37.

Jordan K., Wustenberg T., Heinze H.J., Peters M., Jancke L. (2002). Women and men exhibit different cortical activation patterns during mental rotation tasks. Neuropsycho- 
logia, 40:2397-408.

Kermadi I., Liu Y., Rouiller E.M. (2000). Do bimanual motor actions involve the dorsal premotor (PMd), cingulate (CMA) and posterior parietal (PPC) cortices? Comparison with primary and supplementary motor cortical areas. Somatosens Mot Res, 17(3):255-71.

Kosslyn S.M., Thompson W.L., Wraga M., Alpert N.M. (2001). Imagining rotation by endogenous versus exogenous forces: distinct neural mechanisms. NeuroReport, 12:2519-525.

Kurata K., Tsuji T., Naraki S., Seino M., Abe Y. (2000). Activation of the dorsal premotor cortex and pre-supplementary motor area of humans during an auditory conditional motor task. J Neurophysiol, 84(3):1667-72.

Lange N. and Zeger S.L. (1997). Non-linear Fourier time series analysis for human brain mapping by functional magnetic resonance imaging. JRSS A, 46:1-29.

Lee K. M., Chang K.H., Roh J.K. (1999). Subregions within the supplementary motor area activated at different stages of movement preparation and execution. Neuroimage, $9(1): 117-23$.

Logothetis N.K. (2003). MR imaging in the non-human primate: studies of function and of dynamic connectivity. Current Opinion in Neurobiol, 13:630-42.

Luppino G., Matelli M., Camarda R., Rizzolatti G. (1993). Corticocortical connections of area F3 (SMA-proper) and area F6 (pre-SMA) in the macaque monkey. J Comp Neurol, 338(1):114-40.

Lütkepohl H.(1993). Introduction to multiple time series analysis. Springer-Verlag. New York.

Lutz K., Specht K., Shah N.J., Jancke L. (2000). Tapping movements according to regular and irregular visual timing signals investigated with fMRI. Neuroreport, 11(6):1301-6. 
Mallat S. (1989). A theory for multiresolution signal decomposition: wavelet representation. IEEE Trans Pattern Anal Mach Intellig, 11:674-93.

Matelli M., Luppino G., Rizzolatti G. (1991). Architecture of superior and mesial area 6 and the adjacent cingulate cortex in the macaque monkey. J Comp Neurol, 311(4):445-62.

McIntosh A.R.(1999). Mapping cognition to the brain through neural interactions. Memory, 7(5-6):523-48.

Nasser J.A., Falavigna A., Alaminos A., Bonatelli, A., Ferraz F. (2002). Deep brain stimulation of subthalamic nucleous in Parkinson's disease. Arq Neuropsiquiatr, 60(1):86-90.

Ogawa S., Lee T.M., Kay A.R., Tank D.W. (1990). Brain magnetic resonance imaging with contrast dependent on blood oxygenation. Proc Natl Acad Sci USA, 87(24):9868-72

Ohara S., Mima T., Baba K., Ikeda A., Kunieda T., Matsumoto R., Yamamoto J., Matsuhashi M., Nagamine T., Hirasawa K., Hori T., Mihara T., Hashimoto N., Salenius S., Shibasaki H. (2001). "Increased synchronization of cortical oscillatory activities between human supplementary motor and primary sensorimotor areas during voluntary movements." J Neurosci, 21(23):9377-86.

Raichle M.E., MacLeod A.M., Snyder A.Z., Powers W.J., Gusnard D.A., Shulman G.L. (2001). A default mode of brain function. Proc Natl Acad Sci U S A, 98(2):676-82.

Rizzolatti G., Luppino G., Matelli M. (1996). The classic supplementary motor area is formed by two independent areas. Adv Neurol, 70:45-56.

Rushworth M.F., Walton M.E., Kennerley S.W., Bannerman D.M. (2004). Action sets and decisions in the medial frontal cortex. Trends Cogn Sci, 8(9):410-7.

Sakai K., Hikosaka O., Miyauchi S., Sasaki Y., Fujimaki N., Putz B. (1999). Presupplementary motor area activation during sequence learning reflects visuo-motor association. J Neurosci, 19, RC1.t. 
Sato J.R., Amaro Jr E., Takahashi D.Y., Felix M.M., Brammer M.J., Morettin P.A. (2006a). A method to produce evolving functional connectivity maps during the course of an fMRI experiment using wavelet-based time-varying Granger causality. Neuroimage, $31: 187-96$.

Sato J.R., Takahashi D.Y., Cardoso E.F., Morais-Martin M.G., Amaro Jr E., Morettin P.A. (2006b). Intervention models in functional connectivity identification applied to fMRI. Int $J$ of Biomed Imag, 1:1-7.

Sen P.K. and Singer J.M. (1993). Large Sample Methods in Statistics: An Introduction with Applications. New York: Chapman and Hall.

Shepard RN and Metzler J (1971). Mental rotation of three-dimensional objects. Science, 171:701-703.

Shima K., Mushiake H., Saito N., Tanji J. (1996). Role for cells in the presupplementary motor area in updating motor plans. Proc Nat Acad Sci USA, 93:8694-698.

Sobotta J. (1993). Atlas of Human Anatomy, 12th Ed., Vol 2. Urban and Schwarzenberg.

Stephan K.M., Binkofski F., Halsband U., Dohle C., Wunderlich G., Schnitzler A., Tass P., Posse S., Herzog H., Sturm V., Zilles K., Seitz R.J., Freund H.J. (1999). The role of ventral medial wall motor areas in bimanual co-ordination. A combined lesion and activation study. Brain, 122:351-68.

Talairach J. and Tournoux P. (1988). Co-planar stereotaxic atlas of the human brain: 3-dimensional proportional system: an approach to cerebral imaging. Stuttgart: Thieme.

Toni I., Thoenissen D., Zilles K. (2001). Movement preparation and motor intention. Neuroimage, 14:110-7.

Thulborn K.R., Waterton J.C., Mathews P.M., Radda G.K. (1982). Oxygenation de- 
pendence of the transverse relaxation time of water protons in whole blood at high field. Bioch Bioph Acta, 714:265-70.

Valdes-Sosa P.A. (2004). Spatio-temporal autoregressive models defined over brain manifolds. Neuroinformatics, 2(2):239-50.

Valdes-Sosa P.A., Sanchez-Bornot J.M., Lage-Castellanos A., Vega-Hernandez M., BoschBayard J., Melie-Garcia L., Canales-Rodriguez E. (2005). Estimating brain functional connectivity with sparse multivariate autoregression. Philos Trans $R$ Soc Lond B Biol Sci, 360(1457):969-81.

Viallet F., Massion J., Massarino R., Khalil R. (1992). Coordination between posture and movement in a bimanual load lifting task: putative role of a medial frontal region including the supplementary motor area. Exp Brain Res, 88(3):674-84.

Vidakovic B. (1999). ÈStatistical Modeling by WaveletsÉ. Wiley Series in Probability and Statistics.

von Sachs R., Nason G.P., Kroisandt G. (1996). Spectral representation and estimation for locally stationary wavelets processes. FB Mathematik, Kaiserslautern University, D67653, Kaiserslautern, Germany.

von Sachs R., Nason G.P., Kroisandt G. (1997). Adaptative estimation of the evolutionary wavelet spectrum. Technical Report 516, Department of Statistics. Stanford University.

Wenderoth N., Debaere F., Sunaert S., Swinnen S.P. (2005). Spatial interference during bimanual coordination: Differential brain networks associated with control of movement amplitude and direction. Hum Brain Mapp, 26(4):286-300.

Wenderoth N., Debaere F., Sunaert S., Swinnen S.P. (2005). The role of anterior cingulate cortex and precuneus in the coordination of motor behaviour. Eur $J$ Neurosci, $22(1): 235-46$. 
Wenderoth N.,Debaere F., Sunaert S., van Hecke P., Swinnen S.P. (2004). Parietopremotor areas mediate directional interference during bimanual movements. Cereb Cortex, 14(10):1153-63.

White H. (1984). Asymptotic Theory for Econometricians. New York: Academic Press.

Worsley K.J. and Friston K.J. (1995). Analysis of fMRI time series revisited - again. NeuroImage, 2:173-81. 